\title{
Global Distribution, Public Health and Clinical Impact of the Protozoan Pathogen Cryptosporidium
}

\author{
Lorenza Putignani and Donato Menichella \\ Microbiology Unit, Bambino Gesù Pediatric Hospital, Scientific Institute, Piazza Sant'Onofrio 4, 00165 Rome, Italy \\ Correspondence should be addressed to Lorenza Putignani, 1_putignani@yahoo.com
}

Received 4 September 2009; Revised 7 January 2010; Accepted 11 May 2010

Academic Editor: Bettina Fries

Copyright ( $) 2010$ L. Putignani and D. Menichella. This is an open access article distributed under the Creative Commons Attribution License, which permits unrestricted use, distribution, and reproduction in any medium, provided the original work is properly cited.

Cryptosporidium spp. are coccidians, oocysts-forming apicomplexan protozoa, which complete their life cycle both in humans and animals, through zoonotic and anthroponotic transmission, causing cryptosporidiosis. The global burden of this disease is still underascertained, due to a conundrum transmission modality, only partially unveiled, and on a plethora of detection systems still inadequate or only partially applied for worldwide surveillance. In children, cryptosporidiosis encumber is even less recorded and often misidentified due to physiological reasons such as early-age unpaired immunological response. Furthermore, malnutrition in underdeveloped countries or clinical underestimation of protozoan etiology in developed countries contribute to the underestimation of the worldwide burden. Principal key indicators of the parasite distribution were associated to environmental (e.g., geographic and temporal clusters, etc.) and host determinants of the infection (e.g., age, immunological status, travels, community behaviours). The distribution was geographically mapped to provide an updated picture of the global parasite ecosystems. The present paper aims to provide, by a critical analysis of existing literature, a link between observational epidemiological records and new insights on public health, and diagnostic and clinical impact of cryptosporidiosis.

\section{Introduction}

1.1. The Cryptosporidium Parasite: General Description. Infections of the human gastrointestinal tract with enteric pathogens are among the leading causes of disease, suffering, and death worldwide. Enteric pathogens are ingested with contaminated water and food and pass through the entire gastrointestinal tract. After establishment in a host, the infection spread to new hosts by a subsequent shedding. The most important and prevalent infections of the small intestine are caused by diarrheagenic Escherichia coli, particularly enterotoxigenic and enteropathogenic E. coli, Rotavirus, Giardia lamblia, and Cryptosporidium parvum [1-3]. Particularly, more than 58 million cases of diarrhea detected per year in children are associated to intestinal protozoa infections with high morbidity and mortality infection rates [4]. Cryptosporidium spp. are oocysts-forming apicomplexan protozoa. Following ingestion, the oocyst excystation, releases sporozoites which invade enterocytes. The excysted parasites undergo asexual (merogony) and sexual multiplication (gametogony) producing macrogametocytes and microgametocytes. Upon fertilization of the macrogametocytes by microgametes a zygotes is developed which sporulates (sporogony), generating thin-walled oocysts, involved in autoinfection and thick-walled oocysts excreted from the host (Figure 1). Once released in the environment, the parasite may cause enteric infection (cryptosporidiosis) both in humans and animals, mainly transmitted via the fecal-oral route through a zoonotic or anthroponotic modality or via contaminated water or food (Figure 2). In humans the disease results in sickness and severe diarrhea and can be life threatening in the very young, elderly and in immunosuppressed individuals, particularly those with HIV infection [5]. Contamination of drinking water by Cryptosporidium can result in major waterborne outbreaks of cryptosporidiosis [6]; additionally the Cryptosporidium is now increasingly considered an important foodborne pathogen $[7,8]$ causing a disease of socioeconomic significance worldwide. Three features of Cryptosporidium spp. ensure a high level of environmental contamination and 


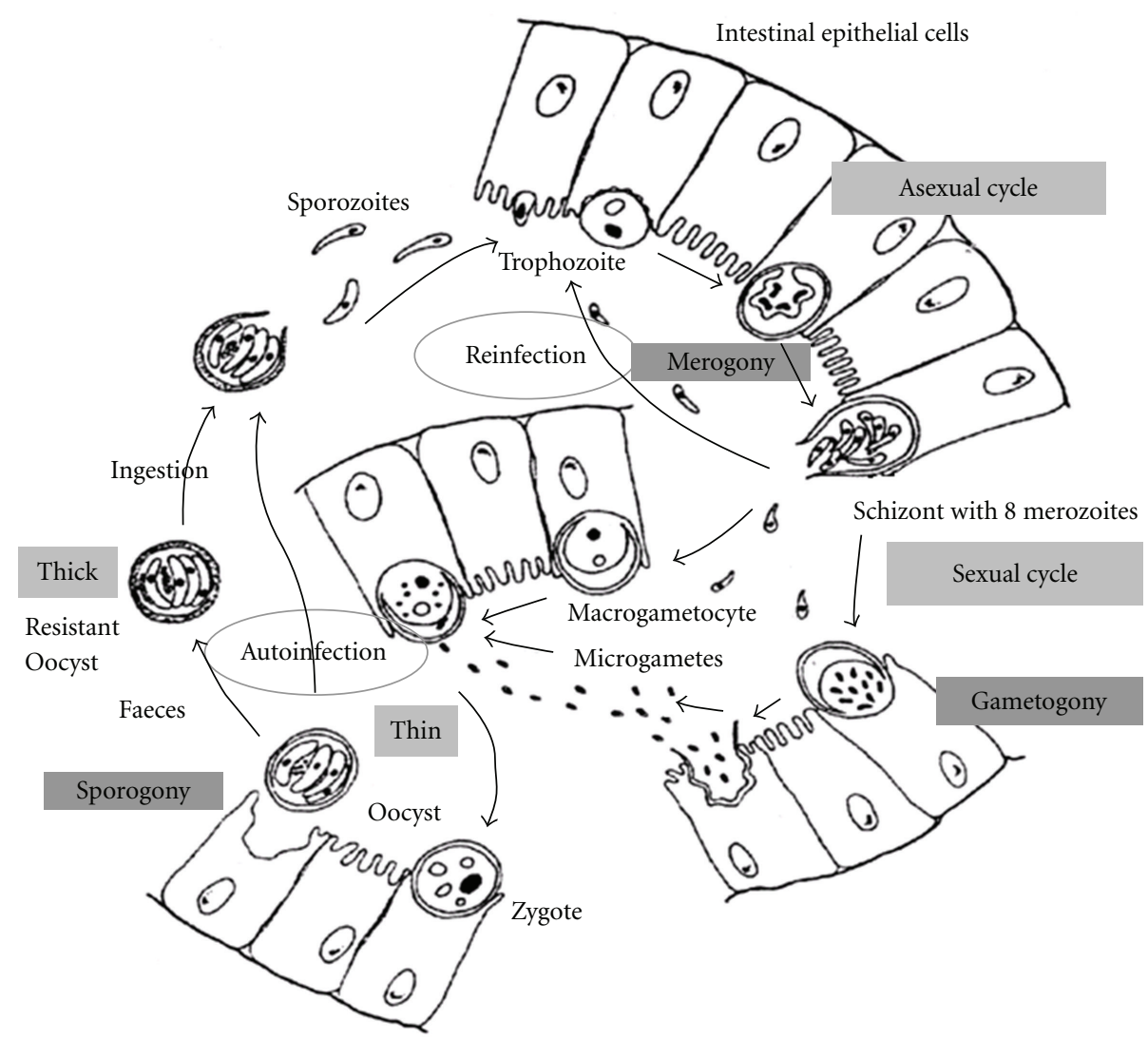

Figure 1: Life cycle of Cryptosporidium in the enterocyte. Following oocyst ingestion by a host, and excystation, the sporozoites are released and parasitize epithelial cells of the gastrointestinal tract. In these cells, the parasites undergo asexual multiplication (schizogony or merogony) and then sexual multiplication (gametogony), producing microgamonts and macrogamonts. Upon fertilization of the macrogamonts by the microgametes, oocysts develop and sporulate in the infected host. Two different types of oocysts are produced: the thick-walled, which is commonly excreted by the host, after sporogony, and the thin-walled oocyst, which is primarily involved in autoinfection. Putignani and Menchella, 2010.

increase the likelihood of waterborne transmission. Firstly, they are responsible for disease in a broad range of hosts including man $[9,10]$, have a low-infectious dose $(10-$ 30 oocysts) enhancing the possibility of infection also in healthy immunocompetent people $[11,12]$, which may shed $10^{8}-10^{9}$ oocysts in a single bowel movement and excrete oocysts for up to 50 days after cessation of diarrhea [13, 14]; secondly, their transmissive stages (oocysts) are small in size and environmentally robust $[15,16]$ and thirdly, they are insensitive to the normal disinfectants commonly used in the water industry $[17,18]$.

\subsection{Cryptosporidium Species and Human Infection. Since the} genus Cryptosporidium was established for Cryptosporidium muris by Tyzzer in 1907, 37 species names have been introduced. However, after redescription and confirmation, currently 21 names are associated with individual species [19] and 16 species are actually regarded as valid on the basis of different oocyst morphology, site of infection, vertebrate class specificity, and genetic differences: $C$. muris in rodents; Cryptosporidium andersoni and Cryptosporidium bovis in cattle and sheep; Cryptosporidium suis in pigs; C. parvum in cattle, humans, and other mammals; Cryptosporidium meleagridis in birds and humans; Cryptosporidium hominis in humans; Cryptosporidium baileyi and Cryptosporidium galli in birds; Cryptosporidium serpentis and Cryptosporidium saurophilum in snakes and lizards; Cryptosporidium molnari and Cryptosporidium scophthalmi in fish; Cryptosporidium wrairi in guinea pigs; Cryptosporidium felis in cats; Cryptosporidium canis in dogs [20]. The majority of these have a dominant host, but they are accidentally found in possibly unusual hosts. Remarkably, Cryptosporidium parasites are not related to other coccidians and the major recognised species in Cryptosporidium separate into two broad groups, with $C$. muris and $C$. serpentis forming one group and C. parvum, C. felis, C. wrairi, C. meleagridis, and C. baileyi forming a second broad group [21]. The accurate identification and characterisation of Cryptosporidium species and population variants are now central in the new taxonomic classification of Cryptosporidium species and in the categorization of genotypes or subtypes [20]. The picture that is emerging as a result of molecular studies clearly indicates that the species level taxonomy of the genus does not reflects the current molecular phylogenetic analyses or epidemiological data, which show high inter- and 


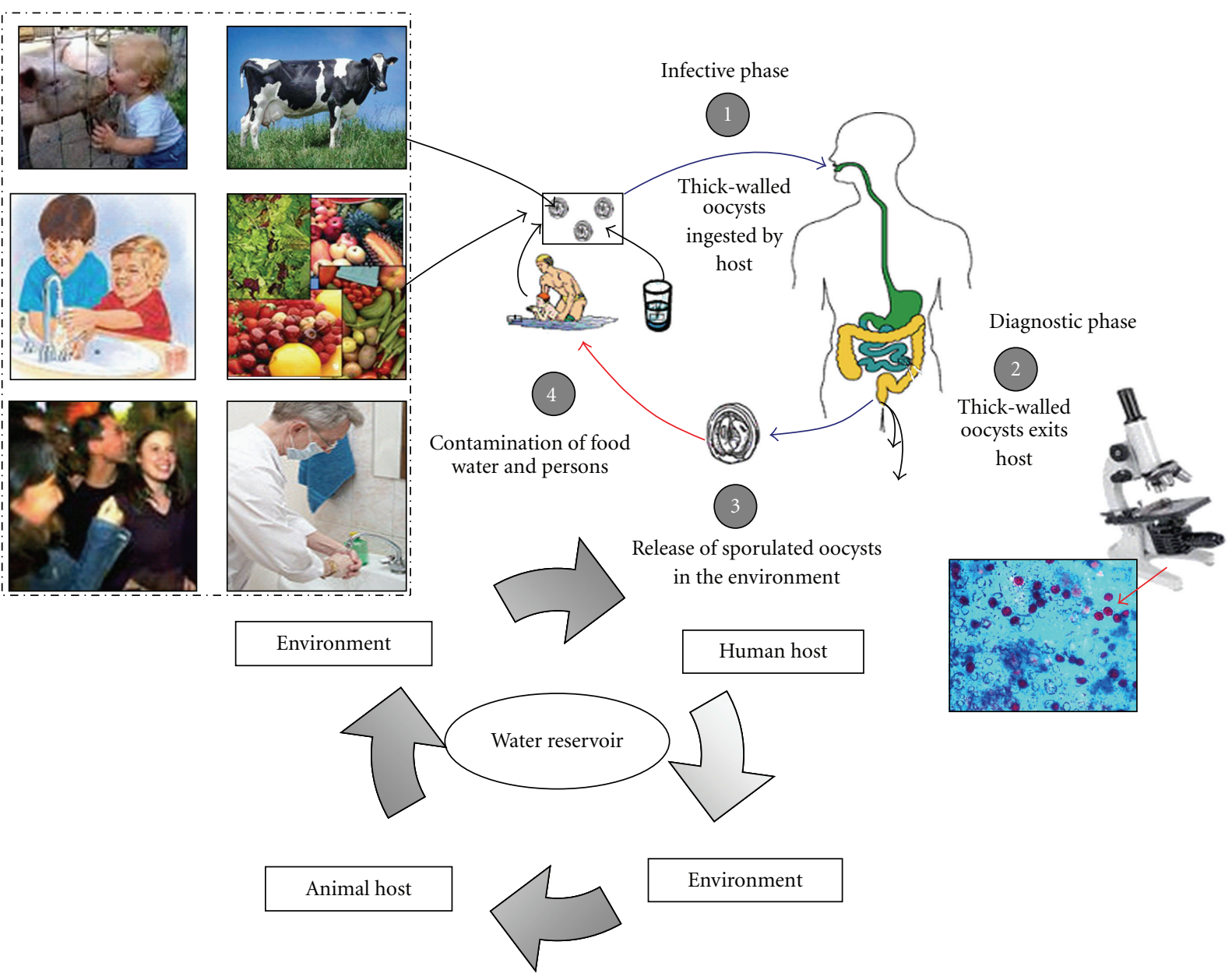

FIGURE 2: Description of transmission modes of Cryptosporidium. Following ingestion (and possibly inhalation) by a suitable host (e.g., human host), excystation occurs (infective stage, (1)). The released sporozoites invade epithelial cells of the gastrointestinal tract or other tissues, complete their cycle producing oocysts which exit host (diagnostic stage, (2)) and are released in the environment (3). Transmission of Cryptosporidium mainly occurs by ingestion of contaminated water (e.g., surface, drinking or recreational water), food sources (e.g., chicken salad, fruits, vegetables) or by person-to-person contact (community and hospital infections) (4). Zoonotic transmission of C. parvum occurs through exposure to infected animals (person-to-animal contact) or exposure to water (reservoir) contaminated by feces of infected animals (4). Putignani and Menchella, 2010.

intraspecific variation, and warrants reappraisal [21]. The vast majority of human cases of cryptosporidiosis worldwide are mainly caused by two species, C. parvum and C. hominis [21]. However other species, including C. felis [22, 23], C. meleagridis $[23,24]$, C. canis $[23,25]$, C. suis [23], C. muris [26], and more rarely C. baileyi [27] can infect humans too, especially children under the age of 5 years and immunocompromised individuals [28]. All Cryptosporidium species are transmitted in the various hosts by ingestion and inhalation of oocysts, irrespective of the species types. However, the clinical and epidemiological significance of various Cryptosporidium species and subtypes in humans is not yet clear. Results of recent genotyping studies nevertheless support the theory that $C$. hominis and C. parvum behave differently in humans especially with reference to the specificity of the clinical presentation. In C. hominis cases, nongastrointestinal symptoms (e.g., joint pain, eye pain, headache, dizziness and fatigue) are seen more often than in cases of $C$. parvum. Furthermore in young children, infections with C. hominis and, if symptomatic, C. parvum, are often heavy associated with fecal lactoferrin and growth shortfalls. C. hominis appears to stimulate inflammation irrespective of age; this raises important questions regarding how it may specifically induce greater proinflammatory response [29].

\subsection{Transmission Modes and Risk Factors}

1.3.1. Impact of Water Livestock on Transmission. Waterborne contamination is a growing concern causing widespread disease outbreaks. Factors that have contributed to the emergence of cryptosporidiosis in animals include increased environmental contamination and trends in livestock production. In humans the zoonotic nature of infection, along with increased numbers of at-risk population have contributed to the rate intensification of the disease [30]. Risk factors for 
TABLE 1: Factors that affect prevalence and adequate surveillance of cryptosporidiosis.

Epidemiological indexes

Population age

Gender

Individual immunological status

Geographical distribution and ethnic group

Human activities

Hygienic and diet practices

Rural and urban settings

Human waste contamination

Livestock pollution

Water treatment systems; food preparation styles and procedures

Travels, immigration

Environmental and social affecting factors

Animal pollution

Famine, malnutrition, dehydration

Geography, international adoptions

Calamities (typhoons, local wars, floods, etc)

Climate variation, pollution, deforestation and seasonal rains

Under-ascertainment factors in surveillance

Improper sampling of contaminated water systems and food

Difficulty to identify the likely source of infection

Misidentification of outbreak sources

Multiple protozoan coinfections

Under-ascertainment factors in clinics and diagnosis

Poor or diversified symptom presentations and low clinician's sensitivity to consider protozoa as agents of gastrointestinal infections

Limited inclusion of protozoan searching in operational diagnostic workflows

Self-limiting infection course in immunocompetent adults and children

Low inclusion of advanced molecular tools for routine diagnosis

waterborne infections are deduced primarily from outbreak surveillance data. However, in the USA, only a fraction of the estimated water-related outbreaks are reported through passive surveillance [31]. While the outbreak epidemiology due to cryptosporidiosis is still a matter of concern, despite objective definition and identification of outbreak [32], the epidemiology of sporadic (non-outbreak-related) cases is largely unknown. Few papers have reported studies using the Geographical Information System (GIS) methods to map the locations of residences of sporadic cases or to assess ecosystems of cryptosporidiosis [33-36]. In the last few years a plethora of literature has been focusing on the description of advanced molecular markers and technologies [37-41], population structures [42-44], genetic variation of the parasite $[45,46]$, and linkage to its complex epidemiology $[10,47-50]$.

1.3.2. Impact of Climate and Weather on Transmission. A seasonal incidence of infection is sometimes present, possibly corresponding to rainfall peaks, increased pollution from farm waste, or calving and lambing activities [51, 52] (Table 1). Pivotal works have thoroughly investigated the seasonality of cryptosporidiosis also for children, showing highest prevalence from October to March. Such pattern may suggest a possible relationship with child care centre attendance in Europe [53], or provide correlation between seasonality and endemicity in Africa [54]. Recently, a metaanalysis has examined the seasonal patterns of cryptosporidiosis, with relation to precipitation and temperature fluctuations worldwide [55], according to the geographical Köppen Climate Classification [56]. Outcome data were linked to monthly ambient temperature and precipitation for each location and, for the Sub-Saharan Africa, to the Normalized Difference Vegetation Index, a remote sensing measure for the combined effects of temperature and precipitation on vegetation and cryptosporidiosis. Strong seasonal drivers for cryptosporidiosis showed precipitation in moist tropical locations and temperature in mid-latitude and temperate climates [55]. While climatic conditions typically define a pathogen habitat area, meteorological factors affect timing and intensity of seasonal outbreaks. Therefore, seasonality and meteorological forecasts can represent a key indicator and tool, respectively, to plan prevention programs for waterborne cryptosporidiosis (Table 1). 


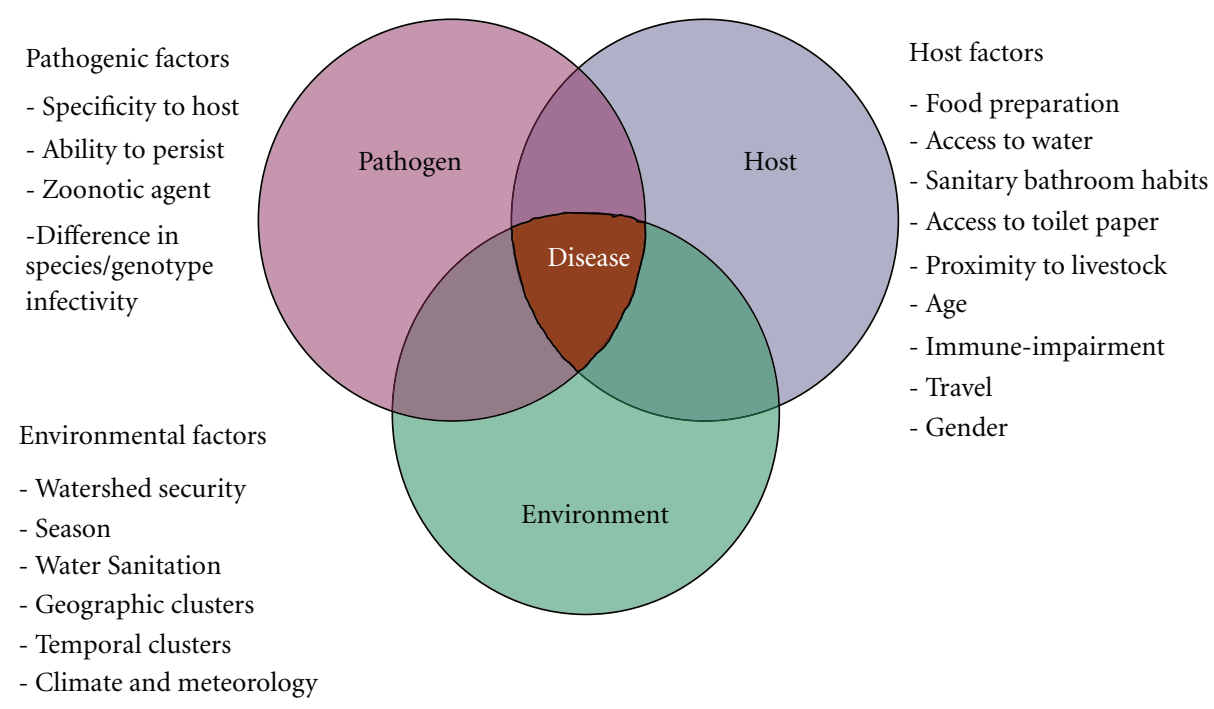

FIgure 3: Venn Diagram of factors leading to Cryptosporidium infection. Parasite, host and environmental indexes acting as key factors for the global burden of cryptosporidiosis. For details see Table 1. Putignani and Menchella, 2010.

1.4. Cryptosporidiosis a "Neglected Disease" and a Poverty Index. In developing countries the impact of protozoan pathogens represents a major cause of gastrointestinal illnesses and is becoming of growing impact, also because new epidemiological markers and indicators of infection are allowing researchers and clinicians to strengthen surveillance programs and diagnostic procedures [36, 44, 57]. However, a large proportion of diarrhoeal illnesses in these countries, especially in children, are still ascribed to an unknown etiology, often because the only available detection methods, such as microscopy and culture used in many areas, have low sensitivity. Particularly, Cryptosporidium and Giardia are still major causes of diarrhoeal diseases of humans worldwide, and are included in the World Health Organisation's Neglected Diseases Initiative [58, 59]. The neglected tropical diseases are often indicators of poverty and disadvantage. Those most affected are the poorest populations often living in remote, rural areas, urban slums, conflict and natural disaster zones, where aggravate conditions are conducive to the spread of these diseases (Table 1). Cryptosporidium accounts for up to $20 \%$ of all cases of childhood diarrhea in developing countries, and is a potentially fatal complication of AIDS [30] and often, in early childhood, is often associated with poor cognitive function and failure to thrive [60].

1.5. Clinical Symptoms As Patognomic Evidence. Variation in symptoms may represent and additional key indicator to set up specific diagnostic workflows for Cryptosporidium detection and to infer correlation between infecting species/subtypes and epidemiology (Table 1) (Figure 3). In the last few years, in low-income countries, an enhanced attention has been directed to the observation of symptom variation, which may provide a successful index for wholesale effective surveillance programs $[50,61]$. A recent extensive study was performed in Bangladesh on 3646 case patients, who presented with diarrhea [61]. The study assessed the proportion of diarrhea cases attributable to $C$. hominis, C. parvum, Entamoeba histolytica, and G. lamblia. Cryptosporidium species and E. histolytica were more prevalent in patients with acute diarrhea, all ages and, specifically, those from 0 to12 months of age. Remarkably, patients with diarrhea and cryptosporidiosis were less likely to have abdominal pain; patients with amebiasis were more likely to have visible blood in stool; patients with giardiasis were more likely to be dehydrated, compared with control subjects [61]. Recently, clinical symptoms such as abdominal pain and/or diarrhoea were selected as key indicators of Cryptosporidium and Giardia infections in patients in Belgium (Table 2) [62].

1.6. Epidemiology of Cryptosporidium Species. C. hominis and C. parvum are the major causing agents of human cryptosporidiosis both in immunocompetent and in immunocompromised individuals but their prevalence varies in different regions of the world [49]. Macroepidemiological analyses showed that $C$. hominis is more prevalent in North and South America, Australia, and Africa, whereas C. parvum causes more human infections in Europe, especially in the UK [49]. Particularly, C. meleagridis can be confirmed as an emerging human pathogen, being responsible for $1 \%$ of all infections in England [49] and about 10\% in Perù, where its prevalence is as high as for C. parvum [50]. A recent important long-term typing overview reported the epidemiology of human cryptosporidiosis in the UK (England and Wales) by analysing 8,000 Cryptosporidium isolates which were submitted for typing from 2000 to 2003 [63] (Table 2). The majority were either C. parvum or C. hominis. Six other known Cryptosporidium species or genotypes were found: C. meleagridis, C. felis, C. canis, and the Cryptosporidium cervine [64], horse and skunk [65] genotypes. This study showed that epidemiology differed among infecting species (Figure 3). C. parvum cases were younger, although $C$. hominis was more prevalent in infants 
TABLE 2: Worldwide distribution of principal sporadic cases and surveillance data reported in the last decade (1998-2008): case characteristics.

\begin{tabular}{|c|c|c|c|c|c|}
\hline $\begin{array}{l}\text { Samples } \\
\text { (surveillance study } \\
\text { or sporadic cases) }\end{array}$ & Country & $\begin{array}{l}\text { Age } \\
\text { (human cases) }\end{array}$ & Technique/genotyping tool & Species/genotypes/subgenotypes ${ }^{1}$ & Reference \\
\hline Human stools & India & Children & $\begin{array}{l}\text { 18S rRNA, SSU, COWP, } \\
\text { Cpgp40/15, TRAP-C1-based } \\
\text { PCR }\end{array}$ & $\begin{array}{l}\text { C. hominis (Ia, Id, Ie, Ib), } \\
\text { C. parvum (Ic), C. felis }\end{array}$ & {$[36]$} \\
\hline $\begin{array}{l}\text { Environmental } \\
\text { (water) }\end{array}$ & China & - & $\begin{array}{l}\text { 18S rRNA PCR-RFLP and } \\
\text { sequence analyses; GP60 }\end{array}$ & $\begin{array}{l}\text { C. hominis (IbA19G2, IbA20G2, } \\
\text { and IbA21G2), Ia, Id, Ie } \\
\text { (IeA12G3T3), If (IfA22G1) } \\
\text { C. meleagridis, C. baileyi, } \\
\text { C. parvum, C. suis, C. muris, rat } \\
\text { genotype, avian genotype } 3\end{array}$ & {$[41]$} \\
\hline Human stools & Perù & Children & GP60 & $\begin{array}{l}\text { C. hominis (Ia, Ib, Id, Ie, Id), } \\
\text { C. parvum (IIc), C. meleagridis, } \\
\text { C. canis, C. felis }\end{array}$ & {$[50]$} \\
\hline Human stools & Ireland & $\begin{array}{l}\text { Adults and } \\
\text { children }\end{array}$ & $\begin{array}{l}\text { 18S rRNA and COWP } \\
\text { PCR-RFLP; GP60 }\end{array}$ & $\begin{array}{l}\text { C. hominis (IbA10G2), } \\
\text { C. parvum (IIaA18G3R1) }\end{array}$ & {$[51]$} \\
\hline Human stools & Belgium & $\begin{array}{l}\text { Adults and } \\
\text { children }\end{array}$ & $\begin{array}{l}\text { 70-kDa heat shock protein, } \\
\text { 60-kDa glycoprotein (GP60) }\end{array}$ & $\begin{array}{l}\text { C. hominis (IbA10G2, IbA9G3) } \\
\text { C. parvum (IIaA15G2R1, } \\
\text { IIcA5G3a, IIdA16G1 } \\
\text { IIaA15G2R1) }\end{array}$ & {$[62]$} \\
\hline Human stools & UK & $\begin{array}{l}\text { Adults and } \\
\text { children }\end{array}$ & $\begin{array}{l}\text { COWPand small sub-unit (SSU) } \\
\text { rRNAgene PCR-RFLP }\end{array}$ & $\begin{array}{l}\text { C. parvum, C. hominis, } \\
\text { C. meleagridis, C. felis, C. canis, } \\
\text { Cryptosporidium cervine, horse, } \\
\text { skunk genotypes }\end{array}$ & {$[63]$} \\
\hline Human stools & Haiti & $\begin{array}{l}\text { Adults and } \\
\text { children }\end{array}$ & 18S rRNAPCR-RFLP & C. hominis, C. parvum, C. felis & [69] \\
\hline Human stools & Perù & Adults & GP60 & $\begin{array}{l}\text { C. hominis (Ia, Ib, Id, Ie) C. canis, } \\
\text { C. felis, C. parvum, C. meleagridis }\end{array}$ & {$[74]$} \\
\hline $\begin{array}{l}\text { Human and } \\
\text { animal stools }\end{array}$ & Portugal & $\begin{array}{l}\text { Adults and } \\
\text { children }\end{array}$ & GP60 & $\begin{array}{l}\text { C. hominis (Ib, If), C. parvum } \\
\text { (IIa, IIb, IIc and IId) }\end{array}$ & {$[78]$} \\
\hline $\begin{array}{l}\text { Environmental } \\
\text { (water) }\end{array}$ & France & - & IMS-IFA ${ }^{2}, 18 \mathrm{~S}$ rRNA PCR-RFLP & C. hominis, C. parvum & {$[96]$} \\
\hline Animal stools & Ireland & Neonatal calves & GP60 & $\begin{array}{l}\text { C. parvum (IIaA18G3R1), } \\
\text { C. bovis, Cryptosporidium } \\
\text { deer-like genotype }\end{array}$ & {$[101]$} \\
\hline $\begin{array}{l}\text { Environmental } \\
\text { (water) }\end{array}$ & Portugal & - & IMS-IFA $^{1}$, PCR & $\begin{array}{l}\text { C. hominis (IdA15) C. parvum } \\
\text { (IIaA15G2R1, IIaA16G2R1, } \\
\text { IIdA17G1) }\end{array}$ & {$[103]$} \\
\hline $\begin{array}{l}\text { Animal and } \\
\text { human stools }\end{array}$ & Portugal & $\begin{array}{l}\text { Adults and } \\
\text { children }\end{array}$ & GP60 & $\begin{array}{l}\text { C. hominis, C. parvum, C. felis, } \\
\text { C. meleagridis }\end{array}$ & {$[104]$} \\
\hline Human stools & MI (USA) & $\begin{array}{l}\text { Adults and } \\
\text { children }\end{array}$ & $\begin{array}{l}18 \mathrm{~S} \text { rRNA and } \\
\text { COWPPCR-RFLP; GP60 }\end{array}$ & $\begin{array}{l}\text { C. hominis, C. parvum (cervine } \\
\text { genotype, cervine genotype } \\
\text { variant, human genotype } \mathrm{W} 17 \text { ) }\end{array}$ & {$[148]$} \\
\hline $\begin{array}{l}\text { Animal and } \\
\text { human stools }\end{array}$ & Iran & $\begin{array}{l}\text { Children and } \\
\text { one adult }\end{array}$ & 18S rRNA PCR-RFLP & $\begin{array}{l}\text { C. parvum, C. hominis } \\
\text { (anthroponotic and zoonotic } \\
\text { genotype) }\end{array}$ & {$[164]$} \\
\hline Animal stools & China & Neonatal calves & $\begin{array}{l}\text { 70-kDa heat shock protein; } \\
18 \mathrm{~S} \text { rRNA, actin-based PCR }\end{array}$ & C. andersoni, C. ryanae & {$[165]$} \\
\hline Animal stools & India & Neonatal calves & 18S rRNA PCR-RFLP & C. parvum, C. hominis & {$[206]$} \\
\hline Human stools & UK & $\begin{array}{l}\text { Adults and } \\
\text { children }\end{array}$ & $\begin{array}{l}\text { SSCP-based analysis of the } \\
18 \text { S rRNA SSU and ITS- } 2 \text { spacer }\end{array}$ & C. parvum (types 1 and 2) & [208] \\
\hline Human stools & UK & Adults & GP60 & C. hominis (IbA10G2) & [209] \\
\hline Human stools & $\begin{array}{l}\text { Kenya, } \\
\text { Malawi, } \\
\text { Brazil, } \\
\text { Vietnam, UK }\end{array}$ & $\begin{array}{l}\text { Adults and } \\
\text { children }\end{array}$ & 18S rRNA PCR-RFLP & $\begin{array}{l}\text { C. parvum (human genotype), } \\
\text { C. parvum (bovine genotype), } \\
\text { C. meleagridis, C. muris }\end{array}$ & [222] \\
\hline
\end{tabular}


TABle 2: Continued.

\begin{tabular}{|c|c|c|c|c|c|}
\hline $\begin{array}{l}\text { Samples } \\
\text { (surveillance study } \\
\text { or sporadic cases) }\end{array}$ & Country & $\begin{array}{l}\text { Age } \\
\text { (human cases) }\end{array}$ & Technique/genotyping tool & Species/genotypes/subgenotypes ${ }^{1}$ & Reference \\
\hline Human stools & $\begin{array}{l}\text { Switzerland, } \\
\text { Kenya, USA }\end{array}$ & $\begin{array}{l}\text { Adults and } \\
\text { children }\end{array}$ & $\begin{array}{l}\text { 18S rRNA; HSP-70; acetyl } \\
\text { coenzyme A synthetase }\end{array}$ & $\begin{array}{l}\text { C. parvum ("human" genotype, } \\
\text { "cattle" genotype), C. felis, } \\
\text { C. meleagridis }\end{array}$ & {$[223]$} \\
\hline Human stools & Spain & $\begin{array}{l}\text { Adults and } \\
\text { children }\end{array}$ & $\begin{array}{l}\text { 18S rRNA-, COWP-based } \\
\text { PCR-RFLP }\end{array}$ & $\begin{array}{l}\text { C. hominis, C. parvum, } \\
\text { C. meleagridis, C. felis }\end{array}$ & {$[224]$} \\
\hline Human stools & $\begin{array}{l}\text { Equatorial } \\
\text { Guinea }\end{array}$ & $\begin{array}{l}\text { Adults and } \\
\text { children }\end{array}$ & COWP-based PCR-RFLP & $\begin{array}{l}\text { C. parvum, C. hominis, } \\
\text { C. meleagridis }\end{array}$ & {$[232]$} \\
\hline $\begin{array}{l}\text { Human and } \\
\text { animal stools }\end{array}$ & Thailand & Adults & 18S rRNA PCR & C. parvum & [239] \\
\hline Human stools & Perù & $\begin{array}{l}\text { Adults and } \\
\text { children }\end{array}$ & 18S rRNA-based PCR-RFLP & $\begin{array}{l}\text { C. hominis, C. meleagridis, } \\
\text { C. parvum, C. canis, C. felis, } \\
\text { Cryptosporidium (pig genotype) }\end{array}$ & {$[240]$} \\
\hline Human stools & Poland & $\begin{array}{l}\text { Adults and } \\
\text { children }\end{array}$ & COWP and $\beta$-tubulin-based PCR & $\begin{array}{l}\text { C. hominis, C. parvum, } \\
\text { C. meleagridis, }\end{array}$ & {$[254]$} \\
\hline Human stools & Madagascar & Children & GP60 & $\begin{array}{l}\text { C. hominis (Ia, Id, Ie), C. parvum } \\
\text { (IIc) }\end{array}$ & {$[275]$} \\
\hline
\end{tabular}

${ }^{1}$ When available, reported subgenotypes are the most common detected.

${ }^{2}$ IMS-IFA, immunomagnetic separation followed by immunofluorescence assay: Method 1623 of the USA Environmental Protection Agency (USEPA).

under one year and in females aged 15 to 44 years. Spring peaks were due to C. parvum, while C. hominis was more prevalent during the late Summer and early Autumn as well as in patients reporting recent travel abroad [63] (Table 1) (Figure 3) (Table 2). C. parvum and C. hominis are two species responsible for most human cases of cryptosporidiosis. The relationship between the global population structure of these species and the host population arrangement was thoroughly investigated by the study of Tanriverdi et al., 2008 [66], in which a series of worldwide C. parvum and C. hominis isolates were genotyped. Geographical partitions or patterns for both parasite species were observed among the countries (Uganda, Serbia, Turkey, Israel, UK, USA, and New Zealand), possibly because of different prevailing ecological determinants of transmission [66]. Rather than conforming to a strict paradigm of either a clonal or a panmictic population structure [43], these data seem to suggest a flexible reproductive strategy characterized by the cooccurrence of both propagation patterns.

A predominance of $C$. hominis was observed in persons in developing countries, such as pediatric populations from Perù [26, 50], Malawi [67], Kenya [68], India [36], Haiti [69], and Brazil [70], children and elderly persons from South Africa [71], and hospitalized HIV-infected children from South Africa [72] and Uganda [73]. A comparatively large proportion of participants infected with $C$. meleagridis was observed in a wide community in Haiti [69], a finding that was also reported at a high frequency in HIV-infected adults in Perù [74]. This species has been reported, even if rarely, by other studies regarding either children or adults with or without HIV infection from other geographical places as Portugal [75], India [36, 57], Taiwan [76], or Iran [77].

Environmental isolates of Cryptosporidium from China were thoroughly investigated in the study of Feng et al., 2009 (Table 2) [41]. Interestingly, the predominant species was C. hominis followed by C. meleagridis. The other Cryptosporidium species/genotypes identified included C. baileyi, C. parvum, C. suis, C. muris, rat genotype, avian genotype 3 , and a novel genotype. The Ib identified subtypes (Table 2) [41], were very different from the subtypes IbA9G3 and IbA10G2 commonly found in other areas of the world. The IbA9G3 is usually observed in humans in Kenya, India, and Australia, and IbA10G2 is commonly seen in South Africa, Perù, USA, Canada, Australia, and European countries, as France, UK, Portugal, Spain and Ireland [50, 68, 72, 78-84]; IbA10G2 is responsible for more than half of the waterborne outbreaks in USA, and Canada $[85,86]$. Likewise, the IeA12G3T3 was different from the most common IeA11G3T3 subtype, although observed also in Louisiana, Australia, and Jamaica $[79,87,88]$ (Table 2). Also the IfA20G1 and IfA22G1 subtypes were detected, as previously in children in South Africa [72], occasionally in HIV-positive adults in Portugal [78] and in India [89], but not in most other studies, supporting the presence of unique transmission of C. hominis in China (Table 2).

In spite of the availability of substantial sequence data obtained by reliable typing tools (e.g., COWP, TRAP C-1, GP60) there is still no comprehensive analysis of the genetic richness, and diversity within $C$. hominis and C. parvum [90]. The worldwide literature produced so far highlights the need to pursue on detailed molecular epidemiological studies (e.g., GP60), especially in "neglected" geographical regions and from a wide range of hosts species. Indeed researchers have to address the key question if the low diversity associated to the substantial richness of the GP60 locus is due to the genetics of the organism or to a lack of data from countries with potential endemic transmissions (Africa, South east, China, and India subcontinents) [90]. High-throughput technologies ("genome sequence surveys") and advanced bioinformatic platforms 
(http://cryptodb.org/cryptodb/) may allow unprecedented comparative studies of Cryptosporidium isolates and overcome the limits of incomplete global epidemiological data.

1.7. Diagnostic Pitfalls: The Case of a Developed Country. An interesting case-control study [91], performed in Lower Saxony (Germany), during 2001 to 2005, reported 744 cases of cryptosporidiosis detected by the Governmental Institute of Public Health. The study demonstrated that a broad and improved diagnostic activity in reference laboratories was able to better describe cryptosporidiosis, reflecting the real occurrence of this infection, often underestimated. The yearly incidence rate of 1.9 notified cases per 100000 population within Lower Saxony exceeded the German mean incidence rate of 1.5. In several neighbouring districts there was a striking heterogeneity of regional incidences. However, highest rates of notification were associated with one particular laboratory where all stool samples, submitted for routine microbiological diagnosis, were screened for C. parvum. Diagnostic work was done by valid, specific, CE-certified procedures. The inferred conclusion was that the increased regional incidence rate was caused by the extensive diagnostic activity of this laboratory, presuming an underestimation in other regions [91]. These data seem to suggest that, even in high-income countries, routine diagnostic protocols should be thoroughly integrated by highly advanced identification workflows.

1.8. Aims of the Review. This review aims to discuss the updated global distribution of cryptosporidiosis, focusing on the main records reported for sporadic cases and outbreaks in the last decade and exploiting spatial and temporal determinants of infection particularly for low-income countries. With this intent, key indicators were critically considered to describe dynamics of transmission linked to the principal reservoirs of environmental infection (e.g., water, food) but also to the main host factors (e.g., age, travel, immunostatus) (Table 1) (Figure 3).

The principal cryptosporidiosis ecosystems, linked to outbreak and sporadic human cases, were geographically mapped worldwide (Figure 4) and origins of infection were categorised as Waterborne (Section 3), Foodborne (Section 4), Travelers' (Section 5), and HIV-related disease (Section 6). Cryptosporidiosis in children was separately approached, according to multivariate and specific exposure factors acting in this life age (Section 7). The set of reviewed data (outbreak and sporadic human cases, environmental and veterinary surveillance reports) was correlated, in each Section, to the geographical setting (continent) and subsetting (country) (Figure 4, Figure 5).

\section{Study Approach}

International surveillance networks and suites of free and open-source for epidemiology control or open-access peerreviewed journals about infectious diseases surveillance prevention and control in Europe, Canada, USA, and Australia were exploited for our analysis (Table 3).
The MEDLINE database (http://www.ncbi.nlm.nih.gov/ sites/entrez) was searched for the following terms: Cryptosporidium, cryptosporidiosis, water- and foodborne outbreak, travelers' cryptosporidiosis, HIV cryptosporidiosis, children cryptosporidiosis. More than 400 papers were found to be eligible after reading the full text and/or abstract. Of these potentially adequate articles, 300 fulfilled the inclusion criteria: (i) presence of rate data of cryptosporidiosis within the last decade; (ii) presence of geographical distribution and transmission data for relevant sporadic cases and outbreak occurred in the last decade; (iii) observation of potential environmental factors affecting and/or worsening the occurrence of parasite transmission (Table 1) (Figure 3).

\section{Cryptosporidiosis a "Waterborne Disease"}

3.1. Outbreaks Associated to Protozoan Parasites. C. parvum accounts for the majority of 325 water-associated outbreaks of parasitic protozoan diseases recently worldwide reported (165 out of 325, 50.8\%), immediately followed by Giardia duodenalis (132 out of $325,40.6 \%)$ and afterwards by $E$. histolytica ( 9 out of $325,2.8 \%$ ), Cyclospora cayetanensis (6 out of 325, 1.8\%), and in the least by Toxoplasma gondii and Isospora belli ( 3 out of 325, 0.9\% each), Blastocystis hominis ( 2 out of $325,0.6 \%$ ) and Balantidium coli, the microsporidia, Acanthamoeba and Naegleria fowleri (1 out of 325, 0.3\% each) [6].

Out of the 71 Cryptosporidium-linked outbreaks described in the last decade, 40 (56.3\%) appear to be correlated to waterborne diseases, with a distribution almost constant throughout the years, marked by picks in 2000, 2001, 2002, and 2007. Geographically, the outbreaks seem to be concentrated in the USA, Canada, Australia and in Europe, especially in the UK and Ireland and appear to affect both adults and children (Table 4) (Figure 4). Worldwide environmental and veterinary surveillance data reveal the presence of Cryptosporidium spp. in the entire watertreatment system, which represents an unacceptable health risk, particularly in sensitive (pregnant women, children) and immunocompromised populations (HIV-positive and transplanted patients). Such evidence suggests that focus ought to be placed on prevention of human and animal waste contamination especially in authorized recreational waters, and in a few cases also in well-maintained community swimming pools treated by supplemental disinfection treatment. Remarkably, cryptosporidiosis is nowadays the most frequently reported gastrointestinal illness in outbreaks associated with treated (disinfected) recreational water venues in USA [92]. However, microbiological surveillance should be also directed to other fresh waters, affected by occasional peaks of contamination, owing to heavly rainfall going through fields to lakes, or by high-contamination events that might occur upstream from the sampling river sites irrigation. In USA, where large episodes of cryptosporidiosis are detected, public water treatments should steadily adhere to USA Environmental Protection Agency (USEPA) regulations to improve water quality, aquatic venue design, usage, and maintenance and to update the Center for diseases control and prevention (CDC) 

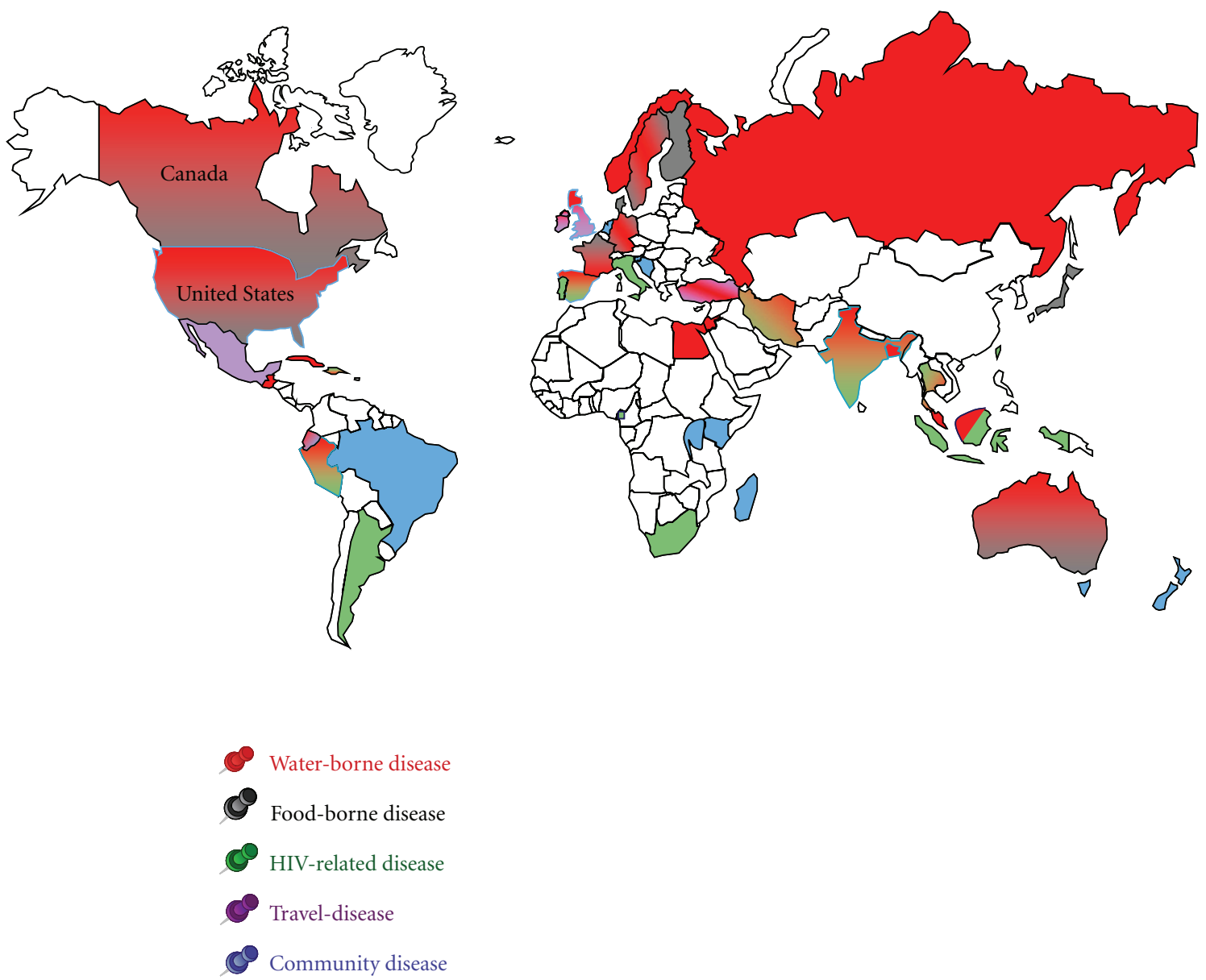

FIGURE 4: Geography of worldwide occurrence of human cryptosporidiosis outbreaks and sporadic cases. A color-coded distribution of the main cases of cryptosporidosis reported in the literature during the last decade (1998-2008) for the entire population (adults and children) is here represented. Waterborne and foodborne diseases are represented by red and grey color, respectively. Spreading of the infection due to HIV immunological impairment is represented by green and travel-related disease by pink color. When not applicable the definition of waterborne and foodborne disease, the term community disease has been applied to person-to-person contacts and represented by a pale blue color. For countries characterised by two or three coexisting transmission modes, a double color-filling effect plus thick border lines have been used, consistently with the above reported code. Putignani and Menchella, 2010.

surveillance programs with periodical reports on etiologic agents, failures of water-treatment systems, and deficiencies associated with outbreak management [93] (Table 3). In developed countries, the detection of Cryptosporidium and Giardia should be an integral part of the quality system in the water industry and multidisciplinary approaches among public health professionals (epidemiologists, clinicians and parasitologists) should be routinely included to establish priorities in public health prevention programs and to design appropriate operational workflows for both detection and diagnosis. In developing countries the potential of infection is enhanced by the absence of sanitary and parasitological drinking water monitoring. Moreover the burden of the infection is surely underestimated for the small number of appropriate surveillance programs and for the absence of suitable diagnostic algorithms.

3.2. Waterborne Cryptosporidiosis in Europe. Recently, in Europe, the circulation of Cryptosporidium spp. populations has been thoroughly investigated because of the improved surveillance and diagnosis of both sporadic cases and outbreaks of cryptosporidiosis (Table 4). From a waterborne outbreak of diarrhea in France, the $91 \%$ of the isolates of the parasite were characterised as C. hominis type Ib [86], consistently with the current idea that $\mathrm{Ib}$ is the predominant allele associated with waterborne cryptosporidiosis worldwide $[79,81]$.

Earlier evidence had suggested that accidental ingestion of natural waters while bathing carries a risk of infection by waterborne protozoa both in UK and USA $[94,95]$. In order to evaluate this risk in France, a one-year prospective study on recreational lakes and river sites located near Paris, chosen for frequent bathing and boating, was undertaken (Table 2) [96]. Giardia cysts and Cryptosporidium oocysts were detected in the recreational lakes with occasional peaks and in the river sites throughout all the year. Genetic characterization of Cryptosporidium revealed the presence of both C. hominis and C. parvum species (Table 2). Based on 


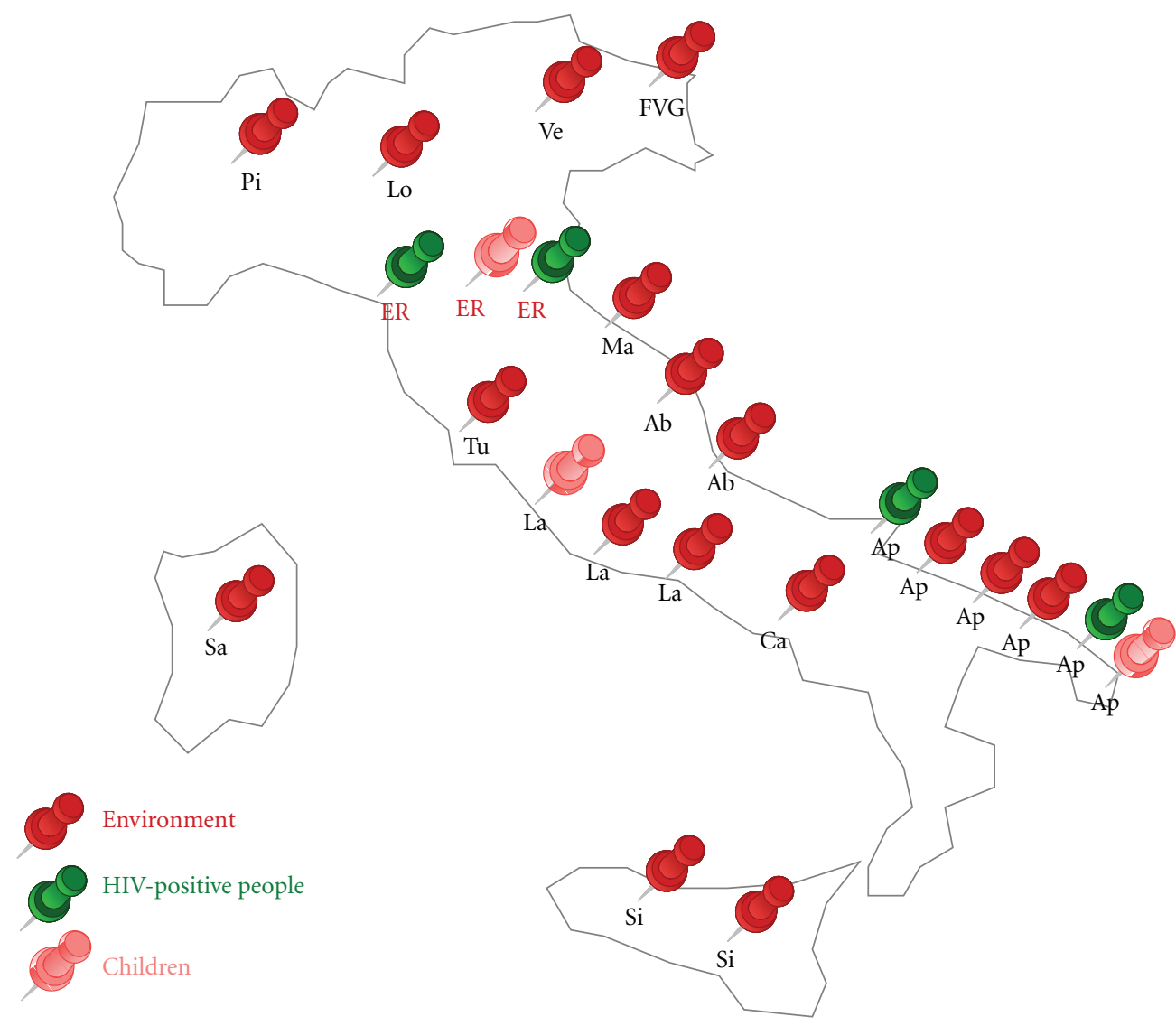

Figure 5: Geographical distribution of Italian studies on Cryptosporidium surveillance. A map of the principal surveillance studies performed on environmental and human samples is here reported by using the following color codes: red for environmental samples (water, animal); green for human samples associated to HIV in adults; pink for children samples. Symbols refer to different Italian regions: Ab, Abruzzo; Ap, Apulia; Ca, Campania; ER, Emilia Romagna; FVG, Friuli Venezia Giulia; La, Latium; Lo, Lombardia; Ma, Marche; Pi, Piedmont; Sa, Sardinia; Si, Sicily; Tu, Tuscany; Ve, Veneto. Putignani and Menchella, 2010.

a model for quantitative microbial risk assessment (QMRA), the study confirmed that bathing in surface waters was actually associated with a significant risk of infection by Cryptosporidium or Giardia. This was especially the case for rivers not protected from human or animal fecal contamination. Surface waters, especially in rural areas, may be soiled by contaminated farmyard manure or slurry used as fertiliser for crop cultivation. Pasturing of infected livestock near crops or defaecation of infected undomesticated hosts onto them is an important factor for zoonotic contamination [7]. Also sludges, night soil, and raw waters may contribute to worldwide water pollution [97]. Recently, as inferred by outbreak surveillance data, recreational, drinking and fountain waters have been identified as important source of community infections worldwide (Table 4) [6]. In Ireland, as several recent waterborne outbreaks have shown (Table 4) [80, 98, 99] and as thoroughly discussed by the latest paper of Cheng et al., 2009 on the presence of Cryptosporidium and Giardia in wastewater treatment plants [100], cryptosporidiosis poses a significant threat to public health. In a recent study [51], performed on human stool samples collected in diversified geographical areas of Ireland, Cryptosporidium spp. were genetically characterised
(Table 2). Overall, C. parvum was identified in $80 \%$ and C. hominis in $20 \%$ of cases, with an higher proportion in older age groups. C. parvum was the most common species in the rural, more sparsely populated West of Ireland and exhibited a pronounced Spring peak coincident with the peak of the national cryptosporidiosis incidence rate. The most common C. parvum subgenotype (Table 2) was the same detected in Irish cattle [101] confirming the prevalence of zoonotic Cryptosporidium transmission in Ireland.

In Holland, water in canals and recreational lakes are contaminated through the discharge of raw sewage from houseboats, sewage effluent, dog and bird feces. During two successive one-year study periods, the water quality in canals and recreational lakes was tested in Amsterdam with regard to the presence of fecal indicators and waterborne pathogens [102]. Cryptosporidium oocysts and Giardia cysts were detected both in canals and recreational lakes, despite conformity with the European bathing water legislation, indicating these parasites as health risk pathogens for situations of exposure to surface waters [102].

A significant study was undertaken to monitor the presence of Cryptosporidium and Giardia in water samples, including raw and treated waters from both surface and 
Table 3: Public data sources exploited in the current study.

\begin{tabular}{|c|c|c|}
\hline Site name & Link & Reference \\
\hline \multicolumn{3}{|c|}{ International surveillance networks } \\
\hline $\begin{array}{l}\text { Neglected Diseases Initiative of the World } \\
\text { Health Organization }\end{array}$ & http://www.who.int/neglected_diseases/en/ & {$[59]$} \\
\hline $\begin{array}{l}\text { Center for Disease Control and } \\
\text { Prevention }\end{array}$ & http://emergency.cdc.gov/agent/agentlist-category.asp & {$[93]$} \\
\hline $\begin{array}{l}\text { San Francisco Bay Area Cryptosporidiosis } \\
\text { Surveillance Project }\end{array}$ & http://www.sfphes.org/water/index_crypto.htm & {$[136]$} \\
\hline $\begin{array}{l}\text { C-EnterNet (Canadian Integrated Enteric } \\
\text { Disease Surveillance System) }\end{array}$ & http://www.phac-aspc.gc.ca/c-enternet/index-eng.php & [153] \\
\hline Public Health Agency of Canada & http://www.phac-aspc.gc.ca/index-eng.php & {$[154]$} \\
\hline FoodNet & http://www.cdc.gov/FoodNet/ & {$[183]$} \\
\hline \multicolumn{3}{|c|}{ Suites of free and open-epidemiology data source } \\
\hline Tri-County Health Department & http://www.tchd.org/ & {$[150]$} \\
\hline AIDS site & http://data.unaids.org/en/default/asp & {$[161]$} \\
\hline Communicable Diseases Branch & http://www.health.qld.gov.au/ph/cdb/default.asp & {$[245]$} \\
\hline NetEpi & http://code.google.com/p/netepi & {$[273]$} \\
\hline Epi Info software & http://www.who.int/chp/steps/resources/EpiInfo/en/index.html & {$[274]$} \\
\hline \multicolumn{3}{|c|}{ Open-access peer-reviewed journals } \\
\hline $\begin{array}{l}\text { Euroserveillance Europe's Journal on } \\
\text { infectious diasese epidemiology, } \\
\text { prevention and control }\end{array}$ & http://www.eurosurveillance.org/ & $\begin{array}{l}{[63,81,98} \\
99,178,246- \\
248,276,291 \\
292]\end{array}$ \\
\hline $\begin{array}{l}\text { Center for Disease Control and } \\
\text { Prevention: Morbidity and Mortality } \\
\text { Weekly Report (MMWR) }\end{array}$ & http://www.cdc.gov/mmwr/ & $\begin{array}{c}{[92,131,132,} \\
146,156, \\
185-191,249 \\
258,260,285 \\
293-295]\end{array}$ \\
\hline Public Health Agency of Canada & http://www.phac-aspc.gc.ca/surveillance-eng.php & {$[86,296,297]$} \\
\hline
\end{tabular}

ground sources in Portugal [103]. C. parvum was the most common detected species, followed by $C$. hominis, $C$. andersoni, and C. muris. These results are clearly suggestive of a wide distribution of Cryptosporidium spp. in source and treated waters in Portugal, with high occurrence of humanpathogenic Cryptosporidium genotypes (Table 2) [103, 104].

An important survey of sewage influent samples from 40 Sewage Treatment Works (STWs) throughout Norway were examined for Cryptosporidium oocysts and G. duodenalis cysts [105]. The data propose giardiasis as more widespread, and occurring with greater infection intensity than cryptosporidiosis: for Cryptosporidium, the highest estimate was up to 5 per 100000 individuals in Eastern Norway while for Giardia 40 per 100000 persons in Western Norway. Removal efficiencies at two STW with secondary treatment processes were estimated to be approximately $50 \%$ for Cryptosporidium and $>80 \%$ for Giardia. A STW with minimal treatment had negligible removal of both parasites. Because many STW in Norway have indeed minimal treatment and discharge effluent into rivers and lakes [105], thus, risk of contamination of water courses by Cryptosporidium and Giardia represents a considerable risk in this country. Contamination from sewage discharges and wild or domestic animals are also important sources for untreated waters [97]. Both Cryptosporidium and Giardia are frequently found in the stool of domestic ruminants, especially young animals. Wild ruminants may serve as reservoirs for these zoonotic parasites, as inferred from an important cross-sectional survey conducted in Belgium to estimate the occurrence of Cryptosporidium and Giardia in captive wild young ruminants. The Cryptosporidium prevalence was $7.5 \%$ in the zoo animals and $3.7 \%$ in the bison from a commercial breeding farm [106].

In the Russian Federation, Cryptosporidium has been recently included in the Index of the Epidemic Safety of Drinking Water as new emerging pathogen, suggesting a growing attention to the parasite control also in geographical areas with no previous surveillance programs and dedicated studies [107].

3.2.1. Waterborne Cryptosporidodis in Italy. In Italy, an intense debate on the epidemiological and public health aspects of Cryptosporidium and Giardia infections has involved many researchers and has lead to investigate infection prevalence data especially on environmental and animal samples but less on human samples because cryptosporidiosis is not a notifiable disease in Italy [108-110] (Figure 5). Protocols recommended by the National Institute of Health are mainly used for detection of these protozoa 
TABLE 4: Worldwide distribution of principal waterborne, foodborne, travel-related, and community outbreaks reported in the last decade (1998-2008): case characteristics.

\begin{tabular}{|c|c|c|c|c|c|c|}
\hline $\begin{array}{l}\text { Outbreak }^{1} \\
\text { type }\end{array}$ & Country & $\mathrm{Ill}^{2}$ & Age & $\begin{array}{c}\text { Likely causes for } \\
\text { outbreak occurring }\end{array}$ & $\begin{array}{l}\text { Species } \\
\text { /genotype }\end{array}$ & Reference \\
\hline \multicolumn{7}{|c|}{2008} \\
\hline Foodborne & Finland & $\begin{array}{l}72 \text { personnel of the } \\
\text { Public Works } \\
\text { Department in } \\
\text { Helsinki }\end{array}$ & Adults & Salad mixture suspected & C. parvum & {$[178]$} \\
\hline Foodborne & Sweden & $\begin{array}{l}21 \text { guests and staff } \\
\text { at a wedding } \\
\text { reception }\end{array}$ & Adults & $\begin{array}{c}\text { Sauce containing } \\
\text { chopped fresh parsley }\end{array}$ & C. parvum & {$[179]$} \\
\hline \multicolumn{7}{|c|}{2007} \\
\hline Waterborne & Norway & 89 hotel guests & Adults & $\begin{array}{l}\text { In-house water } \\
\text { contamination }\end{array}$ & C. parvum & {$[298]$} \\
\hline Community & $\begin{array}{l}\text { Scotland } \\
\text { (UK) }\end{array}$ & $\begin{array}{l}6 \text { veterinary } \\
\text { students }\end{array}$ & Young adults & $\begin{array}{l}\text { Lapse in hygiene, } \\
\text { especially handwashing }\end{array}$ & $\begin{array}{c}\text { C. parvum } \\
\text { (subgenotype } \\
\text { IIaA19G2R1) }\end{array}$ & {$[299]$} \\
\hline Waterborne & $\begin{array}{l}\text { England } \\
\text { (UK) }\end{array}$ & $\begin{array}{l}57 \text { swimming pool } \\
\text { visitors }\end{array}$ & $\begin{array}{l}\text { Children and } \\
\text { adults }\end{array}$ & $\begin{array}{l}\text { Swimming pool } \\
\text { contamination }\end{array}$ & $\begin{array}{l}\text { C. parvum } \\
\text { C. hominis }\end{array}$ & {$[246]$} \\
\hline $\begin{array}{l}\text { Waterbornel } \\
\text { foodborne }\end{array}$ & Germany & 201 soldiers & Young adults & $\begin{array}{l}\text { Tap water/food } \\
\text { contamination in a } \\
\text { military field exercise }\end{array}$ & $\begin{array}{l}\text { C. parvum } \\
\text { (genotype 2) }\end{array}$ & {$[300]$} \\
\hline Waterborne & Ireland (UK) & 182 & Adults & $\begin{array}{l}\text { Contamination of } \\
\text { treated water }\end{array}$ & $\begin{array}{l}\text { C. hominis } \\
\text { C. parvum }\end{array}$ & {$[98]$} \\
\hline Waterborne & Sweden & $800-1000$ & $\begin{array}{l}\text { Children and } \\
\text { adults }\end{array}$ & $\begin{array}{c}\text { Contamination of an } \\
\text { outdoor swimming-pool }\end{array}$ & C. parvum & {$[250]$} \\
\hline Waterborne & ID (USA) & 50 park visitors & $\begin{array}{l}\text { Children and } \\
\text { adults }\end{array}$ & $\begin{array}{c}\text { Exposure to water from } \\
\text { a splash feature }\end{array}$ & $\begin{array}{c}\text { C. hominis } \\
\text { (subgenotype } \\
\text { IaA28R4) }\end{array}$ & {$[258]$} \\
\hline \multicolumn{7}{|c|}{2006} \\
\hline Waterborne & $\begin{array}{l}\text { England } \\
\text { (UK) }\end{array}$ & 35 school people & $\begin{array}{l}\text { Children and } \\
\text { adults }\end{array}$ & $\begin{array}{c}\text { Surface water } \\
\text { contamination during a } \\
\text { farm visit }\end{array}$ & C. parvum & {$[251]$} \\
\hline Waterborne & CO (USA) & $\begin{array}{l}21 \text { attendes to a } \\
\text { pool party }\end{array}$ & $\begin{array}{l}\text { Children and } \\
\text { adults }\end{array}$ & $\begin{array}{l}\text { Swimming, pool } \\
\text { contamination }\end{array}$ & $\begin{array}{l}\text { C. hominis } \\
\text { (subgenotype } \\
\text { IbA10G2) }\end{array}$ & {$[85]$} \\
\hline $\begin{array}{l}\text { Travelers' } \\
\text { infection }\end{array}$ & FL (USA) & 29 retired people & Elderly & $\begin{array}{l}\text { Environmental } \\
\text { contamination with } \\
\text { animal feces }\end{array}$ & $\begin{array}{c}\text { C. parvum } \\
\text { (subgenotype } \\
\text { IIaA16G1R1b) }\end{array}$ & [207] \\
\hline Foodborne & ME (USA) & 14 people & Not reported & Unknown & $\begin{array}{l}\text { Cryptosporidium } \\
\text { spp. }\end{array}$ & $\begin{array}{l}\text { http://www.cdc.gov/ } \\
\text { foodborneoutbreaks/ }\end{array}$ \\
\hline Foodborne & PA (USA) & 2 people & Not reported & Unknown & $\begin{array}{l}\text { Cryptosporidium } \\
\text { spp. }\end{array}$ & outbreak_data.htm \\
\hline Waterborne & FL (USA) & 9 children $^{3}$ & 4 years $^{4}$ & $\begin{array}{l}\text { Water fountain } \\
\text { contamination }\end{array}$ & $\begin{array}{l}\text { Cryptosporidium } \\
\text { spp. }\end{array}$ & {$[261]$} \\
\hline Foodborne & Japan & $\begin{array}{l}4 \text { company } \\
\text { members }\end{array}$ & Adults & $\begin{array}{c}\text { Contamination of raw } \\
\text { meat dish }\end{array}$ & $\begin{array}{c}\text { C. parvum } \\
\text { (genotype IIa) }\end{array}$ & {$[301]$} \\
\hline \multicolumn{7}{|c|}{2005} \\
\hline Foodborne & Denmark & $\begin{array}{l}99 \text { company } \\
\text { employees }\end{array}$ & Adults & Buffet salad eating & C. hominis & {$[180]$} \\
\hline Community & $\begin{array}{l}\text { Scotland } \\
\text { (UK) }\end{array}$ & 62 people & $\begin{array}{l}\text { Adults and } \\
\text { children }\end{array}$ & $\begin{array}{l}\text { Outbreak linked to a } \\
\text { wildlife centre visit }\end{array}$ & C. parvum & {$[247]$} \\
\hline Waterborne & Wales (UK) & 100 & $\begin{array}{l}\text { Mostly young } \\
\text { adults }\end{array}$ & $\begin{array}{l}\text { Contamination of raw } \\
\text { and treated water }\end{array}$ & C. hominis & {$[291]$} \\
\hline Waterborne & Turkey & 191 inhabitants $^{5}$ & $\begin{array}{l}\text { Children and } \\
\text { adults }\end{array}$ & $\begin{array}{l}\text { Contamination of water } \\
\operatorname{tank}\end{array}$ & C. parvum & {$[276]$} \\
\hline
\end{tabular}


Table 4: Continued.

\begin{tabular}{|c|c|c|c|c|c|c|}
\hline $\begin{array}{l}\text { Outbreak }^{1} \\
\text { type }\end{array}$ & Country & $\mathrm{Ill}^{2}$ & Age & $\begin{array}{c}\text { Likely causes for } \\
\text { outbreak occurring }\end{array}$ & $\begin{array}{c}\text { Species } \\
\text { /genotype }\end{array}$ & Reference \\
\hline \multicolumn{7}{|c|}{$2005-2004$} \\
\hline Community & Spain & $\begin{array}{c}24 \text { day-care } \\
\text { children }\end{array}$ & Children & Children diaper use & C. hominis & {$[252]$} \\
\hline \multicolumn{7}{|c|}{2004} \\
\hline Waterborne & Norway & $115^{6}$ & Adults & $\begin{array}{l}\text { Water supply } \\
\text { contamination }\end{array}$ & $\begin{array}{l}\text { C. parvum } \\
\text { (bovin } \\
\text { genotype, } \\
\text { genotype 2, } \\
\text { cervine } \\
\text { genotype) }\end{array}$ & {$[277]$} \\
\hline Community & Croatia & $\begin{array}{l}\text { One family } \\
\text { members }\end{array}$ & $\begin{array}{l}\text { Elderly and } \\
\text { adults }\end{array}$ & $\begin{array}{l}\text { Nosocomial and } \\
\text { person-to-person } \\
\text { contamination }\end{array}$ & C. hominis & {$[302]$} \\
\hline Waterborne & CA (USA) & $\begin{array}{l}273 \text { park } \\
\text { attendants }\end{array}$ & $\begin{array}{l}\text { Children and } \\
\text { adults }\end{array}$ & $\begin{array}{l}\text { Contamination of a } \\
\text { water park }\end{array}$ & $\begin{array}{l}\text { C. parvum } \\
\text { (genotype IIc) }\end{array}$ & {$[127]$} \\
\hline Foodborne & NY (USA) & 212 people $^{7}$ & Not reported & $\begin{array}{c}\text { Contamination of } \\
\text { unpasteurized apple } \\
\text { cider }\end{array}$ & $\begin{array}{l}\text { Cryptosporidium } \\
\text { spp. }\end{array}$ & $\begin{array}{l}\text { http://www.cdc.gov/ } \\
\text { foodborneoutbreaks/ } \\
\text { outbreak_data.htm }\end{array}$ \\
\hline \multicolumn{7}{|c|}{2003} \\
\hline Waterborne & $\begin{array}{l}\text { Yorkshire and } \\
\text { The Humber } \\
\text { (UK) }\end{array}$ & $\begin{array}{c}66 \text { people } \\
\text { attending at the } \\
\text { pool }\end{array}$ & $\begin{array}{l}\text { Children and } \\
\text { adults }\end{array}$ & $\begin{array}{l}\text { Contamination of water } \\
\text { at a public pool }\end{array}$ & $\begin{array}{l}\text { Cryptosporidium } \\
\text { spp. }\end{array}$ & [293] \\
\hline Waterborne & $\begin{array}{l}\text { South West } \\
\text { (UK) }\end{array}$ & $\begin{array}{l}21 \text { children } \\
\text { attending at the } \\
\text { water park }\end{array}$ & Children & $\begin{array}{l}\text { Leisure facility of a } \\
\text { 'water splash zone }\end{array}$ & $\begin{array}{l}\text { Cryptosporidium } \\
\text { spp. }\end{array}$ & [249] \\
\hline Waterborne & $\begin{array}{l}\text { South East of } \\
\text { England } \\
\text { (UK) }\end{array}$ & $\begin{array}{l}17 \text { people } \\
\text { attending at the } \\
\text { pool }\end{array}$ & $\begin{array}{l}\text { Children and } \\
\text { adults }\end{array}$ & $\begin{array}{l}\text { Contamination of water } \\
\text { at a public pool }\end{array}$ & $\begin{array}{l}\text { Cryptosporidium } \\
\text { spp. }\end{array}$ & [249] \\
\hline Waterborne & $\begin{array}{l}\text { Midlands } \\
\quad(\mathrm{UK})\end{array}$ & $\begin{array}{l}122 \text { people } \\
\text { attending at the } \\
\text { park }\end{array}$ & $\begin{array}{l}\text { Children and } \\
\text { adults }\end{array}$ & $\begin{array}{c}\text { Contamination of a } \\
\text { fountain water in a } \\
\text { public park }\end{array}$ & $\begin{array}{l}\text { Cryptosporidium } \\
\text { spp. }\end{array}$ & [249] \\
\hline Waterborne & $\begin{array}{l}\text { South West of } \\
\text { England } \\
\text { (UK) }\end{array}$ & $\begin{array}{l}63 \text { people } \\
\text { attending at the } \\
\text { animal centre }\end{array}$ & Children & $\begin{array}{c}\text { Interactive water feature } \\
\text { at an animal attraction } \\
\text { centre }\end{array}$ & $\begin{array}{c}\text { C. parvum } \\
\text { (genotype 2) }\end{array}$ & {$[248]$} \\
\hline Community & Wales (UK) & $\begin{array}{l}17 \text { people } \\
\text { attending at the } \\
\text { school visit }\end{array}$ & $\begin{array}{l}\text { Children and } \\
\text { adults }\end{array}$ & Open farm, school visit & $\begin{array}{l}\text { C. parvum } \\
\text { (subgenotype } \\
\text { IIaA15G2R1) }\end{array}$ & {$[79]$} \\
\hline Community & Wales (UK) & $\begin{array}{c}36 \text { people } \\
\text { attending at the } \\
\text { visit }\end{array}$ & $\begin{array}{l}\text { Children and } \\
\text { adults }\end{array}$ & $\begin{array}{l}\text { Residential farmcentre, } \\
\text { school visit }\end{array}$ & $\begin{array}{l}\text { C. parvum } \\
\text { C. hominis } \\
\text { C. meleagridis }\end{array}$ & {$[63]$} \\
\hline Waterborne & $\begin{array}{l}\text { Majorca } \\
\text { (Spain) }\end{array}$ & 179 travellers & $\begin{array}{l}\text { Children and } \\
\text { adults }\end{array}$ & $\begin{array}{l}\text { Hotel pool water } \\
\text { contamination }\end{array}$ & $\begin{array}{l}\text { Cryptosporidium } \\
\text { spp. }\end{array}$ & {$[292]$} \\
\hline Foodborne & $\mathrm{OH}$ (USA) & 144 inhabitants & $\begin{array}{l}\text { Children and } \\
\text { adults }\end{array}$ & $\begin{array}{l}\text { Contamination of } \\
\text { unpasteurized apple } \\
\text { cider }\end{array}$ & $\begin{array}{c}\text { C. parvum } \\
\text { subgenotype } \\
\text { IIaA15G2R1, } \\
\text { IIaA17G2R1 }\end{array}$ & {$[182]$} \\
\hline Community & MN (USA) & $\begin{array}{l}31 \text { middle-/high- } \\
\text { school } \\
\text { students }\end{array}$ & Young people & Contact with calves & C. parvum & {$[303]$} \\
\hline Community & MN (USA) & $\begin{array}{l}37 \text { middle-/high- } \\
\text { school } \\
\text { students }\end{array}$ & Young people & Manure on hands & C. parvum & {$[303]$} \\
\hline Foodborne & MN (USA) & 9 people & Not reported & $\begin{array}{l}\text { Contamination of food } \\
\text { in a hotel banquet room }\end{array}$ & $\begin{array}{l}\text { Cryptosporidium } \\
\text { spp. }\end{array}$ & $\begin{array}{l}\text { http://www.cdc.gov/ } \\
\text { foodborneoutbreaks/ } \\
\text { outbreak_data.htm }\end{array}$ \\
\hline
\end{tabular}


Table 4: Continued.

\begin{tabular}{|c|c|c|c|c|c|c|}
\hline $\begin{array}{l}\text { Outbreak }^{1} \\
\text { type }\end{array}$ & Country & $\mathrm{Ill}^{2}$ & Age & $\begin{array}{c}\text { Likely causes for } \\
\text { outbreak occurring }\end{array}$ & $\begin{array}{c}\text { Species } \\
\text { /genotype }\end{array}$ & Reference \\
\hline \multicolumn{7}{|c|}{2002} \\
\hline Community & $\begin{array}{l}\text { Yorkshire and } \\
\text { The Humber } \\
\text { (UK) }\end{array}$ & $\begin{array}{c}47 \text { people } \\
\text { attending at the } \\
\text { nursery }\end{array}$ & $\begin{array}{l}\text { Children and } \\
\text { adults }\end{array}$ & $\begin{array}{l}\text { Contamination at a day } \\
\text { care nursery }\end{array}$ & $\begin{array}{l}\text { C. parvum } \\
\text { C. hominis } \\
\text { C. meleagridis }\end{array}$ & {$[63]$} \\
\hline $\begin{array}{l}\text { Multiple } \\
\text { exposure: } \\
\text { Waterborne } \\
\text { and } \\
\text { Community }\end{array}$ & Wales (UK) & 4 people & $\begin{array}{l}3 \text { children and } 1 \\
\text { adult }\end{array}$ & $\begin{array}{l}\text { Contamination of a } \\
\text { private drinking water } \\
\text { supply private water } \\
\text { supply, farm visits and } \\
\text { personal contact }\end{array}$ & $\begin{array}{c}\text { C. parvum } \\
\text { (subgenotype } \\
\text { IIaA17G1R1) }\end{array}$ & {$[79]$} \\
\hline Waterborne & $\begin{array}{l}\text { South East of } \\
\text { England } \\
\text { (UK) }\end{array}$ & 21 people & Not reported & $\begin{array}{l}\text { Contamination of a } \\
\text { public drinking water } \\
\text { supply }\end{array}$ & $\begin{array}{l}\text { Cryptosporidium } \\
\text { spp. }\end{array}$ & [293] \\
\hline Waterborne & $\begin{array}{l}\text { South East of } \\
\text { England } \\
\text { (UK) }\end{array}$ & 31 people & Not reported & $\begin{array}{l}\text { Contamination of a } \\
\text { public drinking water } \\
\text { supply }\end{array}$ & $\begin{array}{l}\text { Cryptosporidium } \\
\text { spp. }\end{array}$ & [293] \\
\hline Waterborne & $\begin{array}{l}\text { North West } \\
\text { of England } \\
\text { (UK) }\end{array}$ & 50 school people $^{8}$ & $\begin{array}{l}\text { Adults and } \\
\text { children }\end{array}$ & $\begin{array}{l}\text { Contamination of a } \\
\text { private drinking water } \\
\text { supply at a college }\end{array}$ & $\begin{array}{l}\text { Cryptosporidium } \\
\text { spp. }\end{array}$ & {$[293,294]$} \\
\hline Waterborne & $\begin{array}{l}\text { Northern } \\
\text { Ireland (UK) }\end{array}$ & 29 people & Adults & $\begin{array}{c}\text { Contamination of raw } \\
\text { and treated water, and } \\
\text { land surrounding the } \\
\text { lake watershed }\end{array}$ & $\begin{array}{l}\text { Cryptosporidium } \\
\text { spp. }\end{array}$ & [99] \\
\hline Community & Netherlands & Not reported & Children & $\begin{array}{l}\text { Not reported, during a } \\
\text { pet farm visit }\end{array}$ & $\begin{array}{c}\text { C. parvum } \\
\text { (genotype } \mathrm{C} 1 \text { ) }\end{array}$ & $\begin{array}{l}\text { http://www.crypto- } \\
\text { sporidium.it/ }\end{array}$ \\
\hline Community & NY (USA) & $\begin{array}{l}13 \text { veterinary } \\
\text { students }\end{array}$ & Young people & $\begin{array}{l}\text { Hands contamination by } \\
\text { calves contacts }\end{array}$ & $\begin{array}{l}\text { Cryptosporidium } \\
\text { spp. }\end{array}$ & {$[304]$} \\
\hline Foodborne & FL (USA) & 37 people & Not reported & $\begin{array}{l}\text { Contamination of food } \\
\text { in a hotel banquet room }\end{array}$ & $\begin{array}{l}\text { Cryptosporidium } \\
\text { spp. }\end{array}$ & $\begin{array}{l}\text { http://www.cdc.gov/ } \\
\text { foodborneoutbreaks/ }\end{array}$ \\
\hline Foodborne & GA (USA) & 6 people & Not reported & $\begin{array}{l}\text { Contamination of food } \\
\text { in a private home }\end{array}$ & $\begin{array}{l}\text { Cryptosporidium } \\
\text { spp. }\end{array}$ & outbreak_data.htm \\
\hline \multicolumn{7}{|c|}{2001} \\
\hline Waterborne & France & $\begin{array}{l}291 \text { county } \\
\text { inhabitants }\end{array}$ & $\begin{array}{l}\text { Adults and } \\
\text { children }\end{array}$ & $\begin{array}{l}\text { Public water supply } \\
\text { contamination }\end{array}$ & $\begin{array}{c}\text { C. hominis } \\
\text { (genotype Ib,Id) } \\
\text { C. parvum } \\
\text { (genotype IIa) }\end{array}$ & {$[84]$} \\
\hline Waterborne & France & 573 & Adults & $\begin{array}{l}\text { Contamination of tap } \\
\text { water }\end{array}$ & $\begin{array}{l}\text { C. parvum } \\
\text { (genotype } 1 \text { ) }\end{array}$ & {$[305]$} \\
\hline Waterborne & $\begin{array}{l}\text { South West of } \\
\text { England } \\
\text { (UK) }\end{array}$ & 14 & $\begin{array}{l}\text { Adults and } \\
\text { children }\end{array}$ & $\begin{array}{c}\text { Contact with astream at } \\
\text { a beach }\end{array}$ & $\begin{array}{l}\text { Cryptosporidium } \\
\text { spp. }\end{array}$ & [294] \\
\hline Community & $\begin{array}{l}\text { South East of } \\
\text { England } \\
\text { (UK) }\end{array}$ & 30 & $\begin{array}{l}\text { Adults and } \\
\text { children }\end{array}$ & $\begin{array}{l}\text { Contamination at a day } \\
\text { care nursery }\end{array}$ & $\begin{array}{l}\text { Cryptosporidium } \\
\text { spp. }\end{array}$ & Unpublished data \\
\hline Waterborne & $\begin{array}{l}\text { South East of } \\
\text { England } \\
\text { (UK) }\end{array}$ & $\begin{array}{l}152 \text { people } \\
\text { attending at a } \\
\text { school }^{8}\end{array}$ & $\begin{array}{l}\text { Adults and } \\
\text { children }\end{array}$ & $\begin{array}{c}\text { Contamination of } \\
\text { outdoor school pool } \\
\text { water }\end{array}$ & $\begin{array}{l}\text { Cryptosporidium } \\
\text { spp. }\end{array}$ & {$[294,295]$} \\
\hline Waterborne & $\begin{array}{l}\text { Canada } \\
\text { (USA) }\end{array}$ & 1039 people & Young adults & $\begin{array}{l}\text { Contamination of } \\
\text { drinking water }\end{array}$ & C. parvum & [296] \\
\hline Waterborne & $\begin{array}{l}\text { Canada } \\
\text { (USA) }\end{array}$ & $\begin{array}{c}59 \text { people } \\
\text { attending an } \\
\text { Ukrainian dance } \\
\text { festival }\end{array}$ & Adults & $\begin{array}{l}\text { Contamination of a } \\
\text { swimming pool in a } \\
\text { hotel }\end{array}$ & C. parvum & [297] \\
\hline Waterborne & (IL) USA & $\begin{array}{l}358 \text { waterpark } \\
\text { attendants }\end{array}$ & $\begin{array}{l}\text { Adults and } \\
\text { children }\end{array}$ & $\begin{array}{l}\text { Contamination of } \\
\text { waterpark and } \\
\text { person-to-person } \\
\text { contact }\end{array}$ & $\begin{array}{l}\text { C. hominis } \\
\text { (genotype Ia) }\end{array}$ & [259] \\
\hline
\end{tabular}


TABle 4: Continued.

\begin{tabular}{|c|c|c|c|c|c|c|}
\hline $\begin{array}{l}\text { Outbreak }^{1} \\
\text { type }\end{array}$ & Country & $\mathrm{Ill}^{2}$ & Age & $\begin{array}{l}\text { Likely causes for } \\
\text { outbreak occurring }\end{array}$ & $\begin{array}{c}\text { Species } \\
\text { /genotype }\end{array}$ & Reference \\
\hline Foodborne & $\begin{array}{l}\text { Queensland } \\
\text { (Australia) }\end{array}$ & 8 inhabitants & Children & $\begin{array}{c}\text { Contamination of } \\
\text { drinking unpasteurised } \\
\text { milk }\end{array}$ & $\begin{array}{l}\text { Cryptosporidium } \\
\text { spp. }\end{array}$ & {$[262]$} \\
\hline Community & New Zealand & $\begin{array}{c}20 \text { farm } \\
\text { inhabitants }\end{array}$ & Children & $\begin{array}{l}\text { Hand contamination by } \\
\text { calve contact }\end{array}$ & $\begin{array}{l}\text { Cryptosporidium } \\
\text { spp. }\end{array}$ & {$[264]$} \\
\hline Community & $\begin{array}{l}\text { Tasmania } \\
\text { (Australia) }\end{array}$ & $\begin{array}{c}36 \text { participants at } \\
\text { the agricultural } \\
\text { show }\end{array}$ & Adults & $\begin{array}{c}\text { Contamination } \\
\text { associated with an } \\
\text { animal nursery }\end{array}$ & $\begin{array}{l}\text { Cryptosporidium } \\
\text { spp. }\end{array}$ & {$[306]$} \\
\hline Community & Brazil & $\begin{array}{c}224 \text { day care } \\
\text { attendants }\end{array}$ & Children & $\begin{array}{l}\text { Person-to-person } \\
\text { contact }\end{array}$ & $\begin{array}{l}\text { C. hominis } \\
\text { (genotype 1) }\end{array}$ & {$[307]$} \\
\hline \multicolumn{7}{|c|}{$2001-2000$} \\
\hline Waterborne & $\begin{array}{l}\text { Northern } \\
\text { Ireland (UK) }\end{array}$ & 347 & Adults & $\begin{array}{l}\text { Contamination of } \\
\text { drinking water }\end{array}$ & $\begin{array}{l}\text { C. parvum } \\
\text { (bovine } \\
\text { genotype; } \\
\text { human } \\
\text { genotype) }\end{array}$ & {$[80]$} \\
\hline Waterborne & Haiti & 93 patients & $\begin{array}{l}\text { Adults and } \\
\text { children }\end{array}$ & $\begin{array}{c}\text { Contaminated water and } \\
\text { overcrowded conditions } \\
\text { of urban slums }\end{array}$ & $\begin{array}{l}\text { C. hominis, } \\
\text { C. parvum, } \\
\text { C. felis } \\
\end{array}$ & {$[69]$} \\
\hline \multicolumn{7}{|c|}{2000} \\
\hline Waterborne & $\begin{array}{l}\text { England and } \\
\text { Wales (UK) }\end{array}$ & 58 & Adults & $\begin{array}{l}\text { Contamination of } \\
\text { drinking water }\end{array}$ & $\begin{array}{c}\text { C. parvum } \\
\text { (genotype 2) }\end{array}$ & {$[308]$} \\
\hline Waterborne & $\begin{array}{l}\text { England and } \\
\text { Wales (UK) }\end{array}$ & 207 & Not reported & $\begin{array}{l}\text { Contamination of } \\
\text { drinking water }\end{array}$ & $\begin{array}{l}\text { C. hominis and } \\
\text { C. parvum } \\
\text { alleles }\end{array}$ & [309] \\
\hline Waterborne & $\begin{array}{l}\text { Yorkshire and } \\
\text { The Humber } \\
\text { (UK) }\end{array}$ & $\begin{array}{c}41 \text { people } \\
\text { attending a public } \\
\text { pool }\end{array}$ & $\begin{array}{l}\text { Adults and } \\
\text { children }\end{array}$ & $\begin{array}{l}\text { Contamination of pool } \\
\text { water }\end{array}$ & $\begin{array}{c}\text { C. parvum } \\
\text { (subgenotype } \\
\text { IIaA17G1R1) }\end{array}$ & {$[79,295]$} \\
\hline Waterborne & $\begin{array}{l}\text { Majorca } \\
\text { (Spain) }\end{array}$ & $>250$ & $\begin{array}{l}\text { Adults and } \\
\text { children }\end{array}$ & $\begin{array}{l}\text { Contamination of a } \\
\text { hotel pool water }\end{array}$ & $\begin{array}{l}\text { Cryptosporidium } \\
\text { spp. }\end{array}$ & {$[81]$} \\
\hline Community & Netherlands & Not reported & Children & $\begin{array}{l}\text { School children visiting } \\
\text { a pet farm }\end{array}$ & $\begin{array}{l}\text { Cryptosporidium } \\
\text { spp. }\end{array}$ & $\begin{array}{l}\text { http://www.crypto- } \\
\text { sporidium.it/ }\end{array}$ \\
\hline Foodborne & IL (USA) & 8 & Not reported & $\begin{array}{c}\text { Contamination of } \\
\text { coleslaw in a private } \\
\text { home }\end{array}$ & C. parvum & $\begin{array}{l}\text { http://www.cdc.gov/ } \\
\text { foodborneoutbreaks/ } \\
\text { outbreak_data.htm }\end{array}$ \\
\hline \multicolumn{7}{|c|}{1999} \\
\hline Waterborne & Russia & 50 & Adults & $\begin{array}{l}\text { Contamination of } \\
\text { drinking water }\end{array}$ & $\begin{array}{l}\text { Cryptosporidium } \\
\text { spp. }\end{array}$ & {$[310]$} \\
\hline Waterborne & FL (USA) & 38 park visitors & $\begin{array}{l}\text { Adults and } \\
\text { children }\end{array}$ & $\begin{array}{c}\text { Contamination of a } \\
\text { water fountain }\end{array}$ & $\begin{array}{l}\text { Cryptosporidium } \\
\text { spp. }\end{array}$ & {$[260]$} \\
\hline \multicolumn{7}{|c|}{1998} \\
\hline Waterborne & Spain & 21 & Children & $\begin{array}{c}\text { Contamination of tap } \\
\text { water }\end{array}$ & $\begin{array}{l}\text { Cryptosporidium } \\
\text { spp. }\end{array}$ & {$[253]$} \\
\hline Foodborne & DC (USA) & $\begin{array}{l}88 \text { students and } \\
\text { employees }\end{array}$ & $\begin{array}{l}\text { Young adults } \\
\text { and adults }\end{array}$ & $\begin{array}{c}\text { Contamination of food } \\
\text { by a food handler in a } \\
\text { cafeteria }\end{array}$ & $\begin{array}{l}\text { Cryptosporidium } \\
\text { spp. }\end{array}$ & {$[311]$} \\
\hline
\end{tabular}

${ }^{1}$ In presence of two or more cases of similar infection, with a common exposure in the community not related to waterborne or foodborne diasese, the term of community disease was used.

${ }^{2}$ Number and category of people with symptom referable to cryptosporidiosis.

${ }^{3}$ This outbreak was characterised by a Cryptosporidium infection in 9 of the 11 children, a coinfection of Giardia and Cryptosporidium in 2 of the 11 children and a concomitant infection of other 38 additional children by only Giardia oocysts triggered by the same likely source.

${ }^{4}$ Median age.

${ }^{5}$ Outbreak characterised by a concomitant waterborne Cyclospora outbreak.

${ }^{6}$ Outbreak characterised by a concomitant waterborne Giardia outbreak.

${ }^{7}$ Outbreak characterised by a concomitant foodborne E. coli O111 outbreak.

${ }^{8}$ Outbreak characterised by a concomitant waterborne Norovirus outbreak. 
in raw and drinking water while other no well-standardised procedures are available for wastewater [109]. However, raw, reclaimed and drinking water are not subjected to routine monitoring. In surface waters (rivers, watersheds, watercourses and lakes), several studies have reported high contaminations by (oo)cysts of Giardia and Cryptosporidium all over the peninsula with the highest prevalence for Giardia [111-114]. Presence of both Cryptosporidium and Giardia has been monitored in sewage, surface waters, drinking water, and swimming pools by simultaneous tracing of bacterial indicators. In the Latium and Apulia regions, cysts and oocysts were detected in sewage and surface water, with Giardia numbers always prevailing over Cryptosporidium, but not in drinking waters. However, remarkably, Cryptosporidium was detected in $9 \%$ of samples collected from swimming pools in the Latium [112]. The paper by Di Benedetto et al., 2005 [113] described the occurrence of Cryptosporidium and Giardia (oo)cysts in water samples of two municipal treatment plants, and in surface and ground water wells in Sicily. The wastewater samples taken before and after treatment process were assayed over the course of one year: Giardia cysts were detected in all samples throught the year at higher concentration levels than Cryptosporidium oocysts, subjected to a peak during Spring. Cysts were detected in one lake at very low concentration; on the contrary, both parasites were found at high-concentration levels in all samples collected through one year from the river waters. The pattern of occurrence of both parasites showed temporal-related relationship to rainfall trend (Table 1) (Figure 3). In the Tuscan area, five drinking water treatment plants, differing for the employed handling, were monitored for the presence of Cryptosporidium and Giardia (oo)cysts, to estimate the removal capacity of each plant [114]. Water samples (from inflow raw water and outflow drinking water) were analysed during a one-year survey and both protozoa were detected. The occurrence of (oo)cysts was not associated with seasonality, turbidity or C. perfringens, but however low performance of plants was correlated with presence of protozoa in outflow drinking waters. Giardia cyst and Cryptosporidium oocyst removal efficiency was also evaluated in a wastewater tertiary treatment system based on membrane ultrafiltration and fed with secondarytreated municipal wastewater in Apulia: G. duodenalis and C. parvum were identified in feed water but were found in filtered water only during occasional failure of the filtration system [115]. Also in raw sewage and primary effluent more Giardia cysts than Cryptosporidium oocysts were detected in many monitoring studies [112, 113, 116, 117].

Annual rainfall reduction in some regions and increased human consumption have caused a shortage of water resources at global level. The recycling of treated wastewaters has been therefore suggested for domestic, industrial, and agricultural activities. Giardia and Cryptosporidium are known to be highly resistant to water treatment procedures and to cause outbreaks through contaminated raw or treated water. The study by Cacciò et al., 2003 [117] performed an investigation in four wastewater treatment plants in Lombardia, Campania, Sardinia, Sicily by sampling wastewater at each stage of the treatment process over the course of one year, and testing the presence of both parasites. While Cryptosporidium oocysts were rarely observed, Giardia cysts were detected in all samples throughout the year, with peaks observed in Autumn and Winter. The massive amounts of feces from humans and animals are discharged, dumped, or carried in runoff, bringing encysted zoonotic protozoan parasites to estuaries and coastal waters, where they contaminate bathing beaches, and are finally filtered and concentrated by shellfish eaten by humans and marine mammals, and infect a wide range of marine animal hosts, resulting in morbidity and mortality to some populations [118]. Therefore, nearshore marine sites may be considered at higher risk for exposure to livestock runoff, human sewage, or both fecal sources. Bivalves filter large volumes of water and can concentrate organisms which are pathogenic for humans and animals. In a recent paper [119] the presence of Cryptosporidium spp. in clams (Chamelea gallina) from the Adriatic coast (Abruzzo) was reported for the first time. The temporal occurrence of Cryptosporidium (C. hominis and C. parvum) oocysts in Ruditapes philippinarum were evaluated in two farms located in Veneto and in Friuli Venezia Giulia [120]. The paper of Giangaspero et al., 2009 [121], has simultaneously investigated the presence of Giardia and Cryptosporidium in inflowing water and harvested shellfish (Ruditapes decussatus and Mytilus galloprovincialis) in geographically closed environment (Varano Lagoon, Apulia). Higher concentrations of Giardia cysts than Cryptosporidium oocysts were registered in almost all wastewater and water samples, but testing of shellfish gave negative results for both protozoa. However, Cryptosporidium (C. parvum, C. felis, C. andersoni, and two novel genotypes) was detected in haemolymph samples from mussels in California [122]. In this paper, factors significantly associated with detection of Cryptosporidium spp. in mussel batches were exposure to freshwater outflow and collection within a week following a precipitation event, while no correlation was found with exposure to livestock feces or human sewage sources. Remarkably, mussels were proposed as tracer to monitor water quality, suggesting that humans and animals ingesting shellfish may be exposed to both hostspecific and anthropozoonotic Cryptosporidium genotypes of public health significance [122].

In the last decade, a major concern for the scientific community has been whether infected animals can serve as reservoirs of Giardia and Cryptosporidium infection for humans. The paper by Giangaspero et al., 2007 [109], presented data on prevalence and molecular genotyping from several sample types (companion animals, sheep, cattle, goats, wastewaters, surface water, shellfish, and humans) collected in the Italian territory. Several species/genotypes of Cryptosporidium have a relevant zoonotic potential and ruminants may be important sources of infection for human beings [106, 108]. Cryptosporidiosis causes important economic losses to animal husbandry and livestock production. To obtain information on the occurrence of cryptosporidiosis in lambs and the potential zoonotic role of the Cryptosporidium isolates, fecal samples collected from lambs in Central Italy (Abruzzo) were examined for the presence of Cryptosporidium and discussed in the paper 
by Paoletti et al., 2009 [123]. All positive samples were characterised as zoonotic C. parvum genotype suggesting a potential public health hazard in Italy. Also the risk related to bovine zoonotic contribution was studied by Duranti et al., 2009 [124] by considering 248 farms in Central Italy (Latium and Marche). In all positive samples, the etiological agent was identified as $C$. parvum with a large subtype genetic variability. The prevalence of farm infection ranged from $3.4 \%$ to $35.6 \%$ and appeared related to putative risk factors such as farm type, calve stalling, late supply of colostrum, number of heads and contact between calves and adults. However, the highest risk was associated with housing calves separately from their dams, whereas dam nursing resulted as a protective factor. This important evidence consistently agrees with the role of maternal milk as protective factor against cryptosporidiosis onset both in animals [125] and in humans [126].

3.3. Waterborne Cryptosporidosis in USA and Canada. Besides zoonotic transmission, an important identified waterborne infection route is linked to recreational waters [6]. A cryptosporidiosis outbreak from August to September 2004 in California, affected more than 250 people visiting a waterpark [127] (Table 4). Occurring more than a decade after the first reported outbreak of cryptosporidiosis in a swimming pool [128], this outbreak demonstrates that recreational waters may represent a highly potential infection vehicle especially in childhood. Recently in USA Cryptosporidium species have emerged as a major cause of outbreaks of diarrhoea [6] and have been associated with consumption of contaminated recreational and drinking waters and with the attendance to child-care programmes [129]. Principal risk factors for infection seem to coincide with swallowing untreated water from a lake, river, or after exposure to recreational water or, remarkably, after contact with a child in a child-care programme or with diapers [129] (see Section 7). Although exposure to recreational water is commonly implicated in summertime cryptosporidiosis outbreaks, this evidence demonstrates that investigations of increased incidence of cases in Summer should also examine other potential risk factors, addressing the multiple transmission routes for Cryptosporidium. The extended review of Craun et al. 2005 [130], on outbreaks associated with recreational water during 1971-2000 in USA, provided evidence that bacterial or protozoan etiology was identified in three-quarters of the outbreaks. Outbreaks caused by Cryptosporidium and Giardia were primarily associated with treated water in swimming and wading pools. Contamination from sewage discharges and wild or domestic animals were also recognized as important sources for untreated waters. Contributing factors in swimmingpool outbreaks were inadequate attention to maintenance, operation, disinfection, and filtration [131]. For the 764 waterborne outbreaks registered from 1971 to 2002 by the USA National Surveillance of outbreaks and associated with drinking water, 575457 cases of illness and 79 deaths were reported [132, 133]. If properly applied, current protocols in municipal water treatment are effective at eliminating pathogens from water. However, inadequate, interrupted, or intermittent treatment has repeatedly been associated with incidence of waterborne disease outbreaks. Contamination is affected by the number of pathogens in the source water, the age of the distribution system, the quality of the delivered water, and climatic events that can tax treatment plant operations. Furthermore, private water supplies are not regulated by the USEPA and are generally not treated or monitored [134].

A case-control study [135] was conducted in the San Francisco Bay Area as part of a national study sponsored by the CDC [136] (Table 3) to ascertain the major routes of transmission for endemic cryptosporidiosis, with an emphasis on evaluating risk from drinking water. Drinking and recreational water, food items, travel, animal contact, person-to-person fecal contact, and (for adults) sexual practices were evaluated as major exposures. The study showed no significant association between cryptosporidiosis and drinking water among the immunocompetent population in the San Francisco Bay, and therefore, the key risk factor for cryptosporidiosis in this area was identified in travelling to another country [135].

The recent paper by Reynolds et al., 2008 [137] has provided estimates of waterborne infection and illness risks in the USA by considering the correlation between the total number of water systems, source water type, total populations exposed, and microbial infection. The results indicated $10.7 \times 10^{6}$ and $2.2 \times 10^{6}$ infections/year in populations served by community and noncommunity groundwater systems, respectively, and $26.0 \times 10^{6}$ infections/year in populations with municipal surface water system services. Water purification technologies applied at the point-ofuse (POU) could be effective for limiting the effects of source water contamination, treatment plant inadequacies, minor intrusions in the distribution system, or deliberate posttreatment acts as bioterrorism (i.e. Cryptosporidium is a Category B bioterrorist threat) [93]. However, epidemiological studies are conflicting on the benefits of POU water treatment compared to untreated tap water [138141]. Nevertheless, for immunocompromised and other populations, including those experiencing physiological life stages such as pregnancy, or those very young or very old, POU devices may represent water treatment options for reducing risks of Cryptosporidium and other types of infectious agents transmitted by drinking water. A study [142], aimed to estimate the urban contribution to the total Cryptosporidium and Giardia receiving-water loads in USA, was focused on combined sewer overflows (CSO), discharges of mixed untreated sewage and stormwaters. Interestingly, CSO from urban areas was not found to be a significant contributor of Cryptosporidium, but a significative source of Giardia [142]. Most cryptosporidiosis outbreaks in the USA are caused by C. hominis, and this species is often reported as the primary cause of cryptosporidiosis in this country $[143,144]$. However, outbreaks account for only $10 \%$ of the overall cryptosporidiosis cases, and there are still few data on the species causing sporadic cases [145] (Table 2). The highest incidence of cryptosporidiosis in the USA has been found in the upper Midwest States [146]. In particular, Wisconsin was reported as having the highest 
incidence of cryptosporidiosis every year from 1999 to 2002 [146]. The Wisconsin city of Milwaukee also had the largest cryptosporidiosis outbreak in 1993, where more than 400000 people were infected following contamination of the municipal water supply [147]. The pivotal study by Feltus et al., 2006 [148], identified for 49 cases of sporadic cryptosporidiosis in Wisconsin, during the period from 2003 to 2005, C. parvum, C. hominis, a cervine genotype [64], a cervine genotype variant, and a new W17 human genotypes [149] (Table 2). However, the study showed that most cases were linked to zoonotic Cryptosporidium genotypes. The Tri-County Health Department of Colorado [150] (Table 3) investigated an outbreak of cryptosporidiosis occurred in 2006, linked to a community swimming pool treated by chlorination and UV light irradiation. Risk factors appeared through swimming, getting water in mouth, and swallowing water. Important studies on waterbornetransmitted infections have been also conducted in Canada. In South Western Ontario, from July 2002 to December 2003, water samples were collected from 36 locations within the Grand River Watershed, and were analyzed for total coliforms, fecal coliforms, E. coli, E. coli O157:H7, thermophilic Campylobacter spp., culturable human enteric viruses, C. perfringens, Cryptosporidium spp., and Giardia spp. [151]. Peaks in pathogen numbers frequently preceded the peaks in numbers and turbidity of indicator organisms suggesting important implications (e.g., pathogen transport model) for designing monitoring programs in source water risk assessment [151]. In Southern Ontario, to identify management practices associated with an increased withinherd prevalence of $C$. parvum shedding on dairy farms, a large study was conducted on fecal samples collected from 1089 calves in 119 herds [125]. Overall, 30\% of the calves were shedding $C$. parvum oocysts, with a prevalence ranged from 0 to $80 \%$ within herds and at least one positive calf detected in $77 \%$ of herds. Predictors significantly associated with an increased prevalence of shedding were the use of calf rub prophylaxis in cows and the feeding by milk replacer in the first week of life. In contrast, the presence of concrete flooring in calf housing areas and the use of soap or detergent when washing calf feeding utensils appeared to be protective [125].

The QMRA model was recently applied to assess the relative risks of infection associated with the presence of Cryptosporidium and Giardia in drinking water in Canada [152]. The assessment of the final risk in the contamination of the water plants resulted considerably affected by the selection of treatment performance model (filtration and ozonation). Recently, data from a sentinel site (Waterloo Region, Ontario) of the C-EnterNet (Table 3) [153, 154] were used to assess exposure factors on laboratory-confirmed Cryptosporidium infections [155]. Of 1204 cases of enteric illness in the sentinel area between April 2005 and December 2007, 36 cases were selected after excluding outbreak and international travel-related cases. Cryptosporidiosis was associated with swimming in a lake or river, drinking municipal water, and having a family member with a diarrhoeal illness. Since 1971, the Waterborne Disease and Outbreak Surveillance System has reported on waterborne disease and outbreak (WBDO)-related data. In 1978, WBDOs associated with recreational waters (natural and treated waters) were added [156]. During 2003-2004, a total of $62 \mathrm{WBDOs}$ associated with recreational water were reported by 27 states, with typical illness occurred in 2,698 persons, resulting in 58 hospitalizations and one death [156]. Of the 62 WBDOs, 30 were outbreaks of gastroenteritis and Cryptosporidium was confirmed as the causal agent in 11, and all except one of these outbreaks occurred in treated water venues [156] (Table 4). Lastly, approximately 90\% of waterborne outbreaks occur in treated recreational waters (swimming pools, spas and recreational parks), while the remaining $10 \%$ arise from natural waters used for leisure (e.g., bathing in rivers, beaches, etc) [6].

\subsection{Waterborne Cryptosporidiosis in New Zealand and Aus-} tralia. New Zealand has a higher incidence of cryptosporidiosis compared to other developed countries. A recent study in [157] aimed to thoroughly describe the epidemiology of this disease and to identify specific potential risk factors by analysing anonymous cryptosporidiosis notification and hospitalisation data. Human cases were designated as "urban" or "rural" and an association between disease rates and animal density was studied. Over the 10-year period from 1997 to 2006, the average annual rate of notified cryptosporidiosis was 22 cases per 100000 population. The number of hospitalisations amounted to $3.6 \%$ of the notified cases. The annual incidence of infection appeared fairly stable, but showed marked seasonality with a peak rate in Spring. The highest rates were among Europeans, children 0-9 years of age, and those living in low-deprivation areas (Table 1) (Figure 3). Notification rates showed large geographic variations, with rates in rural areas 2.8 times higher than in urban areas, and with rural areas also experiencing the most pronounced Spring peak, correlated with farm animal density. Therefore, most transmission of Cryptosporidium in New Zealand appears to be zoonotic in rural settings [157]. These data seem to corroborate the evidence that the proportion of $C$. parvum sporadic cases in humans is higher in rural than in urban areas [158] and confirm the variation of the geographical distribution of Cryptosporidium infections within countries [159]. In New South Wales (NSW, Australia) the subtypes have global distributions and indicate both anthroponotic and zoonotic transmission routes in sporadic cryptosporidiosis [160].

\subsection{Waterborne Cryptosporidiosis in African Developing} Countries. The burden of disease from cryptosporidiosis in developing countries is in the Sub-Saharan Africa, because of the disseminated status of malnutrition in children and the highest world prevalence of HIV infection in this region, with peaks reaching the $15 \%-28 \%$ of the adult population [161] (Table 2). However, in this vast geographical area, Cryptosporidium and Giardia infections are rampant also in adult and immunocompetent populations, due to the unhygienic and improper disposal of wastewater and to the use of surface waters as major source of potable water. The important research by Gideon et al. 2007 [162], represents 
a preliminary attempt to isolate, identify, and quantify recurrent forms of these emerging protozoa in the waste and surface waters in Cameroon. The cyst and oocyst counts were higher in the direct effluent because they were released directly into the wastewater by feces, while they were diluted in the surface water, reducing their abundance [162]. Clearly the nonfunctional water purification stations and ineffective water treatment systems expose the community to potential outbreaks; however, surveillance plans are still completely lacking. In North Africa cryptosporidiosis is prevailingly monitored as public health issue and, recently, a comprehensive review of $C$. parvum epidemiology has been produced with the aim to describe the burden associated with cryptosporidiosis among Egypt and close countries [163]. C. parvum prevalence ranged from $0 \%-47 \%$. Remarkably, identified risk factors, including population, ecology, and environmental findings, suggested water and zoonotic transmission modalities as the principal route of infection [163]. A recent paper [164] has provided evidence on the link between diarrheal diseases of humans and young calves in the Middle East (Iran). By exploiting genotyping approach, the authors have inferred both anthroponotic and zoonotic transmission of cryptosporidiosis in this geographical area [164] (Table 2).

3.6. Waterborne Cryptosporidiosis in Asia. In Asia, the emerging need to facilitate the characterization of the endemic transmission of cryptosporidiosis has recently provided a large study [41] on genotype distributions of Cryptosporidium oocysts in domestic wastewater in China (Table 2). Raw domestic wastewater samples were collected from four wastewater treatment plants in Shanghai, from December 2006 to April 2007. Interestingly, diverse Cryptosporidium species/genotypes were identified and $C$. hominis subtyping revealed a high complexity of Cryptosporidium populations often unique (Table 2) (see Introduction, Section 1.6).

The specific contribution of the zoonotic transmission of Cryptosporidium in China was discussed in the recent paper by Liu et al., 2009 [165], in which a total of 507 fecal specimens from six dairy farms were examined for Cryptosporidium spp. Interestingly were identified C. andersoni and Cryptosporidium ryanae [166], a new species described from cattle and previously identified as the Cryptosporidium deer-like genotype [167], with $C$. andersoni as the dominant species. This interesting distribution of Cryptosporidium spp. may support the idea of unique species and transmission in these areas. In Taiwan, cryptosporidia were detected in most of the surface water specimens [168]. Water samples collected from potable water treatment plants were investigated for the presence of Giardia cysts and Cryptosporidium oocysts. The frequency of occurrence of (oo)cysts was $78 \%$ for Giardia and $72 \%$ for Cryptosporidium in 18 raw water samples. Ten out of 13 samples collected from treated water samples showed the presence of cysts, while oocysts were detected in five out of 13 treated water samples. The risk assessment for the presence of cysts and oocysts, indicates the possibility of waterborne transmission of Giardia and Cryptosporidium infection in Taiwan where adequate water treatment is almost absent. In India, an important work [169] reported on the correlation between infections in livestock and seasonal rainfall by considering the monsoon period impact on cryptosporidiosis. The survey revealed a $30 \%$ infection with C. parvum, out of 457 fecal samples collected from neonatal bovine calves (0-3 months of age) from dairy farms for one year, across three different geographical and agro-climatic areas of India (Northern subtemperate, Eastern subtropical, and Southern subtropical region), and through the premonsoon, monsoon and postmonsoon periods. The infection was more prevalent in the Northern parts of the country than in the Eastern or Southern areas, and C. parvum was detected as the only species [169]. Highest prevalence was recorded during monsoon months. A more recent study [170], however, performed on 350 fecal samples collected from juvenile and mature cattle (6-24 months of age), across the three representative agro-climatic regions of the country, showed the only presence of $C$. andersoni with the highest occurrence in the Northern states [170]. The animals between age group of 6-12 months were mostly affected and the seasonal prevalence was higher during the hot and humid monsoon season, followed by the premonsoon season when the climate is hot and humid. However, consistently with Paul et al., 2008 [169], in the post-monsoon season the prevalence dropped, providing evidence for transmission related to survivability of the infective stages of the parasite [46]. The results seemed to suggest that $C$. andersoni is the major Cryptosporidium species affecting cattle with the increase in age, despite differences in species may also be associated to geography differences in sampling. However, unlike previous studies $[169,171]$, no cases of C. parvum, C. bovis and Cryptosporidium deer-like genotype [167] were found in the report of Paul et al., 2009 [170], evidence which may be ascribed to specific prevalence pattern of $C$. andersoni in the areas and season undertaken for study, corroborating the idea of space and time frames for transmission [36]. The animal age undoubtedly remains the most effective risk index in investigating veterinary transmission of Cryptosporidium [46]. Livestock fecal pollution of water sources appears to be the leading cause for both outbreaks and sporadic cases of cryptosporidiosis in developing countries, as already reported in high-income countries [159, 172, 173].

3.7. Waterborne Cryptosporidiosis in South and Central America. To study the prevalence of Cryptosporidium infection, levels of anti-Cryptosporidium IgG antibodies were measured among people inhabiting neighbourhood of a periurban area in the Northeast of Brazil [174]. The study aimed to investigate the effects of environmental sanitation measures, hygienic habits, and household water supply, storage and handling on the frequency of these antibodies in the population sera, providing a model for low-income countries. Cryptosporidium interhousehold transmission was studied by comparing the frequency of anti-Cryptosporidium IgG antibodies among people inhabiting areas with or without different environmental sanitation measures and intrahousehold transmission by comparing the presence of these 
antibodies in families with or without cases of diarrhoea, associated with the presence of Cryptosporidium oocysts in stools. A statistically significant difference was detected in the prevalence of Cryptosporidium infection between areas without and with environmental sanitation measures. Positive associations were found between poor household water supply, drinking unboiled/unfiltered water and high levels of anti-Cryptosporidium antibodies in sera, suggesting uncorrected household water supply, storage and handling as an important factor on Cryptosporidium transmission in developing countries cities [174].

In Central Mexico, the economy of the country is strongly based on sheep and bovine farm management and preventive veterinary medicine represents a useful approach to identify risk factors for zoonotic transmission. To establish the relationship between sheep farm management practices and cryptosporidiosis in this country, 37 farms were mapped to highlight facility characteristics, cleaning measures, water use and animal management practices [175]. Five indexes showed statistical significance: (i) watering frequency; (ii) bottle cleaning frequency; (iii) forage storage; (iv) place of parturition; (v) grazing place. The latter index provided the most relevant association between management practices and cryptosporidiosis. Grazing place may represent a crucial risk factor for cryptosporidiosis in Mexico, contributing to understanding how domestic animals and wildlife cycles interact, resulting in human infections and endemic locations.

3.8. Conclusions. Advanced molecular tools and improvement of international surveillance networks (Table 3 ) are now beginning to answer epidemiological questions related to waterborne transmission which is still difficult to address by traditional methods. Indeed environmental sampling surveys are often hampered by the absence of proper technologies able to provide reliable water sampling collections. Furthermore, many geographical gaps need to be filled to evaluate worldwide waterborne infection distributions and to assess the relationship between animal, human fecal wastes, and water transmission both in undeveloped and developed countries.

\section{Cryptosporidiosis a "Foodborne Disease"}

4.1. Food-Related Routes. In our analysis of Cryptosporidium-linked outbreaks, 15 out of $71(21.1 \%)$ appear to be correlated to foodborne transmission, with a higher number of outbreak episodes in 2006 and 2008. Geographically, the outbreaks seem to be concentrated in the USA, Canada, and Australia and in North Europe, especially Finland and Sweden (Table 4) (Figure 4). Many infection routes have been identified, as consuming salad vegetables washed by contaminated water, eating raw meals, using contaminated water for making ice and frozen/chilled foods, or making products which receive minimum heat or preservative treatment (Table 4). However, contact with contaminated feces transmitted by coprophagoustransport hosts (e.g., birds and insects), worker aerosols (from sneezes), and exposed hand lesions have also been associated with outbreaks [176]. Transfer of pathogens has been documented through contaminated fabrics and carpets, rings, currency, skin surfaces, dust, and aerosols and though person-to-person transmission [176].

4.2. Foodborne Cryptosporidiosis in Europe. In the 27 member states of the European Union, zoonotic parasites transmitted by food are circulating with different prevalence according to the country, the environmental conditions, the human behaviour, and the socioeconomic level. Foodborne parasites can be divided into two main groups according to human transmission. They reach the human beings through the consumption of raw infected food such as muscle tissues of different animal species (T. gondii, Sarcocystis hominis, Sarcocystis suishominis, Diphyllobotrium latum, Taenia solium, Taenia saginata, Opisthorchis felineus, Anisakis spp., Pseudoterranova spp., Trichinella spp.), or vegetables (Fasciola hepatica), and contaminated food and water resources (G. duodenalis, Cryptosporidium spp., T. gondii, Echinococcus granulosus sensu latu, Echinococcus multilocularis, T. solium, Taenia multiceps) [177]. Remarkably, foodborne outbreaks of cryptosporidiosis are considerably increasing in Northern Europe, as shown by the two important outbreaks registered in Finland and Sweden during 2008 [178, 179] (Table 4) (Figure 4).

In 2005 an outbreak of diarrhoea, affecting a group of 99 company employees, was described near Copenhagen [180] (Table 4). All people were ill and 13 positive for C. hominis infection. Disease was associated with eating from the canteen salad bar on one, possibly two, specific weekdays. Three separate salad bar ingredients were found to be likely sources: peeled whole carrots served in a bowl of water, grated carrots, and red peppers. An anthroponotic route of infection was speculated, triggered by a person excreting the parasite which may have had contaminated the buffet [180].

In Norway a searching for parasites in fruits and vegetables was undertaken in the period from 1999 to 2001 [16]. Of the 475 samples, 29 were found to be positive for Cryptosporidium oocysts and Giardia cysts, while 19 only for Cryptosporidium (lettuce and mung bean sprout samples). Mung bean sprouts were significantly more likely to be contaminated with Cryptosporidium oocysts or Giardia cysts than the other fruits and vegetables, despite concentrations were generally low (approximately 3 (oo)cysts per $100 \mathrm{~g}$ product). There was no association between imported produce and detection of parasites. Crvptosporidium oocysts and Giardia cysts were also detected in water samples concerned with field irrigation and production of bean sprouts [16]. This was the first report on detection of parasites in vegetables and fruit obtained in a highly developed wealthy country, without there being an outbreak situation.

4.3. Foodborne Cryptosporidiosis in USA and Canada. Generally, viruses and encysted parasites are more resistant than enteric bacteria to adverse environmental conditions, but all pathogens can survive long enough for transfer from a contaminated worker to food and food contact surfaces 
[176]. Also outbreaks associated with consumption of fruit juice have been growing as an emergent public health problem since the early 1990s, when the first outbreak associated with apple cider was described [181]. However, in the period from September to November 2003, 12 local residents in Northern Ohio were diagnosed with cryptosporidiosis for having drunk ozonated apple cider [182] (Table 4). In response to epidemiologic investigations of outbreaks in which juice is implicated, the USA Food and Drug Administration (FDA) has implemented process control measures to regulate the production of fruit juice, according with the Hazard Analysis Critical Control Point (HACCP) plan. However juice operations that are exempt from processing requirements or do not comply with the regulation continue to be implicated in outbreaks of illness. The CDC receives reports of food-associated outbreaks of illness (Table 3) [183] and its Foodborne Outbreak Reporting System has reviewed, from 1995 through 2005, ten implicating apple juice or cider, eight linked to orange juice, and three involving other types of fruit juice-associated outbreaks. Among the 13 outbreaks of known etiology, two were caused by Cryptosporidium and one by Shiga toxin-producing E. coli O111 and Cryptosporidium [184] (Table 4). The incidence of foodborne disease outbreaks caused by contaminated low$\mathrm{pH}$ fruit juices is highly increasing [185]. The association of Cryptosporidium with fruit juice is raising a safety concern in food industries. In 1998, CDC implemented enhanced surveillance for foodborne-diseases outbreaks (FBDOs) by increasing communication with state, local, and territorial health departments and revising the outbreak report form. Since 2001, reports of FBDOs are submitted through a web internet application called electronic Foodborne Outbreak Reporting System (eFORS) (Table 3) [183, 185-191].

4.4. Foodborne Cryptosporidiosis in South and Central America. In Central America there is a high attention to foodborne infections. Recently, the role of the food handlers has been investigated in Venezuela, where cryptosporidiosis is an important public health problem [192]. Despite a basic investigation approach, fourteen out of 119 fecal samples from food workers were found positive for Cryptosporidium spp. and associated with other protozoa, being most frequent Endolimax nana, followed by B. hominis, Entamoeba coli, G. lamblia, and E. histolytica/Entamoeba dispar. In the paper of Calvo et al. 2004, [193] lettuce, parsley, cilantro, strawberries and blackberries circulating in local agricultural markets of the Central Valley of Costa Rica were investigated for the presence of Cryptosporidium spp., Cyclospora spp., and Microsporidia. Fifty different samples of each product, 25 taken in the dry season and 25 in the rainy season and coming from five different local agricultural markets, were evaluated. Although all vegetables presented fecal coliforms in high concentrations, lettuce and cilantro presented a statistical difference between the rainy and the dry season, being greater during the rainy season. Fecal coliforms were not detected in strawberries and blackberries probably due to its low $\mathrm{pH}$. All products presented Cryptosporidium spp., Cyclospora spp., and Microsporidia. Cryptosporidium was not present in strawberries. Microsporidia were present in all products except blackberries and Cyclospora was only isolated from lettuce during the dry season. These results show the importance of introducing good agricultural practices, especially due to the resistance of Cryptosporidium and Cyclospora to disinfecting agents [193].

4.5. Conclusions. The considerable presence of Cryptosporidium in diversified food matrices makes it imperative to develop appropriate prevention strategies for food safety and suitable molecular techniques for parasite identification. As a general role, the control strategies should be based on the education of the consumers, farmers, and shepherds, the improvement of farming conditions, the improvement or the development of more sensitive methods to detect these parasites in slaughtered animals and in foodstuff, a control of sewage sludge on pastures and of drinking water resources, and the reduction of contacts between livestock and wild animals which frequently represent the most important reservoir of these pathogens [177].

\section{Cryptosporidiosis a "Travelers' Disease"}

5.1. Traveler's Diarrhoea and Main Pathogen Agents. Traveler's diarrhoea (TD) occurs in 20 to $60 \%$ of European or North American travelers in intertropical areas [194]. The main agents are E. coli pathovars followed by enteroinvasive bacteria, enteric viruses, and protozoa ( $G$. intestinalis, C. parvum and E. histolytica). Several studies have shown that a large proportion of travellers and immigrants from tropical and subtropical countries are affected by gastrointestinal disorders and harbour intestinal pathogens without clear gastrointestinal problems [195-200]. Travelling represents an important risk factor for acquiring infection also with sporeforming protozoa as Cyclospora, Microsporidia, and Isospora [201]. Protozoan infections with G. lamblia and C. hominis/C. parvum are the main nonviral causes of diarrhoea in industrialised countries [202] and are even more frequently seen as the cause of gastrointestinal complaints in returning travellers [196, 203, 204].

\subsection{Principal World Regions Associated to Travel-Linked} Transmission. An important study investigated the relationship between Cyclospora infection and seasonality in Turkey [205]. Parasites such as Cryptosporidium, G. intestinalis, E. histolyticaldispar, B. hominis, and others were also observed (Figure 6). The incidence of cyclosporiasis was higher in Summer and early Autumn and most of the Cyclosporainfected patients were without diarrhea. On the other hand, patients with a history of recent travel to a developing country in the tropics usually present persistent diarrhea. However, very mild infections may be underdiagnosed even if causing typical traveler's diarrhea. In a patient with a history of travel and persistent diarrhea unresponsive to the usual antibiotic and antidiarrhea treatment, stool studies for the cited protozoa infections should be always routinely performed (Table 1) (Figure 3). 


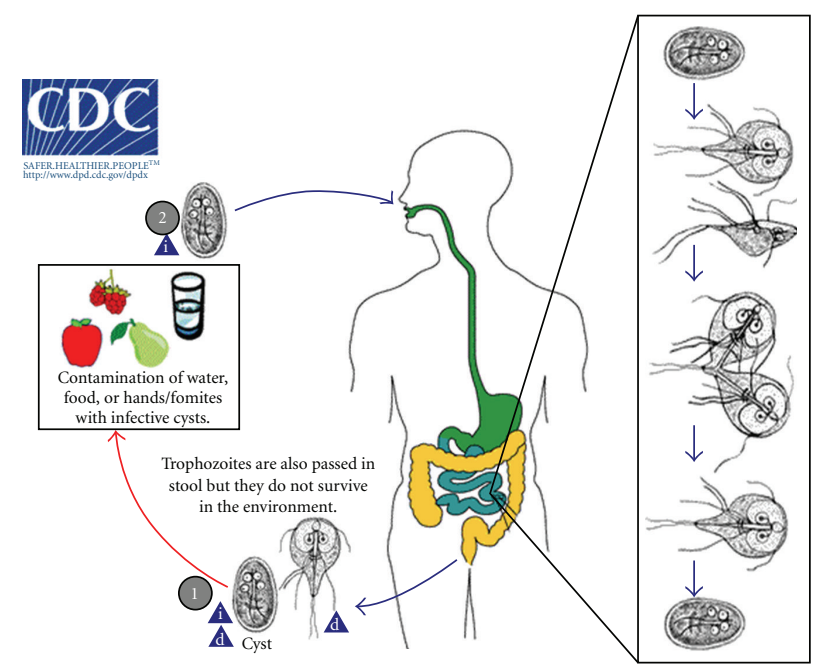

A = Infective stage

A. = Diagnostic stage

(a) Giardia Life Cycle

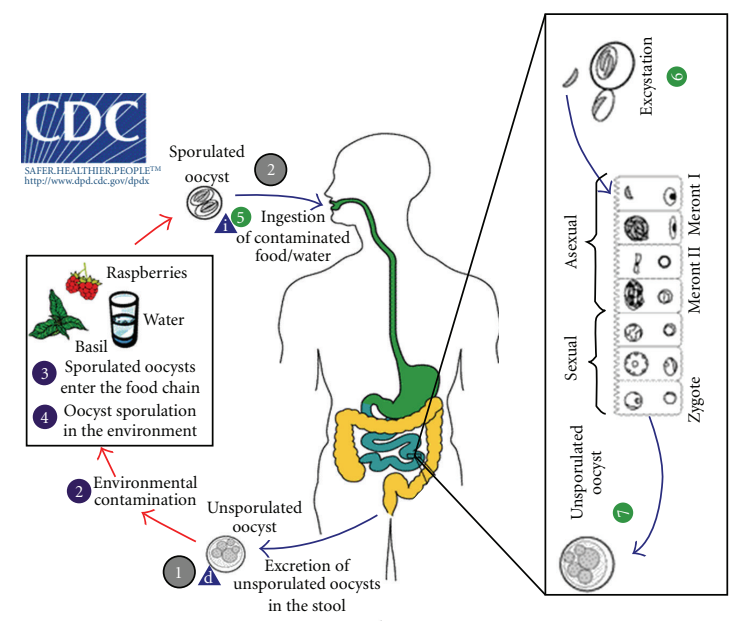

A = Infective stage

A. = Diagnostic stage

(b) Cyclospora Life Cycle

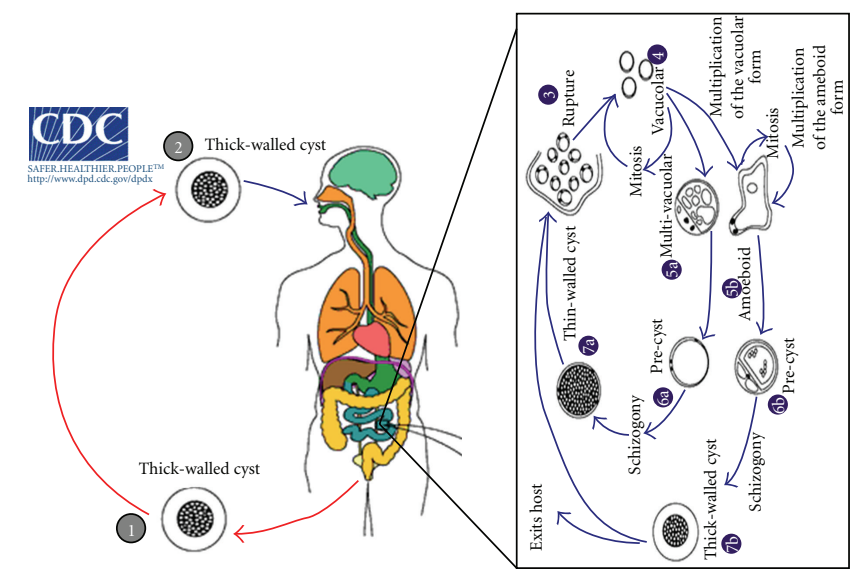

(c) Blastocystis Life Cycle

FIgure 6: Continued. 

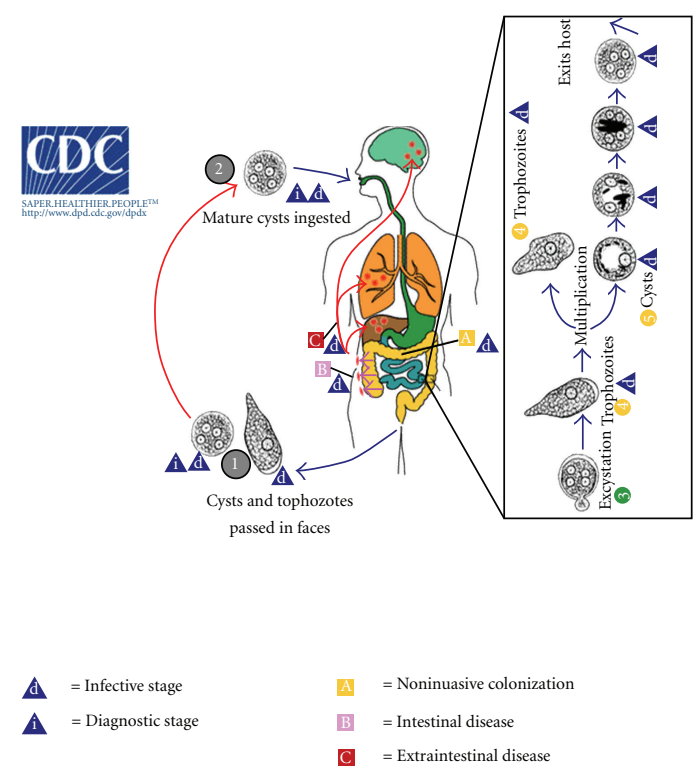

(d) Entamoeba Life Cycle

FIgURE 6: Schematic life cycle of the most recurrent Cryptosporidium coinfective and coemerging parasitic agents. Panel (a). Giardia intestinalis (also called Giardia lamblia or Giardia duodenalis) is a flagellate parasite (Diplomonadida). Both cysts and trophozoites can be found in nondiarrheal feces (diagnostic and infective stages, (1)). Cysts are resistant forms and are responsible for transmission of giardiasis (infective stage, (2)). Infection occurs by the ingestion of water or food contaminated by cysts, or by the fecal-oral route (hands or fomites). In the small intestine, excystation releases trophozoites which multiply by longitudinal binary fission, remaining in the lumen of the proximal small bowel where they can be free or attached to the mucosa. Encystation occurs as the parasites transit toward the colon. Panel (b). Cyclospora cayetanensis is a coccidian parasite (Apicomplexa). When passed in stools, the oocyst is not infective (on the contrary of Cryptosporidium, thus direct fecal-oral transmission cannot occur). In the environment, sporulation occurs after days or weeks, resulting in division of the sporont into two sporocysts, each containing two sporozoites (diagnostic and infective stages, (1)). Fresh food and water can serve as vehicles for transmission and the sporulated oocysts are ingested (infective stage, (2)). The oocysts excyst in the gastrointestinal tract, freeing the sporozoites which invade the epithelial cells of the small intestine. Inside the cells they undergo asexual multiplication and sexual development to mature into oocysts, which will be shed in stools. Panel (c). Blastocystis hominis is a Heterokontid Chromista (Stramenopiles). The thick-walled cyst present in the stools (diagnostic stage, (1)), which varies tremendously in size from 6 to $40 \mu \mathrm{m}$, is believed to be responsible for external transmission, possibly by the fecal-oral route through ingestion of contaminated water or food (infective stage, (2)). The cysts infect epithelial cells of the digestive tract and multiply asexually. Vacuolar forms of the parasite give origin to multi vacuolar and ameboid forms. The multivacuolar form develops into a precyst that gives origin to a thin-walled cyst, thought to be responsible for autoinfection. The ameboid form gives origin to a precyst, which develops into thick-walled cyst by schizogony. The thick-walled cyst is excreted in feces. Panel (d). Entamoeba histolytica/dispar is an Amoebozoa parasite. Cysts and trophozoites are passed in feces (diagnostic and infective stages, (1)), the first found in formed, whereas the latest in diarrheal stool. Infection by E. histolytica occurs by ingestion of mature cysts in fecally contaminated food, water, or hands. Excystation occurs in the small intestine and trophozoites are released, which migrate to the large intestine and multiply by binary fission to produce cysts, where both stages are passed in the feces. Cysts can survive days to weeks in the external environment and are responsible for transmission (diagnostic and infective stages, (2)). Trophozoites passed in the stool are rapidly destroyed once outside the body, and if ingested would not survive exposure to the gastric environment. In many cases, the trophozoites remain confined to the intestinal lumen (noninvasive infection) of individuals who are asymptomatic carriers, passing cysts in their stool. In some patients the trophozoites invade the intestinal mucosa (intestinal disease), or, through the bloodstream, extraintestinal sites such as the liver, brain, and lungs (extraintestinal disease), with resultant pathologic manifestations. It has been established that the invasive and noninvasive forms represent two separate species, respectively, E. histolytica and E. dispar. These two species are morphologically indistinguishable unless E. histolytica is observed with ingested red blood cells (erythrophagocytosis). Infective and diagnostic stages, as well as body organs, are graphically reported, when surely assessed in the life cycle of the parasites. Modified from pictures available at the CDC site for parasite identification and diagnosis (http://www.dpd.cdc.gov/dpdx/HTML/Para_Health.htm). Putignani and Menchella, 2010.

A large study analysed 1,179 North-American travelers who visited Mexico from 2005 to 2007 [206]. TD was reported by 521 participants. A long stay in Mexico was identified as a risk factor for cryptosporidiosis. The Nassau County Health Department (NCHD) in Florida identified an outbreak of gastrointestinal (GI) illness in a returning choral group who toured Ireland in 2006 [207] (Table 4). In the long-term report performed in England and Wales from 2000 to 2003 [63] (Table 2), C. hominis was more prevalent in patients reporting recent foreign travel with late Summer and early Autumn picks [63] (Table 2). However, samples from other UK cases, contracted during foreign travels, were entirely characterised as C. parvum (type 1 and 2) [208] (Table 2). In the paper of Chalmers et al., 2008 [209], 115 
isolates were investigated to assess UK transmission linked to travelling for $C$. hominis. Among the identified subtypes, the predominant was IbA10G2 (Table 2) not apparently linked to recent travel outside Europe [209].

5.3. Conclusions. Person travelling abroad, especially in regions identified as having high risk of infection (e.g., Ireland, UK, Turkey, Mexico) ought to undergo routine testing for intestinal parasites (Figure 4). A large variety of parasitic infections can be expected in homecoming travellers, and diagnostic procedures play a crucial role in the detection of intestinal parasites found in patients with and without gastrointestinal complaints. Although microscopy is considered the gold standard, it is labour intensive and its diagnostic performance critically depends on welltrained microscopists. Enzyme immunoassays [210, 211] and fluorescent antibody assays [212] have been accepted as alternative diagnostic methods for the detection of $G$. lamblia and Cryptosporidium in stools. Currently, the introduction of real-time PCR combining several targets into one multiplex assay offers the possibility of using DNA-based detection techniques in a high-throughput diagnostic approach [213].

\section{Cryptosporidiosis in HIV-Infected Individuals}

6.1. Infection Pathogenesis and Symptoms in HIV Impairment. The prevalence of cryptosporidiosis in HIV-infected patients with diarrhea has been reported to range from 3 to $16 \%$ in developed countries, depending on the population studied, degree of immunosuppression, and use of antiretroviral therapy although it is most frequent in men affected by gay-bowel syndrome $[214,215]$. C. parvum is primarily responsible for watery diarrhoea, but it may also trigger biliary disease, hepatitis, pancreatitis, arthritis, and possibly respiratory tract infections [214, 216]. Diarrhoea is selflimited in immunocompetent individuals or in those whose CD 4 cell counts $>200 / \mathrm{mm}^{3}$, but may be severe, and unremitting or relapsing in severely immunodeficient patients (CD4 cell counts $<100 / \mathrm{mm}^{3}$ ). In these cases chronic infection can lead to dehydration, malnutrition, malabsorption, wasting and, frequently, death [214]. Biliary cryptosporidiosis is more frequent in patients with CD 4 cell counts $<50 / \mathrm{mm}^{3}$ and commonly presents with right upper quadrant pain, nausea, fever, vomiting and often with absence of diarrhea. Coinfection with cytomegalovirus or microsporidia has been frequently found in biliary cryptosporidiosis [216]. All segments of the gastrointestinal tract may be involved, but the small bowel is the main target organ followed by the colon [217]. Esophageal cryptosporidiosis, with parasites attached to the squamous mucosa and the luminal borders of submucosal glands and ducts, has been described both in adults and in children with AIDS [214]. Intestinal coinfection by C. parvum and Cyclospora species or cytomegalovirus is not rarely documented [216, 217] (Figure 6). Recent evidence suggests that epithelial apoptosis mediated by cytotoxic host $\mathrm{T}$ cells might play a role in the development of colonic lesions in AIDS-related cryptosporidiosis [218], suggesting a modified pathogenesis in HIV-positive patients. With the introduction of highly active antiretroviral therapy (HAART), the incidence of cryptosporidiosis has declined and chronic diarrhea and cryptosporidial infection often resolve with increases in CD4 lymphocyte count $[219,220]$. In countries where HAART is available, HIV infection is generally a chronic disease strictly depending on the patient's adherence to treatment [221].

6.2. HIV-Related Cryptosporidiosis in Europe. Recently, in Europe few studies have traced the entire spectrum of epidemiological diffusion routes in HIV-infected patients. In a large study [222], Cryptosporidium isolates from HIV infected and uninfected patients from UK were compared to other isolates collected in different geographical areas (Kenya, Malawi, Brazil and Vietnam). Among the C. parvum group, strains clustered distinctly into either human or bovine genotypes regardless of the geographical origin, age, or HIV status of the patients (Table 2). The intragenotypic variation observed in the $C$. parvum human genotype was wide-ranging compared to that within the C. parvum bovine genotype group. The variation within genotypes was conserved in all geographical regions regardless of the patient HIV status (Table 2). Independent widespreading of genotypes was also observed in the study by Morgan et al., 2000 [223], where isolates from HIV infected patients from Switzerland were compared to other isolates from Kenya and the USA (Table 2). In Portugal, to investigate a possible zoonotic transmission in HIV-seropositive patients, isolates from patients, cattle, sheep and wild ruminants were collected from different regions and appeared largely limited to the only Portugal (Table 2) [78]. A surveillance study on Cryptosporidium in HIV-infected adults was carried out in Spain [224]. C. hominis was detected in $10 \mathrm{HIV-infected} \mathrm{and}$ C. parvum in six HIV-infected individuals showing a similar prevalence of the two species (Table 2).

6.2.1. HIV-Related Cryptosporidiosis in Italy. In Italy, during the previous decade, remarkable epidemiological and clinical studies have been provided [225-229] (Figure 5). An outbreak affected both HIV-positive and HIV-negative members of a drug rehabilitation community in 1995 in Northern Italy (Emilia Romagna) [226]. The 31\% of the HIV-positive individuals were affected, with a severity grade according to CD4 cell count. The Crytosporidium oocysts were identified in the sediment of the water tanks used to store drinking water for the community, suggesting water as the vehicle of infection [226] (Figure 5).

However, following these pivotal studies, only a limited literature on AIDS-related cryptosporidiosis has been produced in the last ten years in Italy, clearly reflecting the positive impact of the HAART therapy on incidence and severity of opportunistic infections.

6.3. HIV-Related Cryptosporidiosis in USA and Canada. An unusual aspect of cryptosporidiosis onset in HIV/AIDS persons was approached by evaluating events of recreational water activities and risk of exposure to Cryptosporidium in 
waterways of Baltimore (Maryland, USA) [230]. Interviews conducted on HIV/AIDS patients showed that approximately $48 \%$ of respondents participated in recreational water activities and had almost equally gender probability to contract waterborne pathogens.

6.4. HIV-Related Cryptosporidiosis in Africa. In Iran ten health centers were mapped for searching of Cryptosporidium in diarrheal patients. The study [231] showed that overall, $1.4 \%$ of all patients and $6.3 \%$ of diarrheal patients were infected by Cryptosporidium while AIDS patients who were suffering from diarrhea reached the $33.4 \%$.

In Equatorial Guinea a study identified C. parvum, C. hominis and C. meleagridis in 35 cases: remarkably over $90 \%$ of the species were isolated from HIV-positive patients (Table 2) [232].

6.5. HIV-Related Cryptosporidiosis in Asia. A prevalence of intestinal parasites in HIV patients in India was determined by testing acute, chronic diarrhoea, and controls without diarrhoea [233]. I. belli was found in $18.6 \%$ of chronic diarrhoea and $7.3 \%$ of acute diarrhoea. Cryptosporidium was detected irrespective of specific clinical signs. Microsporidia and $C$. cayetanensis were detected only in one chronic case. Remarkably, I. belli appeared the predominant parasite associated with diarrhoea among HIV patients, providing an important evidence of a low-represented but emerging parasite in gastrointestinal infections [234]. Reports on the prevalence of cryptosporidial diarrhoea in HIV-infected adults from different parts of India from the mid-1990s have shown a range from 0.7 to $83 \%$ in symptomatic and from 1.4 to $57 \%$ in asymptomatic individuals, with very high rates in both groups and a strong correlation between immune status impairment and diversity of symptoms $[235,236]$. In Taiwan the extremely low prevalence of intestinal cryptosporidiosis among HIV patients [76], despite detection of cryptosporidia in most of the surface waters [168], may be the result of using boiling water $[5,76]$. In Malaysia, the commonness of fecal wastes from human and nonhuman hosts suggests that many environments, particularly water and soil, act as vehicles for the spreading of the disease [237]. A recent paper [238] investigated the occurrence of intestinal parasites in HIV/AIDS patients with chronic diarrhoea in Indonesia. Parasites were found in $84 \%$ of samples (single species infections, $71 \%$; polyparasitism, $13 \%$ ), with protozoan pathogens occurring most commonly. Cryptosporidium, C. cayetanensis, and $G$. duodenalis were the most frequent single infections. Cryptosporidium and C. cayetanensis occurred in 12\% and $8 \%$ of all infections. The most common coinfection was with B. hominis and Cryptosporidium (6.3\%) (Figure 6). No seasonal influence was observed for Cryptosporidium, C. cayetanensis, or B. hominis. A study [239], representing the first genetic identification of Cryptosporidium species in cattle in Thailand, showed that all HIV and cattle stool samples were characterize as C. parvum, suggesting a possible zoonotic transmission for HIV individuals (Table 2) [239].
6.6. HIV-Related Cryptosporidiosis in South and Central America. In Perù, a study on the genetic diversity of Cryptosporidium spp. in HIV-positive people [240] suggested that $C$. hominis is the predominant species in HIV patients, while zoonotic Cryptosporidium spp. accounts for about $30 \%$ of cases (Table 2) [240]. A prospective longitudinal cohort study [241] conducted in Haiti showed that AIDS patients were infected by either human or animal genotypes. These data confirm that immunocompromised individuals are susceptible to a wide range of Cryptosporidium spp. which remains a frequent hazard especially in countries with poor hygiene and overcrowded conditions associated with urban slums [69].

6.7. Conclusions. In developing countries with no or limited access to HAART, AIDS is rapidly expanding with a high fatality ratio (Figure 4). Furthermore, new HAART baselines, where introduced, are now modifying HIV circulation modes and opportunistic infections in these geographic areas. The data on the parasite's transmission in the SubSaharan Africa clearly show high rates of severe or even fatal Cryptosporidium infections, massively contributing to the entire worldwide burden of sporadic cases (Figure 4). In HIV patients the impact of $C$. felis infection in tropical countries is becoming an emerging issue. In developed countries (Figure 4), therapeutic approaches are effective in reducing fecal output, but the eradication of the parasite is rarely obtained. Cryptosporidiosis is still a leading opportunistic infection in HIV persons and, despite HAART therapy, it should not be underestimated in epidemiological tracing and clinical followup of these patients. Immunocompromised persons should be cautioned on the potential risks from recreational water contact and their water-related practices should always be considered in the clinical monitoring of their health status.

\section{Cryptosporidiosis in Children}

7.1. General Notes. In the early 1980s, diarrhoeal disorders were the biggest child killers, responsible for an estimated 4.6 million deaths worldwide every year. Despite widespread use of oral rehydration therapies and an increased understanding of the pathogenesis of diarrhoea, 2.5 million children still die from these illnesses every year, almost all of them in developing countries [242]. Parasites such as Cryptosporidium and Giardia are leading agents of chronic or persistent diarrhoea worsened by specific risk factors such as malnutrition or immune deficiency [243].

7.2. Children Cryptosporidiosis in Europe. Studies on cryptosporidiosis in children have been progressively developed in the last few years in Europe [35, 244]. Survey laboratory practices in the UK have recently included screening of all fecal specimens from children aged 15 or younger, with routine reports to the Communicable Disease Surveillance Centre (CDSC) of the PHLS (Public Health Laboratory Service) (Table 3) [245]. Among the described outbreaks (see Sections 3.1 and 3.2), in many episodes the children represented the largest portion of confirmed cases of cryptosporidiosis 
[84, 246-251] (Table 4). The work by Chalmers et al., 2009 [63] showed that the epidemiology of human cryptosporidiosis in UK, from 2000 to 2003, importantly differed among Cryptosporidium infecting species with reference to children age groups (Table 1) (Figure 3 ). The mean age of $C$. parvum cases was lower than that of $C$. hominis cases. However, an opposite trend in infants under one year, independently from the gender and possibly linked to the stay of the babies in day-care nurseries, was observed. A seasonal distribution of cryptosporidiosis in children in a region of North-Eastern Spain, was determined [53] (see Introduction, Section 1.3.2). Prevalence was highest in children aged 1 to 3 years old and significantly more elevated in the Autumn-Winter period than in the Spring-Summer period [53]. Furthermore, the stay within a nursery and the improper diaper usage, for a group of 24 day-care children, were analysed as triggering factors in the community outbreak described by Ortega et al. in 2006 in Spain [252] (Table 4). In Spring 1998, an acute gastroenteritis outbreak, which mainly affected preschool children, took place in Guadarrama (Spain) (Table 4) [253]. In Spain a large surveillance study on a set of stool samples collected from Cryptosporidium-infected patients, including 92 children [224], revealed a high heterogenicity of species (Table 2). A recent study [254] performed, amongst the others, on 32 hospitalised children, revealed an impairmentdependant prevalence of Cryptosporidium species in hospitalised children [254] (Table 2). Large-scale surveys of representative population groups in the Central and NorthWestern regions of the Russian Federation showed a mean population incidence of $3.3 \%$, much higher in children $(3.7 \%)$ than in adults $(0.4 \%)$. There were differences in the infection rates between genders (boys more affected than girls) but not between rural and urban children [255].

7.2.1. Children Cryptosporidiosis in Italy. A large group of 618 children with diarrhea was prospectively evaluated for viral, bacterial, and parasitic enteric pathogens in a multicenter study performed by the Italian Study Group on Gastrointestinal Infections [256] (Figure 5). The agents mainly associated with disease were Rotavirus, Salmonella, and Campylobacter. Cryptosporidium and Giardia were observed only in 10 patients [256].

Another important study [257] evaluated the prevalence of C. parvum in 368 hospitalized children with enteritis, of whom 359 were immunocompetent and 9 were HIVinfected. C. parvum oocysts were found in seven out of 368 specimens. All subjects with cryptosporidiosis were living in Apulia (South Italy) and had not travelled outside of Italy. No differences between those living in urban or rural areas was observed and no correlation was found between seasonal timing of specimen collection and positivity for C. parvum. Importantly, the areas were served by chlorinated water systems. In two out of seven children the parasite was associated also with $S$. typhimurium in one case and Rotavirus spp. in the other. The population study on the drug community members [226], included the subset of their 135 children. Interestingly, 28 out of 135 children, aged in the range 0-12 years, were affected by Cryptosporidium. Updated data on cryptosporidosis prevalence in pediatric population are nowadays missing, despite the growing clinical interest on children cryptosporidiosis.

7.3. Children Cryptosporidiosis in USA and Canada. In 2007 the Idaho waterborne outbreak in a municipal park [258] (Table 4) affected, over 50 ill people, 36 children with a peak ranging in the 4-6 age group. In 2006, in a waterborne outbreak the $83 \%$ of primary cases occurred in children [85] (Table 4). Also in the waterborne outbreak registered in Illinois in 2001, children were predominantly involved [259]. Interestingly, C. hominis was the only etiological agent and one of the risk agents was the heavy usage of recreational water by diaper-aged children (Figure 3) (Table 4). Also in the outbreak registered in Florida in 1999 [260] (Table 4), over the 86 park visitors interviewed, the 38 which had gastroenteritis were 8 year old over an age range of 2-65 years. During an outbreak of giardiasis and cryptosporidiosis in Central Florida in September 2006, including also coinfection (Figure 6), only children were affected [261] (Table 4).

Currently, the number of internationally adopted children worldwide has rapidly increased during the past decade, providing an additional surveillance indicator for cryptosporidiosis acquired in their country of origin. The work of Saiman [195] performed a retrospective cohort study on 504 children adopted from abroad in the USA from 1997 to 1998 to determine the prevalence of infectious diseases. Being born in Eastern Europe was a risk factor for the acquisition of G. lamblia. Thirty-two children had one or more organisms identified by stool microscopy: B. hominis, Dientamoeba fragilis, E. nana, Hymenolepis nana, Ascaris lumbricoides, Chilomastix mesnili, and Entamoeba hartmanni were detected. Cryptosporidium species were identified only in four out of 504 children, but probably underestimated for the low sensitivity of the direct fluorescent method.

7.4. Children Cryptosporidiosis in New Zealand and Australia. An outbreak affecting eight children in Australia was associated to a contamination of drinking unpasteurised milk [262] (Table 4). Despite being rarely observed, a pivotal work [263] described a previous children outbreak of cryptosporidiosis linked to drinking school milk in September 1995 in the UK. The only exposure significantly associated with illness was drinking school milk, possibly infected by a temporarily failing pasteurisation plant at the local producing farm. A children community outbreak was also reported in New Zealand in 2001 [264] (Table 4). The 19 cases aged under 7 years, were linked to a specific farm event identified as parasite hand-to-mouth transfer after touching an infected calf.

7.5. Children Cryptosporidiosis in Africa. C. parvum is a leading pathogen in children in African developing countries. Here, as in other low-income areas, with no or limited access to HAART, AIDS is rapidly expanding in infants [265]. The fatality rate increased due to opportunistic infections, with $C$. parvum being one of the leading agents 
of severe diarrhea in infants affected by HIV/AIDS [265]. However, stunting is a major burden in developing countries, affecting $\sim 147$ million children. Repeated or prolonged episodes of diarrhoea during childhood increase the risk of stunting, which is believed to be associated with significant morbidity. Although the relationships between malnutrition, environmental and diarrhoeal illnesses are complex, studies have suggested a connection between stunting and diarrhoea causing pathogens, including G. lamblia, C. parvum, and enteroaggregative E. coli (EAggEC) [266]. A recent paper [267], showed that the microsporidian parasite E. bieneusi is associated with lower rates of weight gain in children with persistent diarrhea in Uganda. Children with microsporidiosis were predicted to weigh $1.3 \mathrm{~kg}$ less than children without microsporidiosis at 5 years of age [267]. The benefits of exclusive breastfeeding for health in infants have been widely described. The study by Bilenko et al., 2008 [126], considered whether partial breastfeeding has protective effects against enteric infection and associated morbidity in population where early addition of supplementation is common. In this study, 238 Bedouin infants were followed from birth to 18 months. Exclusive breastfeeding was protective against infection and morbidity at ages 0 to 3 months. In the age range of 4 to 6 months, partial versus nonbreastfeeding was associated with lower rates of infection with Cryptosporidium spp. and Campylobacter spp. In older children (10-12 month age range) partial breastfeeding as compared to none, protected against infections with Cryptosporidium spp. and G. lamblia. Short-term protection from maternal antibodies passed to infants during breastfeeding may result in a lack of cryptosporidial infection in infancy. This protection of breastfeeding children may, however, result in such children developing less anti-Cryptosporidium immunity of their own, so that, by school age, the children who had been breastfed are those most likely to be found infected [268]. Hospital- and community-based studies in Sub-Saharan Africa document a high prevalence of cryptosporidiosis in children aged 6-36 months, particularly among those who are malnourished or HIV-positive and during rainy seasons. Transmission appears to occur predominantly through an anthroponotic cycle [269]. Prevalence of Cryptosporidium and Giardia infections was assessed among children using protected and unprotected water sources in Eastern Ethiopia, in November 2005 to May 2006 [270]. Of 655 children examined, 80 were infected with Cryptosporidium and 231 with Giardia. No difference was observed in the prevalence of cryptosporidiosis and giardiasis between children drinking water from protected and unprotected sources [270]. The study of Dlamini et al., 2005 [271], reports the first finding on Cryptosporidium spp. detection among children of the Swazi ethnic group in Zimbabwe. A study focused on the prevalence of cryptosporidiosis in pediatric hospital patients in Niger [272], where malnutrition and diarrhoea are two major public health issues. The aim of this study was to get a first evaluation on the prevalence of Cryptosporidium spp. in the stools of hospitalized children younger than 5 years of age. The weight/age ratio to describe malnutrition was calculated and analyzed with the Epi-Info software $[273,274]$, (Table 3 ). In the three months study
220 children were included (mean age 20 months) showing that $65 \%$ of the children were suffering from moderate and severe malnutrition. Diarrhoea was reported in 52\% of the children. Cryptosporidium oocysts were detected in 12 out of 220 children with 10 children malnourished. A study on children (median age 13.5 months) presenting with acute diarrhea and rehydration clinics in Madagascar, was undertaken between May 2004-May 2005 [275]. Twelve cases of cryptosporidiosis were detected only in the rainy season. As 11 of the 12 cases were caused by C. hominis and only one by $C$. parvum, most of the cases were probably the result of anthroponotic transmission (Table 4) [275]. A large study was performed in Kuwait to investigate the incidence of cryptosporidial infection in children presenting with gastrointestinal symptoms at the local hospitals [54]. Over a period of three years, fecal samples from 3549 children were analyzed for the presence of Cryptosporidium oocysts, detected in 51 children with diarrhea. Prevalence was highest in children older than two years of age. The maximum number of cases was seen during the months January to April, indicating a marked seasonal variation. Three possible modes of infection transmission were inferred: (i) drinking contaminated water stored in overhead water tanks; (ii) person to person; (iii) contact with infected animals. A common polyparasitism was mainly due to the recurrent Cryptosporidium co-infective Giardia and Cyclospora parasites, as also reported by recent outbreak reports [261, 276, 277] (Table 4), confirming these parasites as largely emerging pathogens $[1,201]$ also with $B$. hominis and $E$. histolyticaldispar [278] (Figure 6).

7.6. Children Cryptosporidiosis in Asia. An interesting correlation between subtypes distribution and geographical settings for children infections, was investigated to identify geographical-dependent variation [36] (Table 2). In this study, species, genotypes, and subgenotypes of Cryptosporidium spp. infections were identified for the first time in a well-defined cohort of children in Southern India. Only one previous report was produced on genotypes distribution of Cryptosporidium spp. in children in Eastern India [57]. C. parvum-positive samples revealed that all were subgenotype Ic, usually associated with anthroponotic transmission (Table 2). There were no significant differences in demographic or clinical (nutritional status, vomiting, fever) characteristics between C. hominis and C. parvum or C. felis [87] infected children and those infected with different subgenotypes. However, C. hominis-infected children had a significantly greater severity of diarrhea [36]. There was also a trend toward a longer average duration of diarrhea in C. hominis-infected children than in those infected with other species (Table 1) (Figure 3). There were two significant time clusters of cryptosporidial diarrhea, one during February-March and the other during June-August. In the other study of Ajjampur [235], fecal samples from 158 children with and 99 children without diarrhoea were tested for enteric pathogens in Southern India. Remarkably, Cryptosporidium spp. resulted in one of the most common causes of diarrhoea in hospitalized children [235, 236]. 
The epidemiology, clinical features, nutritional status, and causative agents of diarrhea were studied in 289 children in Bangladesh [279]. Compared with malnourished and/or stunted children, better-nourished children experienced significantly fewer diarrheal episodes. G. lamblia, C. parvum, and $E$. histolytica were the most common protozoan agents. A very recent study [280] performed in Bangladesh, examined whether malnutrition, may increase the risk of diarrhea equally for all enteropathogens in infants. Two hundred eighty-nine Bangladeshi children, 2 to 5 years of age, were included in the study. Malnutrition was present in 39\% of the children. Of the identified enteropathogens from stool samples, only enterotoxigenic E. coli, Cryptosporidium spp., and E. histolytica were significantly more prevalent in malnourished children, suggesting that malnutrition may represent a differential risk for enteric pathogens associated with diarrheal illness. A study [281] was conducted to investigate the presence of intestinal parasites among 475 preschool children (aged 3 months to 5 years) in Thailand. The most frequent parasites identified were G. lamblia and Cryptosporidium spp. Highest proportion of intestinal parasites occurred during the rainy season (June-October). A 5-year hospital-based retrospective analysis was aimed to find out the intestinal protozoal parasitic profile in 1790 preschool and school-age children visiting the hospital with gastrointestinal illness in Nepal [282]. G. lamblia was the most prevalent pathogenic protozoan intestinal parasite, followed by E. histolytica. Interestingly, opportunistic pathogens like C. cayetanensis and Cryptosporidium spp. were detected in immunocompromised children below two years of age as a result of vertical transmission, which is alarming for a country like Nepal presenting a "concentrated epidemic" HIV infection period [282]. Intestinal parasites are still a major health problem in Turkey. The study of Börekçı and Uzel [283] identified one or more parasites in $43 \%$ of the children. G. intestinalis was found to be the most common parasite, followed by E. histolytica plus E. coli, E. coli, E. nana, and Cryptosporidium spp. In Turkey, the first waterborne outbreak of cryptosporidiosis with Cyclospora coinfection mainly affected children aged between 0 and 14 years [276] (Table 4) (Figure 6). An interesting topic for children infections in an Asian high-income country, was discussed by Matsubayashi et al., 2005, on Cryptosporidium and Giardia transmission in a zoo in Japan [284]. Cryptosporidium spp. was found only in a raccoon dog, and Giardia spp. was detected in a mandarin duck and two ruddy shelducks. These results corroborate the idea that infected animals could serve as a direct source of contamination for children. In a recent compendium [285], recommendations for public health officials, veterinarians, animal venue staff members and exhibitors, visitors to animal venues, and physicians have been provided. Pet and wild animal transmission has been more critically revised as an important reservoir for cryptosporidiosis in children $[78,118,284,286]$.

7.7. Children Cryptosporidiosis in South and Central America. In South America, cryptosporidiosis is often observed as a pediatric disease in areas where Cryptosporidium spp. are endemic (e.g., Perù). Children < two years of age are frequently infected in community and hospital settings. The spectrum of symptoms is diverse, ranging from acute, severe chronic diarrhea, or vomiting to asymptomatic infections. In a community-based study in Perù, about $30 \%$ of immunocompetent children with cryptosporidiosis reported diarrhea [50]. This four-year study was fundamental for size of sampling set (533 children enrolled), age of children (median age corresponding to 14 days), number of stool specimens $(44,042)$, and Cryptosporidium species identified (Table 2). Children infected with $C$. hominis had higher parasite excretion scores [287] than those infected with other species of Cryptosporidium. Associated clinical manifestations at first infection varied among different Cryptosporidium spp. and genotypes (Table 2). In general, distribution of species was similar to that found in a previous study on Peruvian HIV population [288], where all C. parvum specimens were described to belong to subtype family IIc [87]. In Haiti, the study by Raccurt et al. 2006 [69], highlighted the water contamination in urban slum conditions as a major risk of cryptosporidiosis in children. The considered risk factors, including age at first infection, hygiene parameters, presence of animals, house infrastructure, and indirect economic indicators did not provide statistically significant correlation to Cryptosporidium spp. or subtype families and, therefore, were not identified as risk factors, apparently in contrast to transmission of Cryptosporidium spp. in industrialized nations. A seroepidemiologic study [289] was conducted in Guatemala to determine the feasibility of using antibody markers as indicators of waterborne pathogen infection in rural areas of this country. The prevalence of antibodies was lowest in children 6-12 months old compared with the older age groups (up to 36 months) but still detectable, providing a useful screening tool for the main waterborne pathogens [289]. Such an approach may provide a suitable tool for determining the prevalence of infection in very young children. Molecular characterisation of Cryptosporidium infections among Cuban children (aged 2-8 years) with diarrhoea, were described by Pelayo et al., 2008 [268]. In this study, in contrast with a previous report on intestinal coccidia in Cuban pediatric patients [290], differences in symptoms were detected, possibly age-related.

7.8. Conclusions. Infectious diarrheal diseases remain an important cause of childhood morbidity in developed countries. The recent confirmation of an infection route of Cryptosporidium linked to drinking unpasteurised milk highlights the need to evaluate the entire transmission variety of the parasite, besides the already well-known and identified classical courses of disease in children. In pediatric populations, prevalence data are still underestimated, due to a poor clinical valuation of pathognomic symptoms and to the absence of advanced laboratory tools in diagnostic routine panels. Literature regarding developing countries shows that anthroponotic transmission is the principal mode of infection in children population, while less representative are the water or environmental sources contaminated with zoonotic Cryptosporidium genotypes, a direct 
contact with animals or the presence within farms and/or associated waters. Interestingly, of the 71 Cryptosporidiumlinked outbreaks (Table 4), 17 outbreaks (23.9\%), defined as community-linked, appear to involve predominantly children, underlying the person-to-person contact as the prevailing transmission route. Geographically, this type of outbreaks seem to be concentrated in the USA and in the UK but have been also mapped in New Zealand and South-America (Table 3) (Figure 4). The high heterogenicity of $C$. hominis genotypes, notified in many developing areas for childhood, represents an indicator of endemicity for the transmission of cryptosporidiosis (Figure 4). In the poorest areas, gastrointestinal parasitosis, enhanced by malnutrition, play a major role in children with severe immune impairment, with $C$. hominis being the leading agent of severe diarrhea. Intestinal dysfunction contributes to growth failure and further immune derangement, leading to wasting, and significantly enhancing children mortality. New areas of research on the relationship between breastfeeding and onset/progression of the cryptosporidiosis should be explored, especially in children population characterised by paucity of hygiene, nutrition and hydration, particularly in the first months of life. Lastly, the growing number of internationally adopted children requires an appropriate surveillance to ensure the long-term health of adopted children as well as their families. Thus, periodic surveys of large cohorts of internationally adopted children are now essential to monitor global changing epidemiologic trends.

\section{Acknowledgments}

This paper was supported by a Grant from Ricerca Corrente, Bambino Gesù Hospital and Research Institute (OPBG): "The early Apicomplexa protists as a new model for the understanding of human infectious diseases and pathologies: a novel approach to treat emerging public health challenges through global technological platforms as DNA, protein expression profiling and phylogenomics" (RC 200702P002153). The authors wish to thank Professor Gian Franco Bottazzo, who has enthusiastically given them the idea to put in writing a review on the current picture of global cryptosporidiosis. The authors like to thank Manuela Onori, Federica Del Chierico for paper editing and Eva Ruth Palmieri for English revision. The authors also acknowledge the contribution of the anonymous reviewers whose constructive criticism was of guidance in revision of this paper.

\section{References}

[1] R. W. Goodgame, "Understanding intestinal spore-forming protozoa: cryptosporidia, microsporidia, Isospora, and Cyclospora," Annals of Internal Medicine, vol. 124, no. 4, pp. 429-441, 1996.

[2] L. Eckmann, "Small bowel infections," Current Opinion in Gastroenterology, vol. 18, no. 2, pp. 197-202, 2002.

[3] K. K. Pierce and B. D. Kirkpatrick, "Update on human infections caused by intestinal protozoa," Current Opinion in Gastroenterology, vol. 25, no. 1, pp. 12-17, 2009.
[4] World Health Organisation, "WHO/PAHO informal consultation on intestinal protozoal infections," Tech. Rep. WHO/CDS/IPI/92.2, World Health Organisation, Geneva, Switzerland, 1992.

[5] X.-M. Chen, J. S. Keithly, C. V. Paya, and N. F. LaRusso, "Cryptosporidiosis," The New England Journal of Medicine, vol. 346, no. 22, pp. 1723-1731, 2002.

[6] P. Karanis, C. Kourenti, and H. Smith, "Waterborne transmission of protozoan parasites: a worldwide review of outbreaks and lessons learnt," Journal of Water and Health, vol. 5, no. 1, pp. 1-38, 2007.

[7] H. V. Smith, S. M. Cacciò, N. Cook, R. A. B. Nichols, and A. Tait, "Cryptosporidium and Giardia as foodborne zoonoses," Veterinary Parasitology, vol. 149, no. 1-2, pp. 29-40, 2007.

[8] S. M. Cacciò and E. Pozio, "Molecular identification of food-borne and water-borne protozoa," The Southeast Asian Journal of Tropical Medicine and Public Health, vol. 32, no. 2, pp. 156-158, 2001.

[9] S. M. Cacciò, "Molecular epidemiology of human cryptosporidiosis," Parassitologia, vol. 47, no. 2, pp. 185-192, 2005.

[10] A. Grinberg, N. Lopez-Villalobos, W. Pomroy, G. Widmer, H. Smith, and A. Tait, "Host-shaped segregation of the Cryptosporidium parvum multilocus genotype repertoire," Epidemiology and Infection, vol. 136, no. 2, pp. 273-278, 2008.

[11] H. L. DuPont, C. L. Chappell, C. R. Sterling, P. C. Okhuysen, J. B. Rose, and W. Jakubowski, "The infectivity of Cryptosporidium parvum in healthy volunteers," The New England Journal of Medicine, vol. 332, no. 13, pp. 855-859, 1995.

[12] P. C. Okhuysen, C. L. Chappell, J. H. Crabb, C. R. Sterling, and H. L. DuPont, "Virulence of three distinct Cryptosporidium parvum isolates for healthy adults," The Journal of Infectious Diseases, vol. 180, no. 4, pp. 1275-1281, 1999.

[13] L. Jokipii and A. M. M. Jokipii, "Timing of symptoms and oocyst excretion in human cryptosporidiosis," The New England Journal of Medicine, vol. 315, no. 26, pp. 1643-1647, 1986.

[14] C. L. Chappell, P. C. Okhuysen, C. R. Sterling, and H. L. DuPont, "Cryptosporidhim parvun: intensity of infection and oocyst excretion patterns in healthy volunteers," The Journal of Infectious Diseases, vol. 173, no. 1, pp. 232-236, 1996.

[15] B.-M. Hsu, C. Huang, and J. R. Pan, "Filtration behaviors of Giardia and Cryptosporidium-ionic strength and pH effects," Water Research, vol. 35, no. 16, pp. 3777-3782, 2001.

[16] L. J. Robertson and B. Gjerde, "Occurrence of parasites on fruits and vegetables in Norway," Journal of Food Protection, vol. 64, no. 11, pp. 1793-1798, 2001.

[17] R. Reinoso, E. Becares, and H. V. Smith, "Effect of various environmental factors on the viability of Cryptosporidium parvum oocysts," Journal of Applied Microbiology, vol. 104, no. 4, pp. 980-986, 2008.

[18] J. M. Shields, V. R. Hill, M. J. Arrowood, and M. J. Beach, "Inactivation of Cryptosporidium parvum under chlorinated recreational water conditions," Journal of Water and Health, vol. 6, no. 4, pp. 513-520, 2008.

[19] J. Šlapeta, "Centenary of the genus Cryptosporidium: from morphological to molecular species identification," in Giardia and Cryptosporidium: From Molecules to Diseases, G. Ortega-Pierres, S. M. Cacciò, R. Fayer, T. G. Mank, H. V. Smith, and R. C. A. Thompson, Eds., chaper IV, pp. 31-50, CABI, Oxfordshire, UK. 
[20] U. M. Ryan and L. Xiao, "Molecular epidemiology and typing of non-human isolates of Cryptosporidium," in Giardia and Cryptosporidium: From Molecules to Diseases, G. OrtegaPierres, S. M. Cacciò, R. Fayer, T. G. Mank, H. V. Smith, and R. C. A. Thompson, Eds., chaper VI, pp. 65-80, CABI, Oxfordshire, UK.

[21] U. M. Morgan, L. Xiao, R. Fayer, A. A. Lal, and R. C. A. Thompson, "Variation in Cryptosporidium: towards a taxonomic revision of the genus," International Journal for Parasitology, vol. 29, no. 11, pp. 1733-1751, 1999.

[22] C. P. Raccurt, "Worldwide human zoonotic cryptosporidiosis caused by Cryptosporidium felis," Parasite, vol. 14, no. 1, pp. 15-20, 2007.

[23] F. Leoni, C. I. Gallimore, J. Green, and J. McLauchlin, "Characterisation of small double stranded RNA molecule in Cryptosporidium hominis, Cryptosporidium felis and Cryptosporidium meleagridis," Parasitology International, vol. 55, no. 4, pp. 299-306, 2006.

[24] B. Wolska-Kusnierz, A. Bajer, S. M. Cacciò et al., "Cryptosporidium infection in patients with primary immunodeficiencies," Journal of Pediatric Gastroenterology and Nutrition, vol. 45, no. 4, pp. 458-464, 2007.

[25] L. Xiao, V. A. Cama, L. Cabrera, Y. Ortega, J. Pearson, and R. H. Gilman, "Possible transmission of Cryptosporidium canis among children and a dog in a household," Journal of Clinical Microbiology, vol. 45, no. 6, pp. 2014-2016, 2007.

[26] L. Xiao, C. Bern, J. Limor et al., "Identification of 5 types of Cryptosporidium parasites in children in Lima, Peru," The Journal of Infectious Diseases, vol. 183, no. 3, pp. 492-497, 2001.

[27] O. Ditrich, L. Palkovic, J. Stěrba, J. Prokopic, J. Loudová, and M. Giboda, "The first finding of Cryptosporidium baileyi in man,” Parasitology Research, vol. 77, no. 1, pp. 44-47, 1991.

[28] I. Abubakar, S. H. Aliyu, C. Arumugam, P. R. Hunter, and N. K. Usman, "Prevention and treatment of cryptosporidiosis in immunocompromised patients," Cochrane Database of Systematic Reviews, no. 1, Article ID CD004932, 2007.

[29] L. M. Kortbeek, "Clinical representaion in Cryptosporidiuminfected patients," in Giardia and Cryptosporidium: From Molecules to Diseases, G. Ortega-Pierres, S. M. Cacciò, R. Fayer, T. G. Mank, H. V. Smith, and R. C. A. Thompson, Eds., chaper XI, pp. 131-137, CABI, Oxfordshire, UK.

[30] D. A. Mosier and R. D. Oberst, "Cryptosporidiosis: a global challenge," Annals of the New York Academy of Sciences, vol. 916, pp. 102-111, 2000.

[31] J. M. Balbus and M. A. Embrey, "Risk factors for waterborne enteric infections," Current Opinion in Gastroenterology, vol. 18, no. 1, pp. 46-50, 2002.

[32] A. L. Reingold, "Outbreak investigations-a perspective," Emerging Infectious Diseases, vol. 4, no. 1, pp. 21-27, 1998.

[33] S. Kato, L. Ascolillo, J. Egas et al., "Waterborne Cryptosporidium oocyst identification and genotyping: use of GIS for ecosystem studies in Kenya and Ecuador," The Journal of Eukaryotic Microbiology, vol. 50, pp. 548-549, 2003.

[34] S. Hughes, Q. Syed, S. Woodhouse et al., "Using a geographical information system to investigate the relationship between reported cryptosporidiosis and water supply," International Journal of Health Geographics, vol. 3, article 15, 2004.

[35] I. R. Lake, F. C. D. Harrison, R. M. Chalmers et al., "Casecontrol study of environmental and social factors influencing cryptosporidiosis," European Journal of Epidemiology, vol. 22, no. 11, pp. 805-811, 2007.
[36] S. S. R. Ajjampur, B. P. Gladstone, D. Selvapandian, J. P. Muliyil, H. Ward, and G. Kang, "Molecular and spatial epidemiology of cryptosporidiosis in children in a semiurban community in South India," Journal of Clinical Microbiology, vol. 45, no. 3, pp. 915-920, 2007.

[37] A. R. Jex and R. B. Gasser, "Analysis of the genetic diversity within Cryptosporidium hominis and Cryptosporidium parvum from imported and autochtonous cases of human cryptosporidiosis by mutation scanning," Electrophoresis, vol. 29, no. 20, pp. 4119-4129, 2008.

[38] A. R. Jex, A. Pangasa, B. E. Campbell et al., "Classification of Cryptosporidium species from patients with sporadic cryptosporidiosis by use of sequence-based multilocus analysis following mutation scanning," Journal of Clinical Microbiology, vol. 46, no. 7, pp. 2252-2262, 2008.

[39] H.-P. Beck, D. Blake, M.-L. Dardé et al., "Molecular approaches to diversity of populations of apicomplexan parasites," International Journal for Parasitology, vol. 39, no. 2, pp. 175-189, 2009.

[40] A. Pangasa, A. R. Jex, B. E. Campbell et al., "High resolution melting-curve (HRM) analysis for the diagnosis of cryptosporidiosis in humans," Molecular and Cellular Probes, vol. 23, no. 1, pp. 10-15, 2009.

[41] Y. Feng, N. Li, L. Duan, and L. Xiao, "Cryptosporidium genotype and subtype distribution in raw wastewater in Shanghai, China: evidence for possible unique Cryptosporidium hominis transmission," Journal of Clinical Microbiology, vol. 47, no. 1, pp. 153-157, 2009.

[42] M. E. Mallon, A. MacLeod, J. M. Wastling, H. Smith, and A. Tait, "Multilocus genotyping of Cryptosporidium parvum type 2: population genetics and sub-structuring," Infection, Genetics and Evolution, vol. 3, no. 3, pp. 207-218, 2003.

[43] M. Mallon, A. MacLeod, J. Wastling, H. Smith, B. Reilly, and A. Tait, "Population structures and the role of genetic exchange in the zoonotic pathogen Cryptosporidium parvum," Journal of Molecular Evolution, vol. 56, no. 4, pp. 407-417, 2003.

[44] G. Widmer, X. Feng, and S. Tanriverdi, "Genotyping of Cryptosporidium parvum with microsatellite markers," Methods in Molecular Biology, vol. 268, pp. 177-187, 2004.

[45] S. M. Cacciò, "New methods for the diagnosis of Cryptosporidium and Giardia," Parassitologia, vol. 46, no. 1-2, pp. 151-155, 2004.

[46] S. Tanriverdi and G. Widmer, "Differential evolution of repetitive sequences in Cryptosporidium parvum and cryptosporidium hominis," Infection, Genetics and Evolution, vol. 6, no. 2, pp. 113-122, 2006.

[47] L. Xiao, "Molecular epidemiology of cryptosporidiosis: an update," Experimental Parasitology, vol. 124, no. 1, pp. 80-89, 2010.

[48] L. Xiao and U. M. Ryan, "Cryptosporidiosis: an update in molecular epidemiology," Current Opinion in Infectious Diseases, vol. 17, no. 5, pp. 483-490, 2004.

[49] S. M. Cacciò, R. C. A. Thompson, J. McLauchlin, and H. V. Smith, "Unravelling Cryptosporidium and Giardia epidemiology," Trends in Parasitology, vol. 21, no. 9, pp. 430437, 2005.

[50] V. A. Cama, C. Bern, J. Roberts et al., "Cryptosporidium species and subtypes and clinical manifestations in children, Peru," Emerging Infectious Diseases, vol. 14, no. 10, pp. 1567$1574,2008$. 
[51] A. Zintl, A. F. Proctor, C. Read et al., "The prevalence of Cryptosporidium species and subtypes in human faecal samples in Ireland," Epidemiology and Infection, vol. 137, no. 2, pp. 270-277, 2009.

[52] G. Wilkes, T. Edge, V. Gannon et al., "Seasonal relationships among indicator bacteria, pathogenic bacteria, Cryptosporidium oocysts, Giardia cysts, and hydrological indices for surface waters within an agricultural landscape," Water Research, vol. 43, no. 8, pp. 2209-2223, 2009.

[53] A. Clavel, J. L. Olivares, J. Fleta et al., "Seasonality of cryptosporidiosis in children," European Journal of Clinical Microbiology \& Infectious Diseases, vol. 15, no. 1, pp. 77-79, 1996.

[54] J. Iqbal, P. R. Hira, F. Al-Ali, and R. Philip, "Cryptosporidiosis in Kuwaiti children: seasonality and endemicity," Clinical Microbiology and Infection, vol. 7, no. 5, pp. 261-266, 2001.

[55] J. S. Jagai, D. A. Castronovo, J. Monchak, and E. N. Naumova, "Seasonality of cryptosporidiosis: a meta-analysis approach," Environmental Research, vol. 109, no. 4, pp. 465-478, 2009.

[56] M. C. Peel, B. L. Finlayson, and T. A. McMahon, "Updated world map of the Köppen-Geiger climate classification," Hydrology and Earth System Sciences, vol. 11, no. 5, pp. 16331644, 2007.

[57] P. Das, S. S. Roy, K. MitraDhar et al., "Molecular characterization of Cryptosporidium spp. from children in Kolkata, India," Journal of Clinical Microbiology, vol. 44, no. 11, pp. 4246-4249, 2006.

[58] L. Savioli, H. Smith, and A. Thompson, "Giardia and Cryptosporidium join the 'neglected diseases initiative', Trends in Parasitology, vol. 22, no. 5, pp. 203-208, 2006.

[59] Neglected Diseases Initiative of the World Health Organization, http://www.who.int/neglected_diseases/en/.

[60] D. S. Berkman, A. G. Lescano, R. H. Gilman, S. L. Lopez, and M. M. Black, "Effects of stunting, diarrhoeal disease, and parasitic infection during infancy on cognition in late childhood: a follow-up study," The Lancet, vol. 359, no. 9306, pp. 564-571, 2002.

[61] R. Haque, D. Mondal, A. Karim et al., "Prospective casecontrol study of the association between common enteric protozoal parasites and diarrhea in Bangladesh," Clinical Infectious Diseases, vol. 48, no. 9, pp. 1191-1197, 2009.

[62] T. Geurden, B. Levecke, S. M. Cacciò et al., "Multilocus genotyping of Cryptosporidium and Giardia in non-outbreak related cases of diarrhoea in human patients in Belgium," Parasitology, vol. 136, no. 10, pp. 1161-1168, 2009.

[63] R. M. Chalmers, K. Elwin, A. L. Thomas, E. C. Guy, and B. Mason, "Long-term Cryptosporidium typing reveals the aetiology and species-specific epidemiology of human cryptosporidiosis in England and Wales, 2000 to 2003," Eurosurveillance, vol. 14, no. 2, Article ID 19086, 2009.

[64] P. H. P. Wong and C. S. L. Ong, "Molecular characterization of the Cryptosporidium cervine genotype," Parasitology, vol. 133, no. 6, pp. 693-700, 2006.

[65] G. Robinson, K. Elwin, and R. M. Chalmers, "Unusual Cryptosporidium genotypes in human cases of diarrhea," Emerging Infectious Diseases, vol. 14, no. 11, pp. 1800-1802, 2008.

[66] S. Tanriverdi, A. Grinberg, R. M. Chalmers et al., "Inferences about the global population structures of Cryptosporidium parvum and cryptosporidium hominis," Applied and Environmental Microbiology, vol. 74, no. 23, pp. 7227-7234, 2008.
[67] M. M. Peng, S. R. Meshnick, N. A. Cunliffe et al., "Molecular epidemiology of cryptosporidiosis in children in Malawi," The Journal of Eukaryotic Microbiology, vol. 50, supplement, pp. 557-559, 2003.

[68] W. Gatei, C. N. Wamae, C. Mbae et al., "Cryptosporidiosis: prevalence, genotype analysis, and symptoms associated with infections in children in Kenya," The American Journal of Tropical Medicine and Hygiene, vol. 75, no. 1, pp. 78-82, 2006.

[69] C. P. Raccurt, P. Brasseur, R. I. Verdier et al., "Human cryptosporidiosis and Cryptosporidium spp. in Haiti," Tropical Medicine \& International Health, vol. 11, no. 6, pp. 929-934, 2006.

[70] O. Y. Bushen, A. Kohli, R. C. Pinkerton et al., "Heavy cryptosporidial infections in children in northeast Brazil: comparison of Cryptosporidium hominis and Cryptosporidium parvum," Transactions of the Royal Society of Tropical Medicine and Hygiene, vol. 101, no. 4, pp. 378-384, 2007.

[71] A. Samie, P. O. Bessong, C. L. Obi et al., "Cryptosporidium species: preliminary descriptions of the prevalence and genotype distribution among school children and hospital patients in the Venda region, Limpopo Province, South Africa," Experimental Parasitology, vol. 114, no. 4, pp. 314322, 2006.

[72] B. A. Leav, M. R. Mackay, A. Anyanwu et al., "Analysis of sequence diversity at the highly polymorphic Cpgp40/15 locus among Cryptosporidium isolates from human immunodeficiency virus-infected children in South Africa," Infection and Immunity, vol. 70, no. 7, pp. 3881-3890, 2002.

[73] J. K. Tumwine, A. Kekitiinwa, S. Bakeera-Kitaka et al., "Cryptosporidiosis and microsporidiosis in Ugandan children with persistent diarrhea with and without concurrent infection with the human immunodeficiency virus," The American Journal of Tropical Medicine and Hygiene, vol. 73, no. 5, pp. 921-925, 2005.

[74] V. A. Cama, J. M. Ross, S. Crawford et al., "Differences in clinical manifestations among Cryptosporidium species and subtypes in HIV-infected persons," The Journal of Infectious Diseases, vol. 196, no. 5, pp. 684-691, 2007.

[75] O. Matos, M. Alves, L. Xiao, V. Cama, and F. Antunes, "Cryptosporidium felis and C. meleagridis in persons with HIV, Portugal," Emerging Infectious Diseases, vol. 10, no. 12, pp. 2256-2257, 2004.

[76] C.-C. Hung, J. C. Tsaihong, Y.-T. Lee et al., "Prevalence of intestinal infection due to Cryptosporidium species among Taiwanese patients with human immunodeficiency virus infection," Journal of the Formosan Medical Association, vol. 106, no. 1, pp. 31-35, 2007.

[77] A. R. Meamar, K. Guyot, G. Certad et al., "Molecular characterization of Cryptosporidium isolates from humans and animals in Iran," Applied and Environmental Microbiology, vol. 73, no. 3, pp. 1033-1035, 2007.

[78] M. Alves, L. Xiao, F. Antunes, and O. Matos, "Distribution of Cryptosporidium subtypes in humans and domestic and wild ruminants in Portugal," Parasitology Research, vol. 99, no. 3, pp. 287-292, 2006.

[79] R. M. Chalmers, C. Ferguson, S. M. Cacciò et al., "Direct comparison of selected methods for genetic categorisation of Cryptosporidium parvum and cryptosporidium hominis species," International Journal for Parasitology, vol. 35, no. 4, pp. 397-410, 2005.

[80] S. Glaberman, J. E. Moore, C. J. Lowery et al., "Three drinking-water-associated cryptosporidiosis outbreaks, Northern Ireland," Emerging Infectious Diseases, vol. 8, no. 6, pp. 631-633, 2002. 
[81] W. Smerdon, "Cryptosporidiosis outbreak associated with Majorcan hotel," Eurosurveillance, vol. 4, no. 34, Article ID 1540, 2000.

[82] L. Zhou, A. Singh, J. Jiang, and L. Xiao, "Molecular surveillance of Cryptosporidium spp. in raw wastewater in Milwaukee: implications for understanding outbreak occurrence and transmission dynamics," Journal of Clinical Microbiology, vol. 41, no. 11, pp. 5254-5257, 2003.

[83] W. Gatei, P. Das, P. Dutta et al., "Multilocus sequence typing and genetic structure of Cryptosporidium hominis from children in Kolkata, India," Infection, Genetics and Evolution, vol. 7, no. 2, pp. 197-205, 2007.

[84] S. Cohen, F. Dalle, A. Gallay, M. Di Palma, A. Bonnin, and H. D. Ward, "Identification of Cpgp40/15 type Ib as the predominant allele in isolates of Cryptosporidium spp. from a waterborne outbreak of gastroenteritis in South Burgundy, France," Journal of Clinical Microbiology, vol. 44, no. 2, pp. 589-591, 2006.

[85] T. K. Boehmer, N. B. Alden, T. S. Ghosh, and R. L. Vogt, "Cryptosporidiosis from a community swimming pool: outbreak investigation and follow-up study," Epidemiology and Infection, vol. 137, no. 11, pp. 1651-1654, 2009.

[86] C. S. Ong, S. Chow, P. P. L. So, et al., "Identification of two different cryptosporidium hominis subtypes from cases in the 2001 waterborne cryptosporidiosis outbreak in North Battleford, Saskatchewan," in Proceedings of the 11th Canadian National Conference and the 2nd Policy Forum on Drinking Water, Calgary, Canada, April 2004, pp. 628638, Canadian Water and Wastewater Association, Ottawa, Canada, 2005.

[87] L. Xiao, R. Fayer, U. Ryan, and S. J. Upton, “Cryptosporidium taxonomy: recent advances and implications for public health," Clinical Microbiology Reviews, vol. 17, no. 1, pp. 7297, 2004.

[88] W. Gatei, D. Barrett, J. F. Lindo, D. Eldemire-Shearer, V. Cama, and L. Xiao, "Unique Cryptosporidium population in HIV-infected persons, Jamaica," Emerging Infectious Diseases, vol. 14, no. 5, pp. 841-843, 2008.

[89] D. Muthusamy, S. S. Rao, S. Ramani et al., "Multilocus genotyping of Cryptosporidium sp. isolates from human immunodeficiency virus-infected individuals in South India," Journal of Clinical Microbiology, vol. 44, no. 2, pp. 632-634, 2006.

[90] A. R. Jex and R. B. Gasser, "Genetic richness and diversity in cryptosporidium hominis and C. parvum reveals major knowledge gaps and a need for the application of "next generation" technologies—research review," Biotechnology Advances, vol. 28, no. 1, pp. 17-26, 2010.

[91] J. Dreesman, D. C. Villarroel-Conzales, S. Cleves, H. A. Reins, and M. Pulz, "Regionally increased incidence of notified cryptosporidiosis cases due to different laboratory methods," Gesundheitswesen, vol. 69, no. 8-9, pp. 483-487, 2007.

[92] J. S. Yoder and M. J. Beach, "Cryptosporidiosis surveillanceUnited States, 2003-2005," Morbidity and Mortality Weekly Report, vol. 56, no. 7, pp. 1-10, 2007.

[93] Center for Disease Control and Prevention, http://emergency .cdc.gov/agent/agentlist-category.asp.

[94] J. M. Stuart, H. J. Orr, F. G. Warburton et al., "Risk factors for sporadic giardiasis: a case-control study in Southwestern England," Emerging Infectious Diseases, vol. 9, no. 2, pp. 229233, 2003.

[95] S. L. Roy, S. M. DeLong, S. A. Stenzel et al., "Risk factors for sporadic cryptosporidiosis among immunocompetent persons in the United States from 1999 to 2001," Journal of Clinical Microbiology, vol. 42, no. 7, pp. 2944-2951, 2004.
[96] S. Coupe, K. Delabre, R. Pouillot, S. Houdart, M. SantillanaHayat, and F. Derouin, "Detection of Cryptosporidium, Giardia and Enterocytozoon bieneusi in surface water, including recreational areas: a one-year prospective study," FEMS Immunology and Medical Microbiology, vol. 47, no. 3, pp. 351-359, 2006.

[97] R. Fayer, "Cryptosporidium: a water-borne zoonotic parasite," Veterinary Parasitology, vol. 126, no. 1-2, pp. 37-56, 2004.

[98] H. Pelly, M. Cormican, D. O’Donovan et al., "A large outbreak of cryptosporidiosis in western Ireland linked to public water supply: a preliminary report," Eurosurveillance, vol. 12, no. 5, Article ID 3187, 2007.

[99] P. Jennings and A. Rhatigan, "Cryptosporidiosis outbreak in Ireland linked to public water supply," Eurosurveillance, vol. 6, no. 22, Article ID 2089, 2002.

[100] H.-W. A. Cheng, F. E. Lucy, T. K. Graczyk, M. A. Broaders, L. Tamang, and M. Connolly, "Fate of Cryptosporidium parvum and Cryptosporidium hominis oocysts and Giardia duodenalis cysts during secondary wastewater treatments," Parasitology Research, vol. 105, no. 3, pp. 689-696, 2009.

[101] H. P. Thompson, J. S. G. Dooley, J. Kenny et al., "Genotypes and subtypes of Cryptosporidium spp. in neonatal calves in Northern Ireland," Parasitology Research, vol. 100, no. 3, pp. 619-624, 2007.

[102] F. M. Schets, J. H. van Wijnen, J. F. Schijven, H. Schoon, and A. M. de Roda Husman, "Monitoring of waterborne pathogens in surface waters in Amsterdam, the Netherlands, and the potential health risk associated with exposure to Cryptosporidium and Giardia in these waters," Applied and Environmental Microbiology, vol. 74, no. 7, pp. 2069-2078, 2008.

[103] M. L. Lobo, L. Xiao, F. Antunes, and O. Matos, "Occurrence of Cryptosporidium and Giardia genotypes and subtypes in raw and treated water in Portugal," Letters in Applied Microbiology, vol. 48, no. 6, pp. 732-737, 2009.

[104] M. Alves, L. Xiao, I. Sulaiman, A. A. Lal, O. Matos, and F. Antunes, "Subgenotype analysis of Cryptosporidium isolates from humans, cattle, and zoo ruminants in Portugal," Journal of Clinical Microbiology, vol. 41, no. 6, pp. 2744-2747, 2003.

[105] L. J. Robertson, L. Hermansen, and B. K. Gjerde, "Occurrence of Cryptosporidium oocysts and Giardia cysts in sewage in Norway," Applied and Environmental Microbiology, vol. 72, no. 8, pp. 5297-5303, 2006.

[106] T. Geurden, E. Goossens, B. Levecke, F. Vercammen, J. Vercruysse, and E. Claerebout, "Occurrence and molecular characterization of Cryptosporidium and Giardia in captive wild ruminants in Belgium," Journal of Zoo and Wildlife Medicine, vol. 40, no. 1, pp. 126-130, 2009.

[107] N. A. Romanenko, V. P. Sergiev, and I. A. Rakhmanin, “Cryptosporidium oocysts and epidemic safety of drinking water in the Russian Federation," Meditsinskaya Parazitologiya $i$ Parazitarnye Bolezni, no. 2, pp. 11-13, 2001.

[108] A. Giangaspero, "Giardia, Cryptosporidium and the spectre of zoonosis: the Italian experience from land to sea," Parassitologia, vol. 48, no. 1-2, pp. 95-100, 2006.

[109] A. Giangaspero, F. Berrilli, and O. Brandonisio, "Giardia and Cryptosporidium and public health: the epidemiological scenario from the Italian perspective," Parasitology Research, vol. 101, no. 5, pp. 1169-1182, 2007.

[110] O. Brandonisio, "Waterborne transmission of Giardia and Cryptosporidium," Parassitologia, vol. 48, no. 1-2, pp. 91-94, 2006. 
[111] O. Brandonisio, L. Fumarola, R. Spinelli, F. Donadio, P. Montemurro, and F. Portincasa, "Giardia e Cryptosporidium spp: rassegna critica e monitoraggio in acque superficiali e reflue," L'igiene Moderna, vol. 122, pp. 137-160, 2004.

[112] R. Briancesco and L. Bonadonna, "An Italian study on Cryptosporidium and Giardia in wastewater, fresh water and treated water," Environmental Monitoring and Assessment, vol. 104, no. 1-3, pp. 445-457, 2005.

[113] M. A. Di Benedetto, F. Di Piazza, C. M. Maida, A. Firenze, and R. Oliveri, "Occurrence of Giardia and Cryptosporidium in wastewater, surface water and ground water samples in Palermo (Sicily)," Annali di Igiene, vol. 17, no. 5, pp. 367-375, 2005.

[114] C. Sacco, M. Bianchi, C. Lorini, D. Burrini, S. Berchielli, and E. Lanciotti, "Removal of Cryptosporidium and Giardia in drinking water treatment in a Tuscan area," Annali di Igiene, vol. 18, no. 2, pp. 117-126, 2006.

[115] A. Lonigro, A. Pollice, R. Spinelli et al., "Giardia cysts and Cryptosporidium oocysts in membrane-filtered municipal wastewater used for irrigation," Applied and Environmental Microbiology, vol. 72, no. 12, pp. 7916-7918, 2006.

[116] E. Carraro, E. Fea, S. Salva, and G. Gilli, "Impact of a wastewater treatment plant on Cryptosporidium oocysts and Giardia cysts occurring in a surface water," Water Science and Technology, vol. 41, no. 7, pp. 31-37, 2000.

[117] S. M. Cacciò, M. De Giacomo, F. A. Aulicino, and E. Pozio, "Giardia cysts in wastewater treatment plants in Italy," Applied and Environmental Microbiology, vol. 69, no. 6, pp. 3393-3398, 2003.

[118] R. Fayer, J. P. Dubey, and D. S. Lindsay, "Zoonotic protozoa: from land to sea," Trends in Parasitology, vol. 20, no. 11, pp. 531-536, 2004.

[119] A. Giangaspero, U. Molini, R. Iorio, D. Traversa, B. Paoletti, and C. Giansante, "Cryptosporidium parvum oocysts in seawater clams (Chamelea gallina) in Italy," Preventive Veterinary Medicine, vol. 69, no. 3-4, pp. 203-212, 2005.

[120] U. Molini, D. Traversa, G. Ceschia et al., "Temporal occurrence of Cryptosporidium in the Manila clam Ruditapes philippinarum in Northern Adriatic Italian Lagoons," Journal of Food Protection, vol. 70, no. 2, pp. 494-499, 2007.

[121] A. Giangaspero, R. Cirillo, V. Lacasella et al., "Giardia and Cryptosporidium in inflowing water and harvested shellfish in a Lagoon in Southern Italy," Parasitology International, vol. 58 , no. 1, pp. 12-17, 2009.

[122] W. A. Miller, M. A. Miller, I. A. Gardner et al., "New genotypes and factors associated with Cryptosporidium detection in mussels (Mytilus spp.) along the California coast," International Journal for Parasitology, vol. 35, no. 10, pp. 1103-1113, 2005.

[123] B. Paoletti, A. Giangaspero, A. Gatti et al., "Immunoenzymatic analysis and genetic detection of Cryptosporidium parvum in lambs from Italy," Experimental Parasitology, vol. 122, no. 4, pp. 349-352, 2009.

[124] A. Duranti, S. M. Cacciò, E. Pozio et al., "Risk factors associated with Cryptosporidium parvum infection in cattle," Zoonoses and Public Health, vol. 56, no. 4, pp. 176-182, 2009.

[125] L. A. Trotz-Williams, S. W. Martin, K. E. Leslie, T. Duffield, D. V. Nydam, and A. S. Peregrine, "Association between management practices and within-herd prevalence of Cryptosporidium parvum shedding on dairy farms in southern Ontario," Preventive Veterinary Medicine, vol. 83, no. 1, pp. 11-23, 2008.
[126] N. Bilenko, R. Ghosh, A. Levy, R. J. Deckelbaum, and O. Fraser, "Partial breastfeeding protects Bedouin infants from infection and morbidity: prospective cohort study," Asia Pacific Journal of Clinical Nutrition, vol. 17, no. 2, pp. 243249, 2008.

[127] C. Wheeler, D. J. Vugia, G. Thomas et al., "Outbreak of cryptosporidiosis at a California waterpark: employee and patron roles and the long road towards prevention," Epidemiology and Infection, vol. 135, no. 2, pp. 302-310, 2007.

[128] F. J. Sorvillo, K. Fujioka, B. Nahlen, M. P. Tormey, R. Kebabjian, and L. Mascola, "Swimming-associated cryptosporidiosis," American Journal of Public Health, vol. 82, no. 5, pp. 742-744, 1992.

[129] A. L. Valderrama, M. C. Hlavsa, A. Cronquist et al., "Multiple risk factors associated with a large statewide increase in cryptosporidiosis," Epidemiology and Infection, vol. 137, no. 12, pp. 1781-1788, 2009.

[130] G. F. Craun, R. L. Calderon, and M. F. Craun, "Outbreaks associated with recreational water in the United States," International Journal of Environmental Health Research, vol. 15, no. 4, pp. 243-262, 2005.

[131] J. S. Yoder, M. C. Hlavsa, G. F. Craun et al., "Surveillance for waterborne disease and outbreaks associated with recreational water use and other aquatic facility-associated health events-United States, 2005-2006," Morbidity and Mortality Weekly Report, vol. 57, no. 9, pp. 1-29, 2008.

[132] B. G. Blackburn, G. F. Craun, J. S. Yoder et al., "Surveillance for waterborne-disease outbreaks associated with drinking water-United States, 2001-2002," Morbidity and Mortality Weekly Report, vol. 53, no. 8, pp. 23-45, 2004.

[133] R. L. Calderon and G. F. Craun, "Estimates of endemic waterborne risks from community-intervention studies," Journal of Water and Health, vol. 4, no. 2, pp. 89-100, 2006.

[134] G. F. Craun, N. Nwachuku, R. L. Calderon, and M. F. Craun, “Outbreaks in drinking-water systems, 1991-1998," Journal of Environmental Health, vol. 65, no. 1, pp. 16-23, 2002.

[135] A. Khalakdina, D. J. Vugia, J. Nadle, G. A. Rothrock, and J. M. Colford Jr., "Is drinking water a risk factor for endemic cryptosporidiosis? A case-control study in the immunocompetent general population of the San Francisco Bay Area," BMC Public Health, vol. 3, no. 1, article 11, 2003.

[136] San Francisco Bay Area Cryptosporidiosis Surveillance Project, http://www.sfphes.org/water/index_crypto.htm.

[137] K. A. Reynolds, K. D. Mena, and C. P. Gerba, "Risk of waterborne illness via drinking water in the United States," Reviews of Environmental Contamination and Toxicology, vol. 192, pp. 117-158, 2008.

[138] P. Payment, E. Franco, L. Richardson, and J. Siemiatycki, "Gastrointestinal health effects associated with the consumption of drinking water produced by point-of-use domestic reverse-osmosis filtration units," Applied and Environmental Microbiology, vol. 57, no. 4, pp. 945-948, 1991.

[139] P. Payment, A. Berte, and C. Fleury, "Sources of variation in isolation rate of Giardia lamblia cysts and their homogeneous distribution in river water entering a water treatment plant," Canadian Journal of Microbiology, vol. 43, no. 7, pp. 687-689, 1997.

[140] M. E. Hellard, M. I. Sinclair, A. B. Forbes, and C. K. Fairley, "A randomized, blinded, controlled trial investigating the gastrointestinal health effects of drinking water quality," Environmental Health Perspectives, vol. 109, no. 8, pp. 773778, 2001. 
[141] J. M. Colford Jr., T. J. Wade, K. C. Schiff et al., "Water quality indicators and the risk of illness at beaches with nonpoint sources of fecal contamination," Epidemiology, vol. 18, no. 1, pp. 27-35, 2007.

[142] R. D. Arnone and J. P. Walling, "Evaluating Cryptosporidium and Giardia concentrations in combined sewer overflow," Journal of Water and Health, vol. 4, no. 2, pp. 157-165, 2006.

[143] M. M. Peng, L. Xiao, A. R. Freeman et al., "Genetic polymorphism among Cryptosporidium parvum isolates: evidence of two distinct human transmission cycles," Emerging Infectious Diseases, vol. 3, no. 4, pp. 567-573, 1997.

[144] I. M. Sulaiman, L. Xiao, C. Yang et al., "Differentiating human from animal isolates of Cryptosporidium parvum," Emerging Infectious Diseases, vol. 4, no. 4, pp. 681-685, 1998.

[145] V. Dietz, D. Vugia, R. Nelson et al., "Active, multisite, laboratory-based surveillance for Cryptosporidium parvum," The American Journal of Tropical Medicine and Hygiene, vol. 62, no. 3, pp. 368-372, 2000.

[146] M. C. Hlavsa, J. C. Watson, and M. J. Beach, "Cryptosporidiosis surveillance-United States 1999-2002," Morbidity and Mortality Weekly Report, vol. 54, no. 1, pp. 1-8, 2005.

[147] W. R. MacKenzie, N. J. Hoxie, M. E. Proctor et al., "A massive outbreak in Milwaukee of Cryptosporidium infection transmitted through the public water supply," The New England Journal of Medicine, vol. 331, no. 3, pp. 161-167, 1994.

[148] D. C. Feltus, C. W. Giddings, B. L. Schneck, T. Monson, D. Warshauer, and J. M. McEvoy, "Evidence supporting zoonotic transmission of Cryptosporidium spp. in Wisconsin," Journal of Clinical Microbiology, vol. 44, no. 12, pp. 4303-4308, 2006.

[149] J. Jiang, K. A. Alderisio, and L. Xiao, "Distribution of Cryptosporidium genotypes in storm event water samples from three watersheds in New York," Applied and Environmental Microbiology, vol. 71, no. 8, pp. 4446-4454, 2005.

[150] Tri-County Health Department, http://www.tchd.org/.

[151] S. M. Dorner, W. B. Anderson, T. Gaulin et al., "Pathogen and indicator variability in a heavily impacted watershed," Journal of Water and Health, vol. 5, no. 2, pp. 241-257, 2007.

[152] K. Jaidi, B. Barbeau, A. Carrière, R. Desjardins, and M. Prévost, "Including operational data in QMRA model: development and impact of model inputs," Journal of Water and Health, vol. 7, no. 1, pp. 77-95, 2009.

[153] Public Health Agency of Canada, http://www.phac-aspc.gc .ca/index-eng.php.

[154] C-EnterNet (Canadian Integrated Enteric Disease Surveillance System), http://www.phac-aspc.gc.ca/c-enternet/ index-eng.php.

[155] K. D.M. Pintar, F. Pollari, D. Waltner-Toews et al., "A modified case-control study of cryptosporidiosis (using nonCryptosporidium-infected enteric cases as controls) in a community setting," Epidemiology and Infection, vol. 137, no. 12, pp. 1789-1799, 2009.

[156] E. J. Dziuban, J. L. Liang, G. F. Craun et al., "Surveillance for waterborne disease and outbreaks associated with recreational water-United States, 2003-2004," Morbidity and Mortality Weekly Report, vol. 55, no. 12, pp. 1-30, 2006.

[157] S. J. Snel, M. G. Baker, and K. Venugopal, "The epidemiology of cryptosporidiosis in New Zealand, 1997-2006," The New Zealand Medical Journal, vol. 122, no. 1290, pp. 47-61, 2009.
[158] J. J. Learmonth, G. Ionas, K. A. Ebbett, and E. S. Kwan, "Genetic characterization and transmission cycles of Cryptosporidium species isolated from humans in New Zealand," Applied and Environmental Microbiology, vol. 70, no. 7, pp. 3973-3978, 2004.

[159] J. McLauchlin, C. Amar, S. Pedraza-Díaz, and G. L. Nichols, "Molecular epidemiological analysis of Cryptosporidium spp. in the United kingdom: results of genotyping Cryptosporidium spp. in 1,705 fecal samples from humans and 105 fecal samples from livestock animals," Journal of Clinical Microbiology, vol. 38, no. 11, pp. 3984-3990, 2000.

[160] L. S. Waldron, B. C. Ferrari, and M. L. Power, "Glycoprotein 60 diversity in C. hominis and C. parvum causing human cryptosporidiosis in NSW, Australia," Experimental Parasitology, vol. 122, no. 2, pp. 124-127, 2009.

[161] AIDS, http://www.unaids.org/en/default.asp.

[162] A. A. Gideon, T. Njiné, M. Nola, S. F. Menbohan, and M. W. Ndayo, "Measuring resistant forms of two pathogenic protozoa (Giardia spp and Cryptosporidium spp) in two aquatic biotopes in Yaoundé (Cameroon)," Cahiers Sante, vol. 17, no. 3, pp. 167-172, 2007.

[163] F. G. Youssef, I. Adib, M. S. Riddle, and C. D. Schlett, "A review of cryptosporidiosis in Egypt," Journal of the Egyptian Society of Parasitology, vol. 38, no. 1, pp. 9-28, 2008.

[164] M. Pirestani, J. Sadraei, A. Dalimi Asl, M. Zavvar, and H. Vaeznia, "Molecular characterization of Cryptosporidium isolates from human and bovine using 18s rRNA gene in Shahriar county of Tehran, Iran," Parasitology Research, vol. 103, no. 2, pp. 467-472, 2008.

[165] A. Liu, R. Wang, Y. Li et al., "Prevalence and distribution of Cryptosporidium spp. in dairy cattle in Heilongjiang Province, China," Parasitology Research, vol. 105, no. 3, pp. 797-802, 2009.

[166] R. Fayer, M. Santín, and J. M. Trout, "Cryptosporidium ryanae n. sp. (Apicomplexa: Cryptosporidiidae) in cattle (Bos taurus)," Veterinary Parasitology, vol. 156, no. 3-4, pp. 191198, 2008.

[167] Y. Feng, Y. Ortega, G. He et al., "Wide geographic distribution of Cryptosporidium bovis and the deer-like genotype in bovines," Veterinary Parasitology, vol. 144, no. 1-2, pp. 1-9, 2007.

[168] B.-M. Hsu, C. Huang, G.-Y. Jiang, and C.-L. L. Hsu, “The prevalence of Giardia and Cryptosporidium in Taiwan water supplies," Journal of Toxicology and Environmental Health Part A, vol. 57, no. 3, pp. 149-160, 1999.

[169] S. Paul, D. Chandra, D. D. Ray et al., "Prevalence and molecular characterization of bovine Cryptosporidium isolates in India," Veterinary Parasitology, vol. 153, no. 1-2, pp. 143-146, 2008.

[170] S. Paul, D. Chandra, A. K. Tewari et al., "Prevalence of Cryptosporidium andersoni: a molecular epidemiological survey among cattle in India," Veterinary Parasitology, vol. 161, no. 1-2, pp. 31-35, 2009.

[171] S. S. Roy, S. Sarkar, S. Batabyal, A. K. Pramanik, and P. Das, "Observations on the epidemiology of bovine cryptosporidiosis in India," Veterinary Parasitology, vol. 141, no. 3-4, pp. 330-333, 2006.

[172] R. Fayer, U. Morgan, and S. J. Upton, "Epidemiology of Cryptosporidium: transmission, detection and identification," International Journal for Parasitology, vol. 30, no. 12-13, pp. 1305-1322, 2000. 
[173] S. Goh, M. Reacher, D. P. Casemore et al., "Sporadic cryptosporidiosis, North Cumbria, England, 1996-2000," Emerging Infectious Diseases, vol. 10, no. 6, pp. 1007-1015, 2004.

[174] M. C. A. Teixeira, M. L. Barreto, C. Melo, L. R. Silva, L. R. S. Moraes, and N. M. Alcântara-Neves, "A serological study of Cryptosporidium transmission in a periurban area of a Brazilian Northeastern city," Tropical Medicine \& International Health, vol. 12, no. 9, pp. 1096-1104, 2007.

[175] M. U. Alonso-Fresán, J. C. Vázquez-Chagoyán, V. VelázquezOrdoñez, N. Pescador-Salas, and J. Saltijeral-Oaxaca, "Sheep management and cryptosporidiosis in central Mexico," Tropical Animal Health and Production, vol. 41, no. 4, pp. 431436, 2009.

[176] E. C.D. Todd, J. D. Greig, C. A. Bartleson, and B. S. Michaels, "Outbreaks where food workers have been implicated in the spread of foodborne disease. Part 6. Transmission and survival of pathogens in the food processing and preparation environment," Journal of Food Protection, vol. 72, no. 1, pp. 202-219, 2009.

[177] E. Pozio, "Epidemiology and control prospects of foodborne parasitic zoonoses in the European Union," Parassitologia, vol. 50, no. 1-2, pp. 17-24, 2008.

[178] A. Pönka, P. Kotilainen, R. Rimhanen-Finne et al., "A foodborne outbreak due to Cryptosporidium parvum in Helsinki, November 2008," Eurosurveillance, vol. 14, no. 28, Article ID 19269, 2009.

[179] M. Insulander, B. de Jong, and B. Svenungsson, "A foodborne outbreak of cryptosporidiosis among guests and staff at a hotel restaurant in Stockholm county, Sweden, September 2008," Eurosurveillance, vol. 13, no. 51, Article ID 19071, 2008.

[180] S. Ethelberg, M. Lisby, L. S. Vestergaard et al., "A foodborne outbreak of Cryptosporidium hominis infection," Epidemiology and Infection, vol. 137, no. 3, pp. 348-356, 2009.

[181] P. S. Millard, K. F. Gensheimer, D. G. Addiss et al., "An outbreak of cryptosporidiosis from fresh-pressed apple cider," Journal of the American Medical Association, vol. 272, no. 20, pp. 1592-1596, 1994.

[182] B. G. Blackburn, J. M. Mazurek, M. Hlavsa et al., "Cryptosporidiosis associated with ozonated apple cider," Emerging Infectious Diseases, vol. 12, no. 4, pp. 684-686, 2006.

[183] FoodNet, http://www.cdc.gov/FoodNet/.

[184] J. D. Vojdani, L. R. Beuchat, and R. V. Tauxe, "Juiceassociated outbreaks of human illness in the United States, 1995 through 2005," Journal of Food Protection, vol. 71, no. 2, pp. 356-364, 2008.

[185] M. Lynch, J. Painter, R. Woodruff, and C. Braden, "Surveillance for foodborne-disease outbreaks-United States, 19982002," Morbidity and Mortality Weekly Report, vol. 55, no. 10, pp. 1-42, 2006.

[186] Centers for Disease Control and Prevention (CDC), "Preliminary FoodNet Data on the incidence of infection with pathogens transmitted commonly through food-10 States, United States 2003," Morbidity and Mortality Weekly Report, vol. 53, no. 16, pp. 338-343, 2004.

[187] Centers for Disease Control and Prevention (CDC), "Preliminary FoodNet Data on the incidence of infection with pathogens transmitted commonly through food-10 States, United States 2004"', Morbidity and Mortality Weekly Report, vol. 54, no. 14, pp. 352-356, 2005.
[188] D. Vugia, A. Cronquist, J. Hadler et al., "Preliminary FoodNet data on the incidence of infection with pathogens transmitted commonly through food-10 States, United States, 2005," Morbidity and Mortality Weekly Report, vol. 55, no. 14, pp. 392-393, 2006.

[189] D. Vugia, A. Cronquist, J. Hadler et al., "Preliminary FoodNet data on the incidence of infection with pathogens transmitted commonly through food-10 States, 2006," Morbidity and Mortality Weekly Report, vol. 56, no. 14, pp. 336-339, 2007.

[190] D. Vugia, A. Cronquist, J. Hadler et al., "Preliminary FoodNet data on the incidence of infection with pathogens transmitted commonly through food-10 States, 2007," Morbidity and Mortality Weekly Report, vol. 57, no. 14, pp. 366-370, 2008.

[191] Centers for Disease Control and Prevention (CDC), "Preliminary FoodNet Data on the incidence of infection with pathogens transmitted commonly through food-10 States, United States 2008," Morbidity and Mortality Weekly Report, vol. 58, no. 13, pp. 333-337, 2009.

[192] A. D. Freites-Martínez, D. Colmenares, M. Pérez, M. García, and O. Díaz de Suárez, "Cryptosporidium sp infections and other intestinal parasites in food handlers from Zulia state, Venezuela," Investigacion Clinica, vol. 50, no. 1, pp. 13-21, 2009.

[193] M. Calvo, M. Carazo, M. L. Arias, C. Chaves, R. Monge, and M. Chinchilla, "Prevalence of Cyclospora sp., Cryptosporidium sp, microsporidia and fecal coliform determination in fresh fruit and vegetables consumed in Costa Rica," Archivos Latinoamericanos de Nutrición, vol. 54, no. 4, pp. 428-432, 2004.

[194] J.-D. Cavallo and E. Garrabé, "Infectious aetiologies of travelers' diarrhoea," Médecine et Maladies Infectieuses, vol. 37, no. 11, pp. 722-727, 2007.

[195] L. Saiman, J. Aronson, J. Zhou et al., "Prevalence of infectious diseases among internationally adopted children," Pediatrics, vol. 108, no. 3, pp. 608-612, 2001.

[196] D. O. Freedman, L. H. Weld, P. E. Kozarsky et al., "Spectrum of disease and relation to place of exposure among ill returned travelers," The New England Journal of Medicine, vol. 354, no. 2, pp. 119-130, 2006.

[197] S. Ansart, L. Perez, O. Vergely, M. Danis, F. Bricaire, and E. Caumes, "Illnesses in travelers returning from the tropics: a prospective study of 622 patients," Journal of Travel Medicine, vol. 12, no. 6, pp. 312-318, 2005.

[198] S. R. Caruana, H. A. Kelly, J. Y. Y. Ngeow et al., "Undiagnosed and potentially lethal parasite infections among immigrants and refugees in Australia," Journal of Travel Medicine, vol. 13, no. 4, pp. 233-239, 2006.

[199] C. J. M. Whitty, B. Carroll, M. Armstrong et al., "Utility of history, examination and laboratory tests in screening those returning to Europe from the tropics for parasitic infection," Tropical Medicine \& International Health, vol. 5, no. 11, pp. 818-823, 2000.

[200] R. Fotedar, D. Stark, N. Beebe, D. Marriott, J. Ellis, and J. Harkness, "Laboratory diagnostic techniques for Entamoeba species," Clinical Microbiology Reviews, vol. 20, no. 3, pp. 511-532, 2007.

[201] R. Goodgame, "Emerging causes of traveler's diarrhea: Cryptosporidium, Cyclospora, Isospora, and Microsporidia," Current Infectious Disease Reports, vol. 5, no. 1, pp. 66-73, 2003.

[202] M. A. S. de Wit, M. P. G. Koopmans, L. M. Kortbeek et al., "Sensor, a population-based cohort study on gastroenteritis in the Netherlands: incidence and etiology," American Journal of Epidemiology, vol. 154, no. 7, pp. 666-674, 2001. 
[203] N. M. Thielman and R. L. Guerrant, "Persistent diarrhea in the returned traveler," Infectious Disease Clinics of North America, vol. 12, no. 2, pp. 489-501, 1998.

[204] P. C. Okhuysen, "Traveler's diarrhea due to intestinal protozoa," Clinical Infectious Diseases, vol. 33, no. 1, pp. 110-114, 2001.

[205] N. Turgay, A. Yolasigmaz, D. D. Erdoğan, F. Y. Zeyrek, and A. Uner, "Incidence of cyclosporiasis in patients with gastrointestinal symptoms in western Turkey," Medical Science Monitor, vol. 13, no. 1, pp. CR34-CR39, 2007.

[206] P. Nair, J. A. Mohamed, H. L. DuPont et al., "Epidemiology of cryptosporidiosis in north American travelers to Mexico," The American Journal of Tropical Medicine and Hygiene, vol. 79, no. 2, pp. 210-214, 2008.

[207] R. Lazensky, R. M. Hammond, K. Van Zile, and K. Geib, "Cryptosporidiosis outbreak in a Nassau County, Florida, return travel group from Ireland, may 24, 2006-june 4, 2006," Journal of Environmental Health, vol. 71, no. 2, pp. 20-24, 2008.

[208] R. B. Gasser, Y. G. Abs EL-Osta, and R. M. Chalmers, "Electrophoretic analysis of genetic variability within Cryptosporidium parvum from imported and autochthonous cases of human cryptosporidiosis in the United Kingdom," Applied and Environmental Microbiology, vol. 69, no. 5, pp. 27192730, 2003.

[209] R. M. Chalmers, S. J. Hadfield, C. J. Jackson, K. Elwin, L. Xiao, and P. Hunter, "Geographic linkage and variation in Cryptosporidium hominis," Emerging Infectious Diseases, vol. 14, no. 3, pp. 496-498, 2008.

[210] T. Weitzel, S. Dittrich, I. Möhl, E. Adusu, and T. Jelinek, "Evaluation of seven commercial antigen detection tests for Giardia and Cryptosporidium in stool samples," Clinical Microbiology and Infection, vol. 12, no. 7, pp. 656-659, 2006.

[211] M. T. Katanik, S. K. Schneider, J. E. Rosenblatt, G. S. Hall, and G. W. Procop, "Evaluation of ColorPAC Giardia/Cryptosporidium rapid assay and ProSpect Giardia/Cryptosporidium microplate assay for detection of Giardia and Cryptosporidium in fecal specimens," Journal of Clinical Microbiology, vol. 39, no. 12, pp. 4523-4525, 2001.

[212] L. S. Garcia and R. Y. Shimizu, "Evaluation of nine immunoassay kits (enzyme immunoassay and direct fluorescence) for detection of Giardia lamblia and Cryptosporidium parvum in human fecal specimens," Journal of Clinical Microbiology, vol. 35, no. 6, pp. 1526-1529, 1997.

[213] R. J. Ten Hove, M. van Esbroeck, T. Vervoort, J. van den Ende, L. van Lieshout, and J. J. Verweij, "Molecular diagnostics of intestinal parasites in returning travellers," European Journal of Clinical Microbiology \& Infectious Diseases, vol. 28, no. 9, pp. 1045-1053, 2009.

[214] H. Rotterdam and P. Tsang, "Gastrointestinal disease in the immunocompromised patient," Human Pathology, vol. 25, no. 11, pp. 1123-1140, 1994.

[215] R. Weber, B. Ledergerber, R. Zbinden et al., "Enteric infections and diarrhea in human virus-infected persons: prospective community-based cohort study," Archives of Internal Medicine, vol. 159, no. 13, pp. 1473-1480, 1999.

[216] J. A. Montero, J. T. Sinnott, D. A. Holt, and C. Lloyd, "Biliary cryptosporidiosis: current concepts," Infections in Medicine, vol. 18 , no. 6, pp. 312-316, 2001.

[217] M. Scaglia, S. Gatti, P. Bassi, P. L. Viale, S. Novati, and S. Ranieri, "Intestinal co-infection by Cyclospora sp. and Cryptosporidium parvum: first report in an AIDS patient," Parasite, vol. 1, no. 4, pp. 387-390, 1994.
[218] D. Reijasse, N. Patey-Mariaud de Serre, D. Canioni et al., "Cytotoxic T cells in AIDS colonic cryptosporidiosis," Journal of Clinical Pathology, vol. 54, no. 4, pp. 298-303, 2001.

[219] Y. M. Miao, F. M. Awad-El-Kariem, C. Franzen et al., "Eradication of cryptosporidia and microsporidia following successful antiretroviral therapy," Journal of Acquired Immune Deficiency Syndromes, vol. 25, no. 2, pp. 124-129, 2000.

[220] N. A. Foudraine, G. J. Weverling, T. van Cool et al., "Improvement of chronic diarrhoea in patients with advanced HIV-1 infection during potent antiretroviral therapy," AIDS, vol. 12, no. 1, pp. 35-41, 1998.

[221] E. Pozio and M. A. G. Morales, "The impact of HIV-protease inhibitors on opportunistic parasites," Trends in Parasitology, vol. 21, no. 2, pp. 58-63, 2005.

[222] W. Gatei, J. Greensill, R. W. Ashford et al., "Molecular analysis of the 18S rRNA gene of Cryptosporidium parasites from patients with or without human immunodeficiency virus infections living in Kenya, Malawi, Brazil, the United Kingdom, and Vietnam," Journal of Clinical Microbiology, vol. 41, no. 4, pp. 1458-1462, 2003.

[223] U. Morgan, R. Weber, L. Xiao et al., "Molecular characterization of Cryptosporidium isolates obtained from human immunodeficiency virus-infected individuals living in Switzerland, Kenya, and the United States," Journal of Clinical Microbiology, vol. 38, no. 3, pp. 1180-1183, 2000.

[224] M. T. Llorente, A. Clavel, M. P. Goñi et al., "Genetic characterization of Cryptosporidium species from humans in Spain," Parasitology International, vol. 56, no. 3, pp. 201-205, 2007.

[225] O. Brandonisio, P. Maggi, M. A. Panaro, L. A. Bramante, A. Di Coste, and G. Angarano, "Prevalence of cryptosporidiosis in HIV-infected patients with diarrhoeal illness," European Journal of Epidemiology, vol. 9, no. 2, pp. 190-194, 1993.

[226] E. Pozio, G. Rezza, A. Boschini et al., "Clinical cryptosporidiosis and human immunodeficiency virus (HIV)-induced immunosuppression: findings from a longitudinal study of HIV-positive and HIV-negative former injection drug users," The Journal of Infectious Diseases, vol. 176, no. 4, pp. 969-975, 1997.

[227] P. Rossi, F. Rivasi, M. Codeluppi et al., "Gastric involvement in AIDS associated cryptosporidiosis," Gut, vol. 43, no. 4, pp. 476-477, 1998.

[228] O. Brandonisio, P. Maggi, M. A. Panaro et al., "Intestinal protozoa in HIV-infected patients in Apulia, South Italy," Epidemiology and Infection, vol. 123, no. 3, pp. 457-462, 1999.

[229] D. Dionisio, "Cryptosporidiosis in HIV-infected patients," Journal of Postgraduate Medicine, vol. 48, no. 3, pp. 215-216, 2002.

[230] C. C. McOliver, H. B. Lemerman, E. K. Silbergeld, R. D. Moore, and T. K. Graczyk, "Risks of recreational exposure to waterborne pathogens among persons with HIV/AIDS in Baltimore, Maryland," American Journal of Public Health, vol. 99, no. 6, pp. 1116-1122, 2009.

[231] H. Nahrevanian and M. Assmar, "Cryptosporidiosis in immunocompromised patients in the Islamic Republic of Iran," Journal of Microbiology, Immunology and Infection, vol. 41, no. 1, pp. 74-77, 2008.

[232] M. A. Blanco, A. Iborra, A. Vargas, E. Nsie, L. Mbá, and I. Fuentes, "Molecular characterization of Cryptosporidium isolates from humans in Equatorial Guinea," Transactions of the Royal Society of Tropical Medicine and Hygiene, vol. 103, no. 12, pp. 1282-1284, 2009. 
[233] S. S. Kumar, S. Ananthan, and P. Lakshmi, "Intestinal parasitic infection in HIV infected patients with diarrhoea in Chennai," Indian Journal of Medical Microbiology, vol. 20, no. 2, pp. 88-91, 2002.

[234] A. Curry and H. V. Smith, "Emerging pathogens: Isospora, Cyclospora and microsporidia," Parasitology, vol. 117, pp. S143-S159, 1998.

[235] S. S. R. Ajjampur, P. Sankaran, and G. Kang, "Cryptosporidium species in HIV-infected individuals in India: an overview," The National Medical Journal of India, vol. 21, no. 4, pp. 178-184, 2008.

[236] S. S. R. Ajjampur, P. Rajendran, S. Ramani et al., "Closing the diarrhoea diagnostic gap in Indian children by the application of molecular techniques," Journal of Medical Microbiology, vol. 57, no. 11, pp. 1364-1368, 2008.

[237] Y. A. L. Lim, R. A. Ahmad, and H. V. Smith, "Current status and future trends in Cryptosporidium and Giardia epidemiology in Malaysia," Journal of Water and Health, vol. 6, no. 2, pp. 239-254, 2008.

[238] A. Kurniawan, T. Karyadi, S. W. Dwintasari et al., "Intestinal parasitic infections in HIV/AIDS patients presenting with diarrhoea in Jakarta, Indonesia," Transactions of the Royal Society of Tropical Medicine and Hygiene, vol. 103, no. 9, pp. 892-898, 2009.

[239] C. Nuchjangreed, K. Boonrod, J. Ongerth, and P. Karanis, "Prevalence and molecular characterization of human and bovine Cryptosporidium isolates in Thailand," Parasitology Research, vol. 103, no. 6, pp. 1347-1353, 2008.

[240] V. A. Cama, C. Bern, I. M. Sulaiman et al., "Cryptosporidium species and genotypes in HIV-positive patients in Lima, Peru," The Journal of Eukaryotic Microbiology, vol. 50, pp. 531-533, 2003.

[241] M.-M. Deschamps, D. W. Fitzgerald, J. W. Pape, and W. D. Johnson Jr., "HIV infection in Haiti: natural history and disease progression," AIDS, vol. 14, no. 16, pp. 2515-2521, 2000.

[242] M. Kosek, C. Bern, and R. L. Guerrant, "The global burden of diarrhoeal disease, as estimated from studies published between 1992 and 2000," Bulletin of the World Health Organization, vol. 81, no. 3, pp. 197-204, 2003.

[243] N. Thapar and I. R. Sanderson, "Diarrhoea in children: an interface between developing and developed countries," The Lancet, vol. 363, no. 9409, pp. 641-653, 2004.

[244] P. Crook, R. Mayon-White, and M. Reacher, "Enhancing surveillance of cryptosporidiosis: test all faecal specimens from children," Communicable Disease and Public Health, vol. 5, no. 2, pp. 112-113, 2002.

[245] Communicable Diseases Branch, http://www.health.qld.gov $. \mathrm{au} / \mathrm{ph} / \mathrm{cdb} /$ default.asp.

[246] N. Coetzee, O. Edeghere, J. Orendi, R. Chalmers, and L. Morgan, "A swimming pool-associated outbreak of cryptosporidiosis in Staffordshire, England, October to December 2007," Eurosurveillance, vol. 13, no. 45, Article ID 19028, 2008.

[247] C. McGuigan, "Cryptosporidium outbreak after a visit to a wildlife centre in northeast Scotland: 62 confirmed cases," Eurosurveillance, vol. 10, no. 4, Article ID 2691, 2005.

[248] M. Jones, D. Boccia, M. Kealy et al., "Cryptosporidium outbreak linked to interactive water feature, UK: importance of guidelines," Eurosurveillance, vol. 11, no. 4, pp. 126-128, 2006.

[249] "Surveillance of waterborne disease and water quality: July to December 2003," Communicable Disease Report Weekly, vol. 14, no. 15, 2004.
[250] J. G. Mattsson, M. Insulander, M. Lebbad, C. Björkman, and B. Svenungsson, "Molecular typing of Cryptosporidium parvum associated with a diarrhoea outbreak identifies two sources of exposure," Epidemiology and Infection, vol. 136, no. 8, pp. 1147-1152, 2008.

[251] M. R. Hoek, I. Oliver, M. Barlow, L. Heard, R. Chalmers, and S. Paynter, "Outbreak of Cryptosporidium parvum among children after a school excursion to an adventure farm, South West England," Journal of Water and Health, vol. 6, no. 3, pp. 333-338, 2008.

[252] M. T. Ortega, A. Vergara, J. Guimbao, A. Clavel, P. Gavín, and A. Ruiz, "Brote de diarrea y transmisión de Cryptosporidium hominis asociados al uso de pañal en niños," Medicina Clinica, vol. 127, no. 17, pp. 653-656, 2006.

[253] P. E. Rodriguez-Salinas, A. J. Aragon Pena, T. M. Allue, M. A. Lopez Perez, M. M. Jimenez, and M. Dominiguez Rodriguez, "Outbreak of cryptosporidiosis in Guadarrama (Autonomous Community of Madrid)," Revista Española de Salud Pública, vol. 74, no. 5-6, pp. 527-536, 2000.

[254] A. Bajer, M. Bednarska, S. M. Cacciò et al., "Genotyping of Cryptosporidium isolates from human clinical cases in Poland," Parasitology Research, vol. 103, no. 1, pp. 37-42, 2008.

[255] M. V. Lavdovskaia, A. I. Lysenko, I. P. Gorbunova et al., "The characteristics of cryptosporidiosis epidemiology in the European part of Russia," Meditsinskaia parazitologiia $i$ parazitarnye bolezni, no. 3, pp. 8-11, 1996.

[256] A. Caprioli, C. Pezzella, R. Morelli et al., "Enteropathogens associated with childhood diarrhea in Italy. The Italian Study Group on Gastrointestinal Infections," Pediatric Infectious Disease Journal, vol. 15, no. 10, pp. 876-883, 1996.

[257] O. Brandonisio, A. Marangi, M. A. Panaro et al., "Prevalence of Cryptosporidium in children with enteritis in Southern Italy," European Journal of Epidemiology, vol. 12, no. 2, pp. 187-190, 1996.

[258] R. Jue, T. Schmalz, K. Carter, and R. J. Nett, "Outbreak of cryptosporidiosis associated with a splash park-Idaho, 2007," Morbidity and Mortality Weekly Report, vol. 58, no. 22, pp. 615-618, 2009.

[259] L. M. Causer, T. Handzel, P. Welch et al., "An outbreak of Cryptosporidium hominis infection at an Illinois recreational waterpark," Epidemiology and Infection, vol. 134, no. 1, pp. 147-156, 2006.

[260] P. Minshew, K. Ward, Z. Mulla, et al., “Outbreak of gastroenteritis associated with an interactive water fountain at a beachside park, Florida, 1999," Morbidity and Mortality Weekly Report, vol. 49, no. 25, pp. 565-568, 2000.

[261] L. Eisenstein, D. Bodager, and D. Ginzl, "Outbreak of giardiasis and cryptosporidiosis associated with a neighborhood interactive water fountain-Florida, 2006," Journal of Environmental Health, vol. 71, no. 3, pp. 18-22, 2008.

[262] C. M. Harper, N. A. Cowell, B. C. Adams, A. J. Langley, and T. D. Wohlsen, "Outbreak of Cryptosporidium linked to drinking unpasteurised milk," Communicable Diseases Intelligence, vol. 26, no. 3, pp. 449-450, 2002.

[263] R. Gelletlie, J. Stuart, N. Soltanpoor, R. Armstrong, and G. Nichols, "Cryptosporidiosis associated with school milk," The Lancet, vol. 350, no. 9083, pp. 1005-1006, 1997.

[264] N. Stefanogiannis, M. McLean, and H. Van Mil, "Outbreak of cryptosporidiosis linked with a farm event," The New Zealand Medical Journal, vol. 114, no. 1144, pp. 519-521, 2001. 
[265] J. E. Aledort, A. Ronald, S. M. Le Blancq, et al., "Reducing the burden of HIV/AIDS in infants: the contribution of improved diagnostics," Nature, vol. 444, supplement 1, pp. 19-28, 2006.

[266] K. A. Ricci, F. Girosi, P. I. Tarr, et al., "Reducing stunting among children: the potential contribution of diagnostics," Nature, vol. 4444, supplement 1, pp. 29-38, 2006.

[267] S. M. Mor, J. K. Tumwine, E. N. Naumova, G. Ndeezi, and S. Tzipori, "Microsporidiosis and malnutrition in children with persistent diarrhea, Uganda," Emerging Infectious Diseases, vol. 15, no. 1, pp. 49-52, 2009.

[268] L. Pelayo, F. A. Nuñez, L. Rojas et al., "Molecular and epidemiological investigations of cryptosporidiosis in Cuban children," Annals of Tropical Medicine and Parasitology, vol. 102, no. 8, pp. 659-669, 2008.

[269] S. M. Mor and S. Tzipori, "Cryptosporidiosis in children in sub-Saharan Africa: a lingering challenge," Clinical Infectious Diseases, vol. 47, no. 7, pp. 915-921, 2008.

[270] D. Ayalew, E. Boelee, T. Endeshaw, and B. Petros, "Cryptosporidium and Giardia infection and drinking water sources among children in Lege Dini, Ethiopia," Tropical Medicine \& International Health, vol. 13, no. 4, pp. 472-475, 2008.

[271] M. S. Dlamini, S. J. Nkambule, and A. M. Grimason, "First report of cryptosporidiosis in paediatric patients in Swaziland," International Journal of Environmental Health Research, vol. 15, no. 5, pp. 393-396, 2005.

[272] F. Gay-Andrieu, E. Adehossi, H. Illa, A. Garba Ben, H. Kourna, and H. Boureima, "Prevalence of cryptosporidiosis in Niamey (Niger) pediatric hospital patients," Bulletin de la Société de Pathologie Exotique, vol. 100, no. 3, pp. 193-196, 2007.

[273] NetEpi, http://code.google.com/p/netepi.

[274] Epi Info software, http://www.who.int/chp/steps/resources/ EpiInfo/en/index.html.

[275] M. Areeshi, W. Dove, D. Papaventsis et al., "Cryptosporidium species causing acute diarrhoea in children in Antananarivo, Madagascar," Annals of Tropical Medicine and Parasitology, vol. 102, no. 4, pp. 309-315, 2008.

[276] U. Aksoy, C. Akisu, S. Sahin et al., "First reported waterborne outbreak of cryptosporidiosis with Cyclospora co-infection in Turkey," Eurosurveillance, vol. 12, no. 2, Article ID 3142, 2007.

[277] L. J. Robertson, T. Forberg, L. Hermansen, B. K. Gjerde, J. O. Alvsvåg, and N. Langeland, "Cryptosporidium parvum infections in Bergen, Norway, during an extensive outbreak of waterborne giardiasis in autumn and winter 2004," Applied and Environmental Microbiology, vol. 72, no. 3, pp. 22182220, 2006.

[278] D. E. Katz and D. N. Taylor, "Parasitic infections of the gastrointestinal tract," Gastroenterology Clinics of North America, vol. 30, no. 3, pp. 797-815, 2001.

[279] R. Haque, D. Mondal, B. D. Kirkpatrick et al., "Epidemiologic and clinical characteristics of acute diarrhea with emphasis on Entamoeba histolytica infections in preschool children in an urban slum of Dhaka, Bangladesh," The American Journal of Tropical Medicine and Hygiene, vol. 69, no. 4, pp. 398-405, 2003.

[280] D. Mondal, R. Haque, R. B. Sack, B. D. Kirkpatrick, and W. A. Petri Jr., "Attribution of malnutrition to causespecific diarrheal illness: evidence from a prospective study of preschool children in Mirpur, Dhaka, Bangladesh," The American Journal of Tropical Medicine and Hygiene, vol. 80, no. 5, pp. 824-826, 2009.
[281] B. Wongstitwilairoong, A. Srijan, O. Serichantalergs et al., "Intestinal parasitic infections among pre-school children in Sangkhlaburi, Thailand," The American Journal of Tropical Medicine and Hygiene, vol. 76, no. 2, pp. 345-350, 2007.

[282] J. M. Easow, C. Mukhopadhyay, G. Wilson, S. Guha, B. Y. Jalan, and P. G. Shivananda, "Emerging opportunistic protozoa and intestinal pathogenic protozoal infestation profile in children of western Nepal," Nepal Medical College Journal, vol. 7, no. 2, pp. 134-137, 2005.

[283] G. Börekçı and A. Uzel, "The determination of intestinal parasites, physical growth and hygiene behaviors of children in the Mersin City Social Service Child Care Centre," Türkiye Parazitolojii Derisi, vol. 33, no. 1, pp. 63-72, 2009.

[284] M. Matsubayashi, K. Takami, I. Kimata et al., "Survey of Cryptosporidium spp. and Giardia spp. infections in various animals at a zoo in Japan," Journal of Zoo and Wildlife Medicine, vol. 36, no. 2, pp. 331-335, 2005.

[285] C. Blackmore, J. R. Dunn, K. E. Smith, et al., "Compendium of measures to prevent disease associated with animals in public settings, 2009: National Association of State Public Health Veterinarians, Inc. (NASPHV)," Morbidity and Mortality Weekly Report, vol. 58, no. RR-5, pp. 1-21, 2009.

[286] R. P. Smith, R. M. Chalmers, K. Elwin et al., "Investigation of the role of companion animals in the zoonotic transmission of cryptosporidiosis," Zoonoses and Public Health, vol. 56, no. 1, pp. 24-33, 2009.

[287] G. J. Leitch and Q. He, "Putative anticryptosporidial agents tested with an immunodeficient mouse model," Antimicrobial Agents and Chemotherapy, vol. 38, no. 4, pp. 865-867, 1994.

[288] V. Cama, R. H. Gilman, A. Vivar et al., "Mixed Cryptosporidium infections and HIV," Emerging Infectious Diseases, vol. 12, no. 6, pp. 1025-1028, 2006.

[289] E. B. Steinberg, C. E. Mendoza, R. Glass et al., "Prevalence of infection with waterborne pathogens: a seroepidemiologic study in children 6-36 months old in San Juan Sacatepequez, Guatemala," The American Journal of Tropical Medicine and Hygiene, vol. 70, no. 1, pp. 83-88, 2004.

[290] F. A. Núñez, O. M. González, I. González, A. A. Escobedo, and R. A. Cordoví, "Intestinal coccidia in Cuban pediatric patients with diarrhea," Memórias do Instituto Oswaldo Cruz, vol. 98, no. 4, pp. 539-542, 2003.

[291] D. Carnicer-Pont, R. Atenstaedt, M. Walker et al., "An outbreak of cryptosporidiosis in Wales, November 2005," Eurosurveillance, vol. 10, no. 12, Article ID 2854, 2005.

[292] A. Galmes, A. Nicolau, G. Arbona, A. Smith-Palmer, G. Hernandez Pezzi, and P. Soler, "Cryptosporidiosis outbreak in British tourists who stayed at a hotel in Majorca, Spain," Eurosurveillance, vol. 7, no. 33, Article ID 2275, 2003.

[293] "Surveillance of waterborne disease and water quality: January to June 2003, and summary of 2002," Communicable Disease Report Weekly, vol. 13, no. 41, 2003.

[294] "Surveillance of waterborne disease and water quality: January to June 2002, and summary of 2001," Communicable Disease Report Weekly, vol. 12, no. 37, 2002.

[295] "Surveillance of waterborne disease and water quality: January to June 2001, and summary of 2000," Communicable Disease Report Weekly, vol. 11, no. 45, 2001.

[296] R. Stirling, J. Aramini, A. Ellis et al., "Waterborne cryptosporidiosis outbreak, North Battleford, Saskatchewan, Spring 2001," Canada Communicable Disease Report, vol. 27, no. 22, pp. 185-192, 2001. 
[297] J. Macey, L. Lior, A. Johnston et al., "Outbreak of diarrheal illness in attendees at a Ukrainian dance festival, Dauphin, Manitoba-May 2001," Canada Communicable Disease Report, vol. 28, no. 17, pp. 141-145, 2002.

[298] A. Hajdu, L. Vold, T. A. Østmo et al., "Investigation of Swedish cases reveals an outbreak of cryptosporidiosis at a Norwegian hotel with possible links to in-house water systems," BMC Infectious Diseases, vol. 8, article 152, 2008.

[299] R. Gait, R. H. Soutar, M. Hanson, C. Fraser, and R. Chalmers, "Outbreak of cryptosporidiosis among veterinary students," The Veterinary Record, vol. 162, no. 26, pp. 843-845, 2008.

[300] S. O. Brockmann, C. Dreweck, C. Wagner-Wiening et al., "Serological and epidemiological analysis of an outbreak of gastroenteritis among military recruits in Germany caused by Cryptosporidium parvum," Infection, vol. 36, no. 5, pp. 450457, 2008.

[301] H. Yoshida, M. Matsuo, T. Miyoshi et al., "An outbreak of cryptosporidiosis suspected to be related to contaminated food, October 2006, Sakai City, Japan," Japanese Journal of Infectious Diseases, vol. 60, no. 6, pp. 405-407, 2007.

[302] N. Pandak, K. Zeljka, and A. Cvitkovic, "A family outbreak of cryptosporidiosis: probable nosocomial infection and person-to-person transmission," Wiener Klinische Wochenschrift, vol. 118, no. 15-16, pp. 485-487, 2006.

[303] K. M. Kiang, J. M. Scheftel, F. T. Leano et al., "Recurrent outbreaks of cryptosporidiosis associated with calves among students at an educational farm programme, Minnesota, 2003," Epidemiology and Infection, vol. 134, no. 4, pp. 878886, 2006.

[304] G. Preiser, L. Preiser, and L. Madeo, "An outbreak of cryptosporidiosis among veterinary science students who work with calves," Journal of American College Health, vol. 51, no. 5, pp. 213-215, 2003.

[305] F. Dalle, P. Roz, G. Dautin et al., "Molecular characterization of isolates of waterborne Cryptosporidium spp. Collected during an outbreak of gastroenteritis in South Burgundy, France," Journal of Clinical Microbiology, vol. 41, no. 6, pp. 2690-2693, 2003.

[306] R. H. Ashbolt, D. J. Coleman, A. Misrachi, J. M. Conti, and M. D. Kirk, "An outbreak of cryptosporidiosis associated with an animal nursery at a regional fair," Communicable Diseases Intelligence, vol. 27, no. 2, pp. 244-249, 2003.

[307] E. M. D. N. Gonçalves, A. J. da Silva, M. B. D. P. Eduardo et al., "Multilocus genotyping of Cryptosporidium hominis associated with diarrhea outbreak in a day care unit in São Paulo," Clinics, vol. 61, no. 2, pp. 119-126, 2006.

[308] A. D. Howe, S. Forster, S. Morton et al., "Cryptosporidium oocysts in a water supply associated with a cryptosporidiosis outbreak," Emerging Infectious Diseases, vol. 8, no. 6, pp. 619624, 2002.

[309] P. R. Hunter, D. C. Wilkinson, I. R. Lake et al., "Microsatellite typing of Cryptosporidium parvum in isolates from a waterborne outbreak," Journal of Clinical Microbiology, vol. 46, no. 11, pp. 3866-3867, 2008.

[310] A. Egorov, F. Frost, T. Muller, E. Naumova, A. Tereschenko, and T. Ford, "Serological evidence of Cryptosporidium infections in a Russian city and evaluation of risk factors for infections," Annals of Epidemiology, vol. 14, no. 2, pp. 129136, 2004.

[311] E. S. Quiroz, C. Bern, J. R. MacArthur et al., "An outbreak of cryptosporidiosis linked to a foodhandler," The Journal of Infectious Diseases, vol. 181, no. 2, pp. 695-700, 2000. 


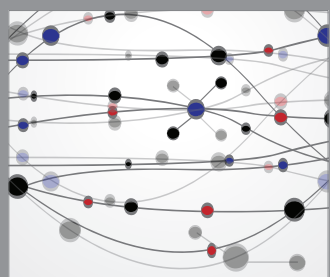

The Scientific World Journal
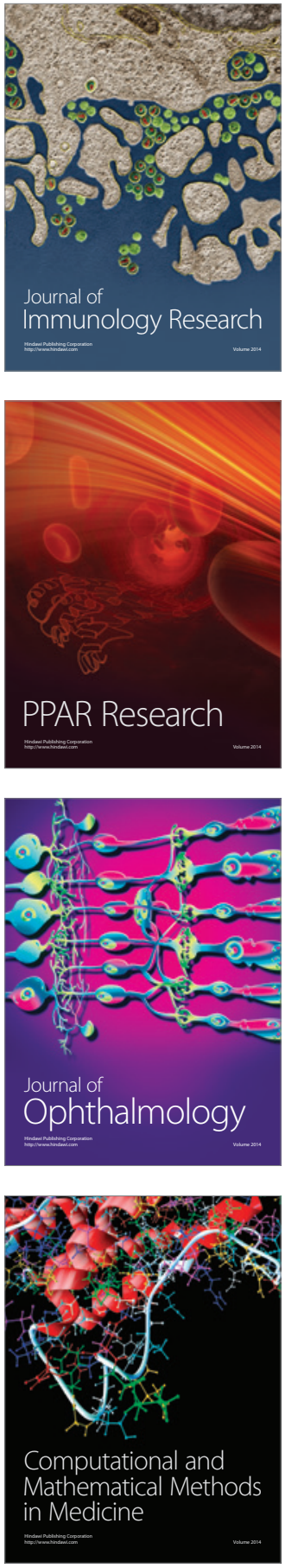

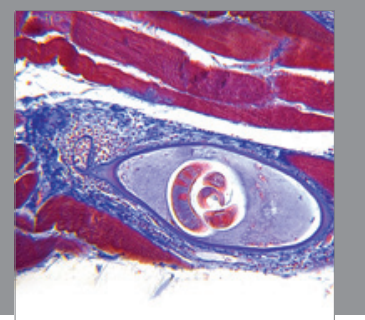

Gastroenterology

Research and Practice
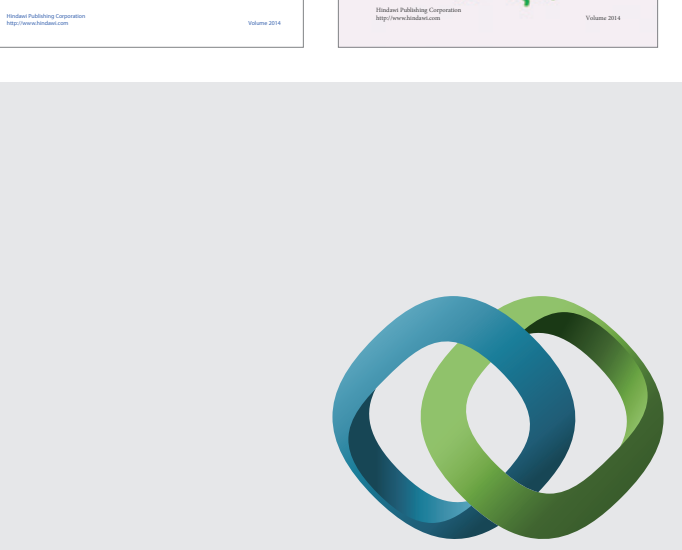

\section{Hindawi}

Submit your manuscripts at

http://www.hindawi.com
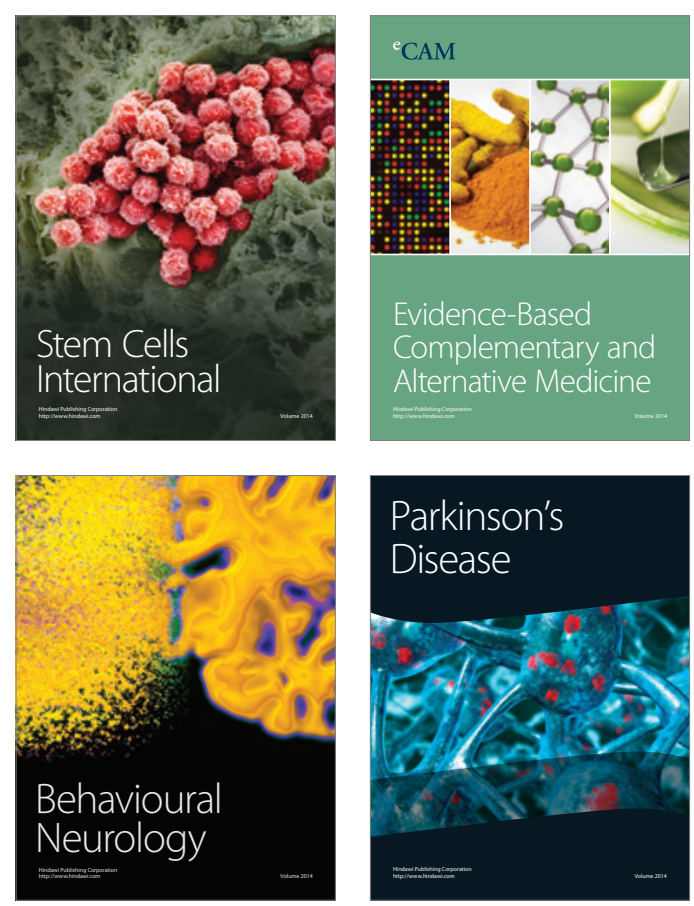

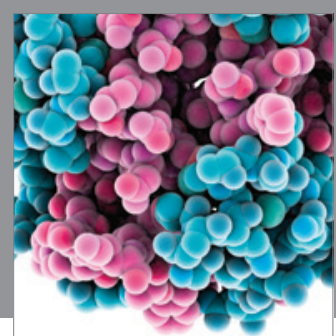

Journal of
Diabetes Research

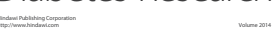

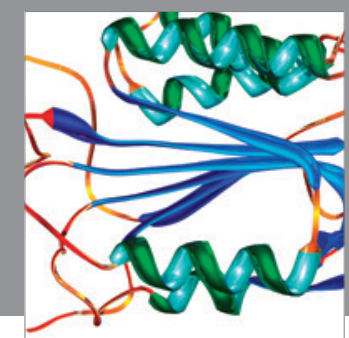

Disease Markers
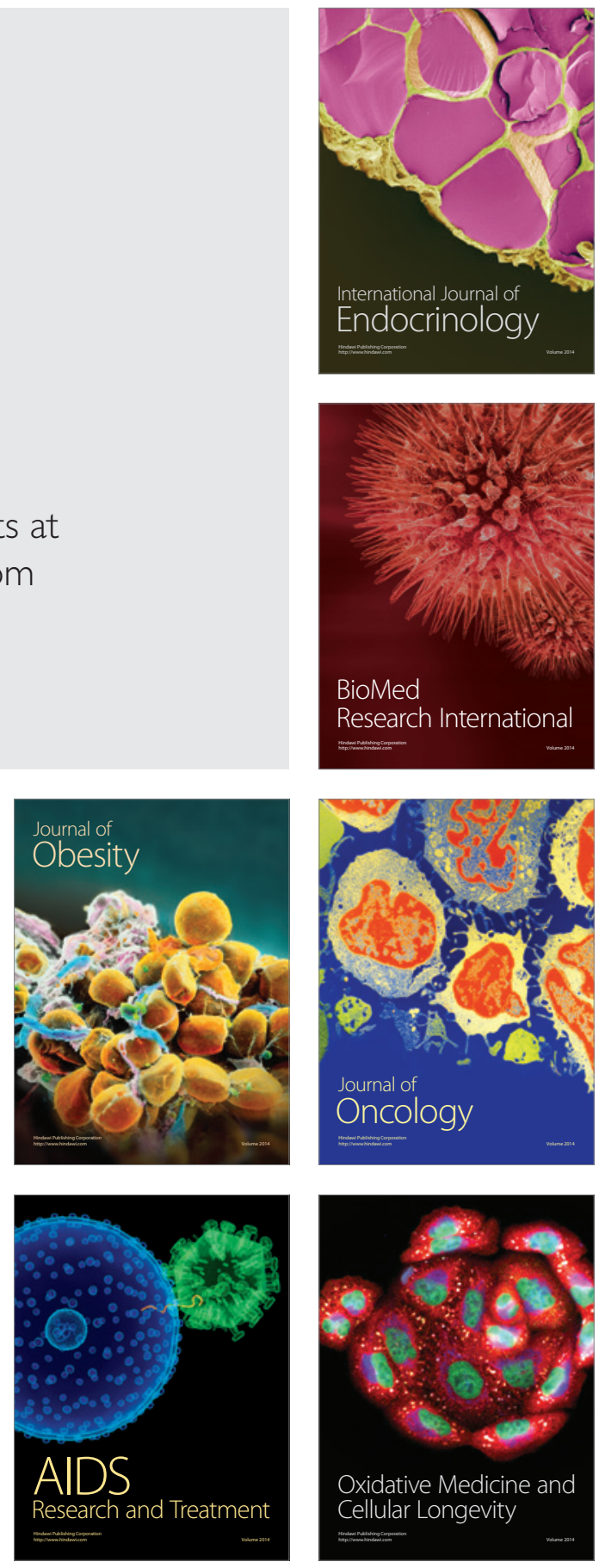